Research Article

\title{
Growth of Accreting Supermassive Black Hole Seeds and Neutrino Radiation
}

\author{
Gagik Ter-Kazarian \\ Division of Theoretical Astrophysics, Ambartsumian Byurakan Astrophysical Observatory, Byurakan, 378433 Aragatsotn, Armenia
}

Correspondence should be addressed to Gagik Ter-Kazarian; gago_50@yahoo.com

Received 1 May 2014; Revised 26 June 2014; Accepted 27 June 2014

Academic Editor: Gary Wegner

Copyright (C) 2015 Gagik Ter-Kazarian. This is an open access article distributed under the Creative Commons Attribution License, which permits unrestricted use, distribution, and reproduction in any medium, provided the original work is properly cited.

In the framework of microscopic theory of black hole (MTBH), which explores the most important processes of rearrangement of vacuum state and spontaneous breaking of gravitation gauge symmetry at huge energies, we have undertaken a large series of numerical simulations with the goal to trace an evolution of the mass assembly history of 377 plausible accreting supermassive black hole seeds in active galactic nuclei (AGNs) to the present time and examine the observable signatures today. Given the redshifts, masses, and luminosities of these black holes at present time collected from the literature, we compute the initial redshifts and masses of the corresponding seed black holes. For the present masses $M_{\mathrm{BH}} / M_{\odot} \simeq 1.1 \times 10^{6}$ to $1.3 \times 10^{10}$ of 377 black holes, the computed intermediate seed masses are ranging from $M_{\mathrm{BH}}^{\text {Seed }} / M_{\odot} \simeq 26.4$ to $2.9 \times 10^{5}$. We also compute the fluxes of ultrahigh energy (UHE) neutrinos produced via simple or modified URCA processes in superdense protomatter nuclei. The AGNs are favored as promising pure UHE neutrino sources, because the computed neutrino fluxes are highly beamed along the plane of accretion disk, peaked at high energies, and collimated in smaller opening angle $(\theta \ll 1)$.

\section{Introduction}

With typical bolometric luminosities $\sim 10^{45-48} \mathrm{erg} \mathrm{s}^{-1}$, the AGNs are amongst the most luminous emitters in the universe, particularly at high energies (gamma-rays) and radio wavelengths. From its historical development, up to current interests, the efforts in the AGN physics have evoked the study of a major unsolved problem of how efficiently such huge energies observed can be generated. This energy scale severely challenges conventional source models. The huge energy release from compact regions of AGN requires extremely high efficiency (typically $\geq 10$ per cent) of conversion of rest mass to other forms of energy. This serves as the main argument in favour of supermassive black holes, with masses of millions to billions of times the mass of the Sun, as central engines of massive AGNs. The astrophysical black holes come in a wide range of masses, from $\geq 3 M_{\odot}$ for stellar mass black holes [1] to $\sim 10^{10} M_{\odot}$ for supermassive black holes $[2,3]$. Demography of local galaxies suggests that most galaxies harbour quiescent supermassive black holes in their nuclei at the present time and that the mass of the hosted black hole is correlated with properties of the host bulge. The visible universe should therefore contain at least 100 billion supermassive black holes. A complex study of evolution of AGNs requires an answer to the key questions such as how did the first black holes form, how did massive black holes get to the galaxy centers, and how did they grow in accreting mass, namely, an understanding of the important phenomenon of mass assembly history of accreting supermassive black hole seeds. The observations support the idea that black holes grow in tandem with their hosts throughout cosmic history, starting from the earliest times. While the exact mechanism for the formation of the first black holes is not currently known, there are several prevailing theories [4]. However, each proposal towards formation and growth of initial seed black holes has its own advantage and limitations in proving the whole view of the issue. In this report we review the mass assembly history of 377 plausible accreting supermassive black hole seeds in AGNs and their neutrino radiation in the framework of gravitation theory, which explores the most important processes of rearrangement of vacuum state and a spontaneous breaking of gravitation gauge symmetry at huge energies. We will proceed according to the following structure. Most observational, theoretical, and computational 
aspects of the growth of black hole seeds are summarized in Section 2. The other important phenomenon of ultrahigh energy cosmic rays, in relevance to AGNs, is discussed in Section 3. The objectives of suggested approach are outlined in Section 4. In Section 5 we review the spherical accretion on superdense protomatter nuclei, in use. In Section 6 we discuss the growth of the seed black hole at accretion and derive its intermediate mass, initial redshift, and neutrino preradiation time (PRT). Section 7 is devoted to the neutrino radiation produced in superdense protomatter nuclei. The simulation results of the seed black hole intermediate masses, PRTs, seed redshifts, and neutrino fluxes for 377 AGN black holes are brought in Section 8. The concluding remarks are given in Section 9. We will refrain from providing lengthy details of the proposed gravitation theory at huge energies and neutrino flux computations. For these the reader is invited to visit the original papers and appendices of the present paper. In the latter we also complete the spacetime deformation theory, in the model context of gravitation, by new investigation of building up the complex of distortion (DC) of spacetime continuum and showing how it restores the world-deformation tensor, which still has been put in by hand. Finally, note that we regard the considered black holes only as the potential neutrino sources. The obtained results, however, may suffer if not all live black holes at present reside in final stage of their growth driven by the formation of protomatter disk at accretion and they radiate neutrino. We often suppress the indices without notice. Unless otherwise stated, we take geometrized units throughout this paper.

\section{A Breakthrough in Observational and Computational Aspects on Growth of Black Hole Seeds}

Significant progress has been made in the last few years in understanding how supermassive black holes form and grow. Given the current masses of $10^{6-9} M_{\odot}$, most black hole growth happens in the AGN phase. A significant fraction of the total black hole growth, 60\% [6], happens in the most luminous AGN, quasars. In an AGN phase, which lasts $\sim 10^{8}$ years, the central supermassive black hole can gain up to $\sim 10^{7-8} M_{\odot}$, so even the most massive galaxies will have only a few of these events over their lifetime. Aforesaid gathers support especially from a breakthrough made in recent observational, theoretical, and computational efforts in understanding of evolution of black holes and their host galaxies, particularly through self-regulated growth and feedback from accretionpowered outflows; see, for example, [4, 7-18]. Whereas the multiwavelength methods are used to trace the growth of seed BHs, the prospects for future observations are reviewed. The observations provide strong support for the existence of a correlation between supermassive black holes and their hosts out to the highest redshifts. The observations of the quasar luminosity function show that the most supermassive black holes get most of their mass at high redshift, while at low redshift only low mass black holes are still growing [19]. This is observed in both optical [20] and hard Xray luminosity functions $[19,21]$, which indicates that this result is independent of obscuration. Natarajan [13] has reported that the initial black hole seeds form at extremely high redshifts from the direct collapse of pregalactic gas discs. Populating dark matter halos with seeds formed in this fashion and using a Monte-Carlo merger tree approach, he has predicted the black hole mass function at high redshifts and at the present time. The most aspects of the models that describe the growth and accretion history of supermassive black holes and evolution of this scenario have been presented in detail by $[9,10]$. In these models, at early times the properties of the assembling black hole seeds are more tightly coupled to properties of the dark matter halo as their growth is driven by the merger history of halos. While a clear picture of the history of black hole growth is emerging, significant uncertainties still remain [14], and in spite of recent advances $[6,13]$, the origin of the seed black holes remains an unsolved problem at present. The NuSTAR deep high-energy observations will enable obtaining a nearly complete AGN survey, including heavily obscured Comptonthick sources, up to $z \sim 1.5$ [22]. A similar mission, ASTRO$\mathrm{H}$ [23], will be launched by Japan in 2014. These observations in combination with observations at longer wavelengths will allow for the detection and identification of most growing supermassive black holes at $z \sim 1$. The ultradeep X-ray and near-infrared surveys covering at least $\sim 1 \mathrm{deg}^{2}$ are required to constrain the formation of the first black hole seeds. This will likely require the use of the next generation of spacebased observatories such as the James Webb Space Telescope and the International X-ray Observatory. The superb spatial resolution and sensitivity of the Atacama Large Millimeter Array (ALMA) [24] will revolutionize our understanding of galaxy evolution. Combining these new data with existing multiwavelength information will finally allow astrophysicists to pave the way for later efforts by pioneering some of the census of supermassive black hole growth, in use today.

\section{UHE Cosmic-Ray Particles}

The galactic sources like supernova remnants (SNRs) or microquasars are thought to accelerate particles at least up to energies of $3 \times 10^{15} \mathrm{eV}$. The ultrahigh energy cosmicray (UHECR) particles with even higher energies have since been detected (comprehensive reviews can be found in [2529]). The accelerated protons or heavier nuclei up to energies exceeding $10^{20} \mathrm{eV}$ are firstly observed by [30]. The cosmicray events with the highest energies so far detected have energies of $2 \times 10^{11} \mathrm{GeV}$ [31] and $3 \times 10^{11} \mathrm{GeV}$ [32]. These energies are $10^{7}$ times higher than the most energetic manmade accelerator, the LHC at CERN. These highest energies are believed to be reached in extragalactic sources like AGNs or gamma-ray bursts (GRBs). During propagation of such energetic particles through the universe, the threshold for pion photoproduction on the microwave background is $\sim 2$ $\times 10^{10} \mathrm{GeV}$, and at $\sim 3 \times 10^{11} \mathrm{GeV}$ the energy-loss distance is about $20 \mathrm{Mpc}$. Propagation of cosmic rays over substantially larger distances gives rise to a cutoff in the spectrum at $~$ $10^{11} \mathrm{GeV}$ as was first shown by $[33,34]$, the GZK cutoff. The recent confirmation $[35,36]$ of GZK suppression in the 
cosmic-ray energy spectrum indicates that the cosmic rays with energies above the GZK cutoff, $E_{\mathrm{GZK}} \sim 40 \mathrm{EeV}$, mostly come from relatively close (within the GZK radius, $r_{\mathrm{GZK}} \sim$ $100 \mathrm{Mpc}$ ) extragalactic sources. However, despite the detailed measurements of the cosmic-ray spectrum, the identification of the sources of the cosmic-ray particles is still an open question as they are deflected in the galactic and extragalactic magnetic fields and hence have lost all information about their origin when reaching Earth. Only at the highest energies beyond $\sim 10^{19.6} \mathrm{GeV}$ cosmic-ray particles may retain enough directional information to locate their sources. The latter must be powerful enough to sustain the energy density in extragalactic cosmic rays of about $3 \times 10^{-19} \mathrm{erg} \mathrm{cm}^{-3}$ which is equivalent to $\sim 8 \times 10^{44} \mathrm{erg} \mathrm{Mpc}^{-3} \mathrm{yr}^{-1}$. Though it has not been possible up to now to identify the sources of galactic or extragalactic cosmic rays, general considerations allow limiting potential source classes. For example, the existing data on the cosmic-ray spectrum and on the isotropic $100 \mathrm{MeV}$ gammaray background limit significantly the parameter space in which topological defects can generate the flux of the highest energy cosmic rays and rule out models with the standard $\mathrm{X}$-particle mass of $10^{16} \mathrm{GeV}$ and higher [37]. Eventually, the neutrinos will serve as unique astronomical messengers, and they will significantly enhance and extend our knowledge on galactic and extragalactic sources of the UHE universe. Indeed, except for oscillations induced by transit in a vacuum Higgs field, neutrinos can penetrate cosmological distances and their trajectories are not deflected by magnetic fields as they are neutral, providing powerful probes of high energy astrophysics in ways which no other particle can. Moreover, the flavor composition of neutrinos originating at astrophysical sources can serve as a probe of new physics in the electroweak sector. Therefore, an appealing possibility among the various hypotheses of the origin of UHECR is so-called Z-burst scenario [38-51]. This suggests that if $\mathrm{ZeV}$ astrophysical neutrino beam is sufficiently strong, it can produce a large fraction of observed UHECR particles within $100 \mathrm{Mpc}$ by hitting local light relic neutrinos clustered in dark halos and form UHECR through the hadronic Z (s-channel production) and $\mathrm{W}$-bosons ( $\mathrm{t}$-channel production) decays by weak interactions. The discovery of UHE neutrino sources would also clarify the production mechanism of the GeV-TeV gamma rays observed on Earth $[43,52,53]$ as $\mathrm{TeV}$ photons are also produced in the up-scattering of photons in reactions to accelerated electrons (inverse-Compton scattering). The direct link between $\mathrm{TeV}$ gamma-ray photons and neutrinos through the charged and neutral pion production, which is well known from particle physics, allows for a quite robust prediction of the expected neutrino fluxes provided that the sources are transparent and the observed gamma rays originate from pion decay. The weakest link in the Z-burst hypothesis is probably both unknown boosting mechanism of the primary neutrinos up to huge energies of hundreds $\mathrm{ZeV}$ and their large flux required at the resonant energy $E_{v} \simeq$ $M_{Z}^{2} /\left(2 m_{\nu}\right) \simeq 4.2 \times 10^{21} \mathrm{eV}\left(\mathrm{eV} / m_{\nu}\right)$ well above the GZK cutoff. Such a flux severely challenges conventional source models. Any concomitant photon flux should not violate existing upper limits $[37,48,49,54]$. The obvious question is then raised: where in the Cosmos are these neutrinos coming from? It turns out that currently, at energies in excess of $10^{19} \mathrm{eV}$, there are only two good candidate source classes for UHE neutrinos: AGNs and GRBs. The AGNs as significant point sources of neutrinos were analyzed in $[50,55,56]$. While hard to detect, neutrinos have the advantage of representing aforesaid unique fingerprints of hadron interactions and, therefore, of the sources of cosmic rays. Two basic event topologies can be distinguished: track-like patterns of detected Cherenkov light (hits) which originate from muons produced in charged-current interactions of muon neutrinos (muon channel); spherical hit patterns which originate from the hadronic cascade at the vertex of neutrino interactions or the electromagnetic cascade of electrons from chargedcurrent interactions of electron neutrinos (cascade channel). If the charged-current interaction happens inside the detector or in case of charged-current tau-neutrino interactions, these two topologies overlap which complicates the reconstruction. At the relevant energies, the neutrino is approximately collinear with the muon and, hence, the muon channel is the prime channel for the search for point-like sources of cosmic neutrinos. On the other hand, cascades deposit all of their energy inside the detector and therefore allow for a much better energy reconstruction with a resolution of a few $10 \%$. Finally, numerous reports are available at present in literature on expected discovery potential and sensitivity of experiments to neutrino point-like sources. Currently operating high energy neutrino telescopes attempt to detect UHE neutrinos, such as ANTARES $[57,58]$ which is the most sensitive neutrino telescope in the Northern Hemisphere, IceCube $[35,59-64]$ which is worldwide largest and hence most sensitive neutrino telescope in the Southern Hemisphere, BAIKAL [65], as well as the CR extended experiments of The Telescope Array [66], Pierre Auger Observatory [67, 68], and JEM-EUSO mission [69]. The JEM-EUSO mission, which is planned to be launched by a H2B rocket around 20152016, is designed to explore the extremes in the universe and fundamental physics through the detection of the extreme energy $\left(E>10^{20} \mathrm{eV}\right)$ cosmic rays. The possible origins of the soon-to-be famous 28 IceCube neutrino-PeV events [59-61] are the first hint for astrophysical neutrino signal. Aartsen et al. have published an observation of two $\sim 1 \mathrm{PeV}$ neutrinos, with a $P$ value $2.8 \sigma$ beyond the hypothesis that these events were atmospherically generated [59]. The analysis revealed an additional 26 neutrino candidates depositing "electromagnetic equivalent energies" ranging from about $30 \mathrm{TeV}$ up to $250 \mathrm{TeV}$ [61]. New results were presented at the IceCube Particle Astrophysics Symposium (IPA 2013) [6264]. If cosmic neutrinos are primarily of extragalactic origin, then the $100 \mathrm{GeV}$ gamma ray flux observed by Fermi-LAT constrains the normalization at $\mathrm{PeV}$ energies at injection, which in turn demands a neutrino spectral index $\Gamma<2.1[70]$.

\section{MTBH, Revisited: Preliminaries}

For the benefit of the reader, a brief outline of the key ideas behind the microscopic theory of black hole, as a guiding principle, is given in this section to make the rest of the 
paper understandable. There is a general belief reinforced by statements in textbooks that, according to general relativity (GR), a long-standing standard phenomenological black hole model (PBHM) - namely, the most general Kerr-Newman black hole model, with parameters of mass $(M)$, angular momentum $(J)$, and charge $(Q)$, still has to be put in by hand-can describe the growth of accreting supermassive black hole seed. However, such beliefs are suspect and should be critically reexamined. The PBHM cannot be currently accepted as convincing model for addressing the aforementioned problems, because in this framework the very source of gravitational field of the black hole is a kind of curvature singularity at the center of the stationary black hole. A meaningless central singularity develops which is hidden behind the event horizon. The theory breaks down inside the event horizon which is causally disconnected from the exterior world. Either the Kruskal continuation of the Schwarzschild ( $J=0, Q=0)$ metric, or the $\operatorname{Kerr}(Q=0)$ metric, or the Reissner-Nordstrom $(J=0)$ metric, shows that the static observers fail to exist inside the horizon. Any object that collapses to form a black hole will go on to collapse to a singularity inside the black hole. Thereby any timelike worldline must strike the central singularity which wholly absorbs the infalling matter. Therefore, the ultimate fate of collapsing matter once it has crossed the black hole surface is unknown. This, in turn, disables any accumulation of matter in the central part and, thus, neither the growth of black holes nor the increase of their massenergy density could occur at accretion of outside matter, or by means of merger processes. As a consequence, the mass and angular momentum of black holes will not change over the lifetime of the universe. But how can one be sure that some hitherto unknown source of pressure does not become important at huge energies and halt the collapse? To fill the void which the standard PBHM presents, one plausible idea to innovate the solution to alluded key problems would appear to be the framework of microscopic theory of black hole. This theory has been originally proposed by [71] and references therein and thoroughly discussed in [72-75]. Here we recount some of the highlights of the MTBH, which is the extension of PBHM and rather completes it by exploring the most important processes of rearrangement of vacuum state and a spontaneous breaking of gravitation gauge symmetry at huge energies $[71,74,76]$. We will not be concerned with the actual details of this framework but only use it as a backdrop to validate the theory with some observational tests. For details, the interested reader is invited to consult the original papers. Discussed gravitational theory is consistent with GR up to the limit of neutron stars. But this theory manifests its virtues applied to the physics of internal structure of galactic nuclei. In the latter a significant change of properties of spacetime continuum, so-called inner distortion (ID), arises simultaneously with the strong gravity at huge energies (see Appendix A). Consequently the matter undergoes phase transition of second kind, which supplies a powerful pathway to form a stable superdense protomatter core (SPC) inside the event horizon. Due to this, the stable equilibrium holds in outward layers too and, thus, an accumulation of matter is allowed now around the SPC.
The black hole models presented in phenomenological and microscopic frameworks have been schematically plotted in Figure 1, to guide the eye. A crucial point of the MTBH is that a central singularity cannot occur, which is now replaced by finite though unbelievably extreme conditions held in the SPC, where the static observers existed. The SPC surrounded by the accretion disk presents the microscopic model of AGN. The SPC accommodates the highest energy scale up to hundreds of $\mathrm{ZeV}$ in central protomatter core which accounts for the spectral distribution of the resulting radiation of galactic nuclei. External physics of accretion onto the black hole in earlier part of its lifetime is identical to the processes in Schwarzschild's model. However, a strong difference in the model context between the phenomenological black hole and the SPC is arising in the second part of its lifetime (see Section 6). The seed black hole might grow up driven by the accretion of outside matter when it was getting most of its mass. An infalling matter with time forms the protomatter disk around the protomatter core tapering off faster at reaching out the thin edge of the event horizon. At this, metric singularity inevitably disappears (see appendices) and the neutrinos may escape through vista to outside world, even after the neutrino trapping. We study the growth of protomatter disk and derive the intermediate mass and initial redshift of seed black hole and examine luminosities, neutrino surfaces for the disk. In this framework, we have computed the fluxes of UHE neutrinos [75], produced in the medium of the SPC via simple (quark and pionic reactions) or modified URCA processes, even after the neutrino trapping (G. Gamow was inspired to name the process URCA after the name of a casino in Rio de Janeiro, when M. Schenberg remarked to him that "the energy disappears in the nucleus of the supernova as quickly as the money disappeared at that roulette table"). The "trapping" is due to the fact that as the neutrinos are formed in protomatter core at superhigh densities they experience greater difficulty escaping from the protomatter core before being dragged along with the matter; namely, the neutrinos are "trapped" comove with matter. The part of neutrinos annihilates to produce, further, the secondary particles of expected ultrahigh energies. In this model, of course, a key open question is to enlighten the mechanisms that trigger the activity, and how a large amount of matter can be steadily funneled to the central regions to fuel this activity. In high luminosity AGNs the large-scale internal gravitational instabilities drive gas towards the nucleus which trigger big starbursts, and the coeval compact cluster just formed. It seemed they have some connection to the nuclear fueling through mass loss of young stars as well as their tidal disruption and supernovae. Note that we regard the UHECR particles as a signature of existence of superdence protomatter sources in the universe. Since neutrino events are expected to be of sufficient intensity, our estimates can be used to guide investigations of neutrino detectors for the distant future.

\section{Spherical Accretion onto SPC}

As alluded to above, the MTBH framework supports the idea of accreting supermassive black holes which link to AGNs. In order to compute the mass accretion rate $\dot{M}$, in use, it is 


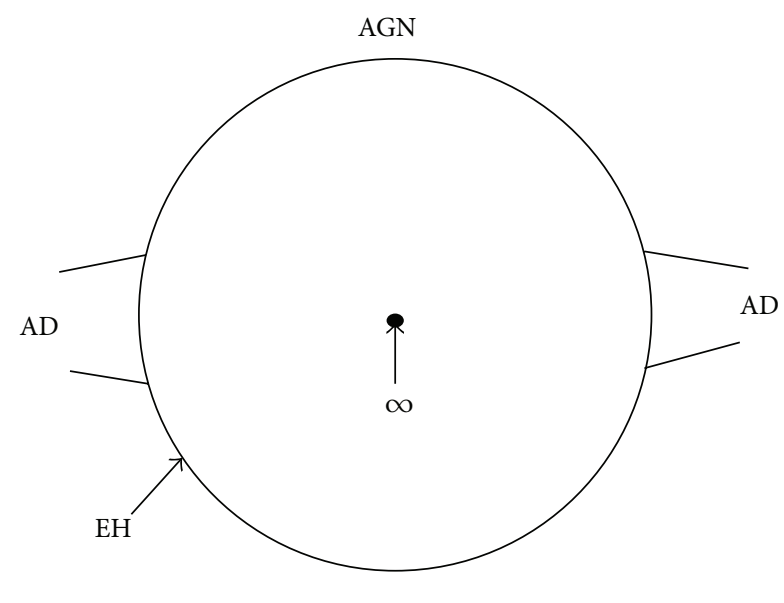

(a)

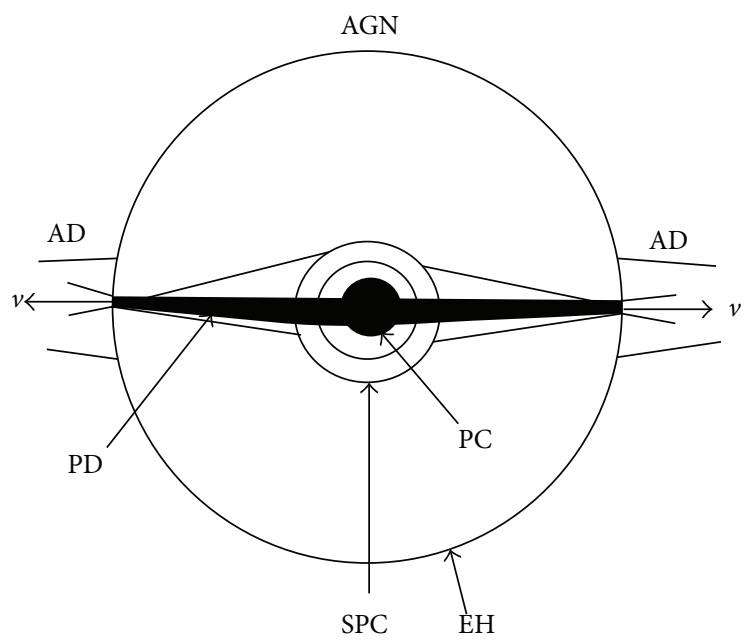

(b)

FIGURE 1: (a) The phenomenological model of AGN with the central stationary black hole. The meaningless singularity occurs at the center inside the black hole. (b) The microscopic model of AGN with the central stable SPC. In due course, the neutrinos of huge energies may escape through the vista to outside world. Accepted notations: $\mathrm{EH}=$ event horizon, $\mathrm{AD}=$ accretion disk, $\mathrm{SPC}=$ superdense protomatter core, $\mathrm{PC}=$ protomatter core.

necessary to study the accretion onto central supermassive SPC. The main features of spherical accretion can be briefly summed up in the following three idealized models that illustrate some of the associated physics [72].

5.1. Free Radial Infall. We examine the motion of freely moving test particle by exploring the external geometry of the SPC, with the line element (A.7), at $x=0$. Let us denote the 4vector of velocity of test particle $v^{\mu}=d \widetilde{x}^{\mu} / d \widetilde{s}, \widetilde{x}^{\mu}=(\widetilde{t}, \widetilde{r}, \widetilde{\theta}, \widetilde{\varphi})$, and consider it initially for simplest radial infall $v^{2}=v^{3}=$ 0 . We determine the value of local velocity $v^{\widehat{r}}<0$ of the particle for the moment of crossing the EH sphere, as well as at reaching the surface of central stable SPC. The equation of geodesics is derived from the variational principle $\delta \int d S=0$, which is the extremum of the distance along the wordline for the Lagrangian at hand

$$
2 L=\left(1-x_{0}\right)^{2} \dot{\vec{t}}^{2}-\left(1+x_{0}\right)^{2} \dot{\vec{r}}^{2}-\widetilde{r}^{2} \sin ^{2} \widetilde{\theta} \dot{\tilde{\varphi}}^{2}-\widetilde{r}^{2} \dot{\tilde{\theta}}^{2},
$$

where $\dot{\tilde{t}} \equiv d \tilde{t} / d \lambda$ is the $t$-component of 4-momentum and $\lambda$ is the affine parameter along the worldline. We are using an affine parametrization (by a rescaling $\lambda \rightarrow \lambda\left(\lambda^{\prime}\right)$ ) such that $L=$ const is constant along the curve. A static observer makes measurements with local orthonormal tetrad:

$$
\begin{gathered}
\vec{e}_{\hat{t}}=\left|1-x_{0}\right|^{-1} \vec{e}_{t}, \quad \vec{e}_{\widehat{r}}=\left(1+x_{0}\right)^{-1} \vec{e}_{r}, \\
\vec{e}_{\widehat{\theta}}=\widetilde{r}^{-1} \vec{e}_{\theta}, \quad \vec{e}_{\widehat{\varphi}}=(\widetilde{r} \sin \tilde{\theta})^{-1} \vec{e}_{\theta} .
\end{gathered}
$$

The Euler-Lagrange equations for $\widetilde{\theta}, \tilde{\phi}$, and $\tilde{t}$ can be derived from the variational principle. A local measurement of the particle's energy made by a static observer in the equatorial plane gives the time component of the 4-momentum as measured in the observer's local orthonormal frame. This is the projection of the 4-momentum along the time basis vector. The Euler-Lagrange equations show that if we orient the coordinate system as initially the particle is moving in the equatorial plane (i.e., $\widetilde{\theta}=\pi / 2, \dot{\widetilde{\theta}}=0$ ), then the particle always remains in this plane. There are two constants of the motion corresponding to the ignorable coordinates $\widetilde{t}$ and $\tilde{\phi}$, namely, the $E$-"energy-at-infinity" and the $l$-angular momentum. We conclude that the free radial infall of a particle from the infinity up to the moment of crossing the EH sphere, as well as at reaching the surface of central body, is absolutely the same as in the Schwarzschild geometry of black hole (Figure 2(a)). We clear up a general picture of orbits just outside the event horizon by considering the Euler-Lagrange equation for radial component with "effective potential." The circular orbits are stable if $V$ is concave up, namely, at $\widetilde{r}>4 \widetilde{M}$, where $\widetilde{M}$ is the mass of SPC. The binding energy per unit mass of a particle in the last stable circular orbit at $\widetilde{r}=4 \widetilde{M}$ is $\widetilde{E}_{\text {bind }}=(m-E) / \widetilde{M} \simeq 1-(27 / 32)^{1 / 2}$. Namely, this is the fraction of rest-mass energy released when test particle originally at rest at infinity spirals slowly toward the SPC to the innermost stable circular orbit and then plunges into it. Thereby one of the important parameters is the capture cross section for particles falling in from infinity: $\sigma_{\text {capt }}=\pi b_{\max }^{2}$, where $b_{\max }$ is the maximum impact parameter of a particle that is captured.

5.2. Collisionless Accretion. The distribution function for a collisionless gas is determined by the collisionless Boltzmann equation or Vlasov equation. For the stationary and spherical flow we obtain then

$$
\dot{M}(E>0)=16 \pi(G \widetilde{M})^{2} \rho_{\infty} v_{\infty}^{-1} c^{-2}
$$




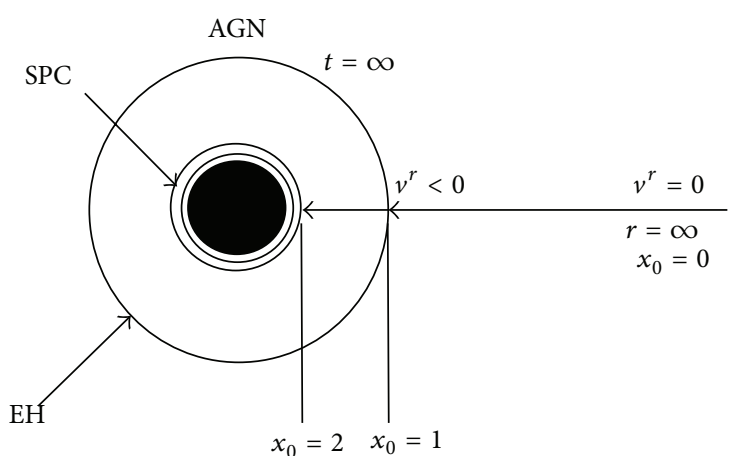

(a)

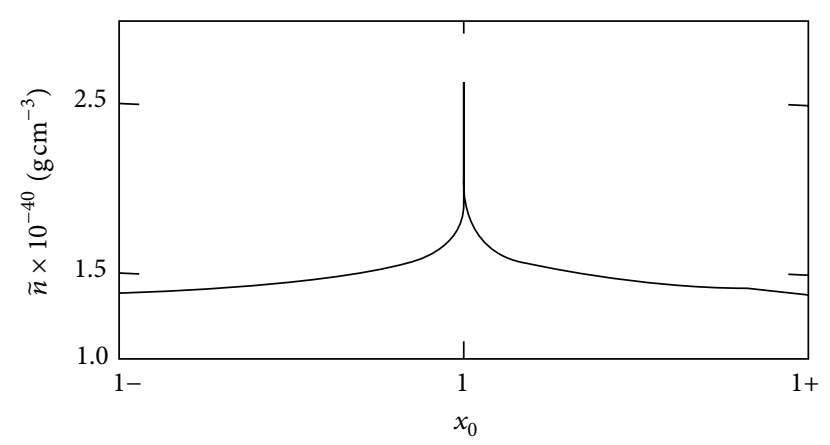

(b)

Figure 2: (a) The free radial infall of a particle from the infinity to EH sphere $\left(x_{0}=1\right)$, which is similar to the Schwarzschild geometry of BH. Crossing the EH sphere, a particle continues infall reaching finally the surface $\left(x_{0}=2\right)$ of the stable SPC. (b) Approaching the EH sphere $\left(x_{0}=1\right)$, the particle concentration increases asymptotically until the threshold value of protomatter. Then, due to the action of cutoff effect, the metric singularity vanishes and the particles well pass EH sphere.

where the particle density $\rho_{\infty}$ is assumed to be uniform at far from the SPC and the particle speed is $v_{\infty} \ll 1$. During the accretion process the particles approaching the $\mathrm{EH}$ become relativistic. Approaching event horizon, the particle concentration increases asymptotically as $\left(\widetilde{n}(\widetilde{r}) / n_{\infty}\right)_{x_{0} \rightarrow 1} \approx$ $-\left(\ln \widetilde{g}_{00}\right) / 2 v_{\infty}$, up to the ID threshold value $\widetilde{n}_{d}(\widetilde{r})^{-1 / 3}=$ $0.4 \mathrm{fm}$ (Figure 2(b)). Due to the action of cutoff effect, the metric singularity then vanishes and the particles well pass $\mathrm{EH}$ sphere $\left(x_{0}=1\right)$ and in the sequel form the protomatter disk around the protomatter core.

5.3. Hydrodynamic Accretion. For real dynamical conditions found in considered superdense medium, it is expected that the mean free path for collisions will be much shorter than the characteristic length scale; that is, the accretion of ambient gas onto a stationary, nonrotating compact SPC will be hydrodynamical in nature. For any equation of state obeying the causality constraint the sound speed implies $a^{2}<1$ and the flow must pass through a critical sonic point $r_{s}$ outside the event horizon. The locally measured particle velocity reads $v^{\widehat{r}}=\left(1-\tilde{g}_{00} / E^{2}\right)$, where $E=E_{\infty} / m=\left(\tilde{g}_{00} /\left(1-u^{2}\right)\right)^{1 / 2}$ and $E_{\infty}$ is the energy at infinity of individual particle of the mass $m$. Thus, the proper flow velocity $v^{\widehat{r}}=u \rightarrow 0$ and is subsonic. At $\widetilde{r}=R_{g} / 2$, the proper velocity equals the speed of light $\left|v^{\widehat{r}}\right|=u=1>a$ and the flow is supersonic. This condition is independent of the magnitude of $u$ and is not sufficient by itself to guarantee that the flow passes through a critical point outside EH. For large $\widetilde{r} \geq r_{s}$, it is expected that the particles be nonrelativistic with $a \leq a_{s} \ll 1$ (i.e., $T \ll m c^{2} / K=10^{13} K$ ), as they were nonrelativistic at infinity $\left(a_{\infty} \ll 1\right)$. Considering the equation of accretion onto superdense protomatter core, which is an analogue of Bondi equations for spherical, steadystate adiabatic accretion onto the SPC, we determine a mass accretion rate

$$
\dot{M}=2 \pi m n_{s} r_{s}^{5 / 2}\left(\ln \widetilde{g}_{00}\right)_{s}^{\prime},
$$

where prime $\left({ }^{\prime}\right)_{s}$ denotes differentiation with respect to $\tilde{r}$ at the point $r_{s}$. The gas compression can be estimated as

$$
\frac{\tilde{n}}{n_{\infty}} \approx \frac{r_{s}^{5 / 2}}{2 r^{2}}\left[\frac{\left(\ln \widetilde{g}_{00}\right)_{s}^{\prime}}{1+\tilde{g}^{r r}(\tilde{r})}\right]^{1 / 2} .
$$

The approximate equality between the sound speed and the mean particle speed implies that the hydrodynamic accretion rate is larger than the collisionless accretion rate by the large factor $\approx 10^{9}$.

\section{The Intermediate Mass, PRT, and Initial Redshift of Seed Black Hole}

The key objectives of the MTBH framework are then an increase of the mass, $M_{\mathrm{BH}}^{\text {Seed }}$, gravitational radius, $R_{g}^{\text {Seed }}$, and of the seed black hole, $\mathrm{BH}^{\text {Seed }}$, at accretion of outside matter. Thereby an infalling matter forms protomatter disk around protomatter core tapering off faster at reaching the thin edge of event horizon. So, a practical measure of growth $\mathrm{BH}^{\text {Seed }} \rightarrow$ $\mathrm{BH}$ may most usefully be the increase of gravitational radius or mass of black hole:

$$
\begin{aligned}
& \Delta R_{g}=R_{g}^{\mathrm{BH}}-R_{g}^{\text {Seed }}=\frac{2 G}{c^{2}} M_{d}=\frac{2 G}{c^{2}} \rho_{d} V_{d}, \\
& \Delta M_{\mathrm{BH}}=M_{\mathrm{BH}}-M_{\mathrm{BH}}^{\text {Seed }}=M_{\mathrm{BH}}^{\text {Seed }} \frac{\Delta R_{g}}{R_{g}^{\text {Seed }}},
\end{aligned}
$$

where $M_{d}, \rho_{d}$, and $V_{d}$, respectively, are the total mass, density, and volume of protomatter disk. At the value $\widehat{R}_{g}^{\mathrm{BH}}$ of gravitational radius, when protomatter disk has finally reached the event horizon of grown-up supermassive black 


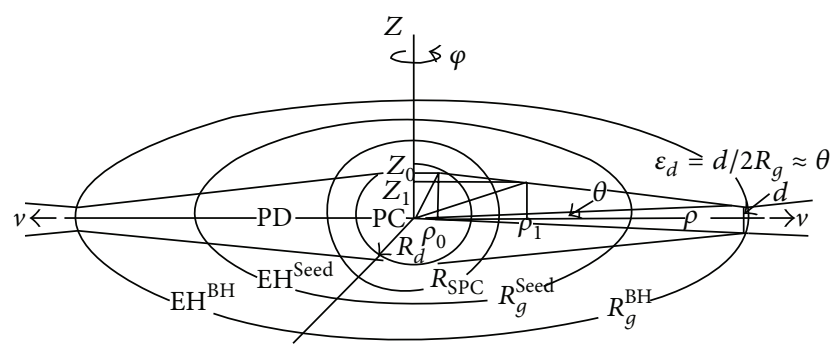

FIGURE 3: A schematic cross section of the growth of supermassive black hole driven by the formation of protomatter disk at accretion, when protomatter disk has finally reached the event horizon of grown-up supermassive black hole.

hole, the volume $\widehat{V}_{d}$ can be calculated in polar coordinates $(\rho, z, \varphi)$ from Figure 3 :

$$
\begin{aligned}
& \widehat{V}_{d}=\int_{\rho_{0}}^{\hat{R}_{g}^{\mathrm{BH}}} d \rho \int_{0}^{2 \pi} \rho d \phi \int_{-z_{1}(\rho)}^{z_{1}(\rho)} d z \\
&-\int_{\rho_{0}}^{R_{d}} d \rho \int_{0}^{2 \pi} \rho d \phi \int_{-z_{0}(\rho)}^{z_{0}(\rho)} d z \\
& \stackrel{\substack{\left(R_{d} \ll \hat{R}_{g}^{\mathrm{BH}} \\
\simeq\right.}}{\simeq} \frac{\sqrt{2} \pi}{3} R_{d}\left(\widehat{R}_{g}^{\mathrm{BH}}\right)^{2},
\end{aligned}
$$

where $z_{1}(\rho) \simeq z_{0}-z_{0}\left(\rho-\rho_{0}\right) /\left(\widehat{R}_{g}^{\mathrm{BH}}-\rho_{0}\right), z_{0}(\rho)=\sqrt{R_{d}^{2}-\rho^{2}}$, and in approximation $R_{d} \ll \widehat{R}_{g}^{\mathrm{BH}}$ we set $z_{0}\left(\rho_{0}\right) \simeq \rho_{0} \simeq R_{d} / \sqrt{2}$.

6.1. The Intermediate Mass of Seed Black Hole. From the first line of (6), by virtue of (7), we obtain

$$
\widehat{R}_{g}^{\mathrm{BH}}=k\left(1 \pm \sqrt{1-\frac{2}{k} R_{g}^{\text {Seed }}}\right),
$$

where $2 / k=8.73[\mathrm{~km}] R_{d} \rho_{d} / M_{\odot}$. The (8) is valid at $(2 /$ k) $R_{g}^{\text {Seed }} \leq 1$; namely,

$$
\frac{R_{\odot}}{R_{d}} \geq 2.09 \frac{[\mathrm{km}]}{R_{\odot}} \frac{\rho_{d}}{\bar{\rho}_{\odot}} \frac{R_{g}^{\text {Seed }}}{R_{\odot}} .
$$

For the values $\rho_{d}=2.6 \times 10^{16}[\mathrm{~g} \mathrm{~cm}]^{-3}$ (see below) and $R_{g}^{\text {Seed }} \simeq$ $2.95[\mathrm{~km}]\left(10^{3}\right.$ to $\left.10^{6}\right)$, inequality (9) is reduced to $R_{\odot} / R_{d} \geq$ $2.34 \times 10^{8}\left(1\right.$ to $\left.10^{3}\right)$ or $[\mathrm{cm}] / R_{d} \geq 0.34\left(10^{-2}\right.$ to 10$)$. This condition is always satisfied, because for considered 377 black holes, with the masses $M_{\mathrm{BH}} / M_{\odot} \simeq 1.1 \times 10^{6}$ to $1.3 \times 10^{10}$, we approximately have $R_{d} / r_{\mathrm{OV}} \simeq 10^{-10}$ to $10^{-7}$ [71]. Note that Woo and Urry [5] collect and compare all the AGN/BH mass and luminosity estimates from the literature. According to (6), the intermediate mass of seed black hole reads

$$
\frac{M_{\mathrm{BH}}^{\mathrm{Seed}}}{M_{\odot}} \simeq \frac{M_{\mathrm{BH}}}{M_{\odot}}\left(1-1.68 \times 10^{-6} \frac{R_{d}}{[\mathrm{~cm}]} \frac{M_{\mathrm{BH}}}{M_{\odot}}\right) .
$$

6.2. $P R T$. The PRT is referred to as a lapse of time $T_{\mathrm{BH}}$ from the birth of black hole till neutrino radiation, the earlier part of the lifetime. That is, $T_{\mathrm{BH}}=M_{d} / \dot{M}$, where $\dot{M}$ is the accretion rate. In approximation at hand $R_{d} \ll R_{g}$, the PRT reads

$$
T_{\mathrm{BH}}=\rho_{d} \frac{V_{d}}{\dot{M}} \simeq 9.33 \cdot 10^{15}\left[\mathrm{~g} \mathrm{~cm}^{-3}\right] \frac{R_{d} R_{g}^{2}}{\dot{M}} .
$$

In case of collisionless accretion, (3) and (11) give

$$
T_{\mathrm{BH}} \simeq 2.6 \cdot 10^{16} \frac{R_{d}}{\mathrm{~cm}} \frac{10^{-24} \mathrm{~g} \mathrm{~cm}^{-3}}{\rho_{\infty}} \frac{v_{\infty}}{10 \mathrm{~km} \mathrm{~s}^{-1}} \mathrm{yr} .
$$

In case of hydrodynamic accretion, (4) and (11) yield

$$
T_{\mathrm{BH}} \simeq 8.8 \cdot 10^{38} \frac{R_{d} R_{g}^{2} \mathrm{~cm}^{-3}}{n_{s} r_{s}^{5 / 2}\left(\ln g_{00}\right)_{s}^{\prime}} .
$$

Note that the spherical accretion onto black hole, in general, is not necessarily an efficient mechanism for converting restmass energy into radiation. Accretion onto black hole may be far from spherical accretion, because the accreted gas possesses angular momentum. In this case, the gas will be thrown into circular orbits about the black hole when centrifugal forces will become significant before the gas plunges through the event horizon. Assuming a typical mass-energy conversion efficiency of about $\epsilon \sim 10 \%$, in approximation $R_{d} \ll R_{g}$, according to (12) and (13), the resulting relationship of typical PRT versus bolometric luminosity becomes

$$
T_{\mathrm{BH}} \simeq 0.32 \frac{R_{d}}{r_{\mathrm{OV}}}\left(\frac{M_{\mathrm{BH}}}{M_{\odot}}\right)^{2} \frac{10^{39} W}{L_{\mathrm{bol}}}[\mathrm{yr}] .
$$

We supplement this by computing neutrino fluxes in the next section.

6.3. Redshift of Seed Black Hole. Interpreting the redshift as a cosmological Doppler effect and that the Hubble law could most easily be understood in terms of expansion of the universe, we are interested in the purely academic question of principle to ask what could be the initial redshift, $z^{\text {Seed }}$, of seed black hole if the mass, the luminosity, and the redshift, $z$, of black hole at present time are known. To follow the history of seed black hole to the present time, let us place ourselves at the origin of coordinates $r=0$ (according to the Cosmological Principle, this is mere convention) and consider a light traveling to us along the $-r$ direction, with angular variables fixed. If the light has left a seed black hole, located at $r_{s}, \theta_{s}$, and $\varphi_{s}$, at time $t_{s}$, and it has to reach us at a time $t_{0}$, then a power series for the redshift as a function of the time of flight is $z^{\text {Seed }}=H_{0}\left(t_{0}-t_{s}\right)+\cdots$, where $t_{0}$ is the present moment and $H_{0}$ is Hubble's constant. Similar expression, $z=H_{0}\left(t_{0}-t_{1}\right)+\cdots$, can be written for the current black hole, located at $r_{1}, \theta_{1}$, and $\varphi_{1}$, at time $t_{1}$, where $t_{1}=$ $t_{s}+T_{\mathrm{BH}}$, as seed black hole is an object at early times. Hence, in the first-order approximation by Hubble's constant, we may obtain the following relation between the redshifts of seed 
and present black holes: $z^{\text {Seed }} \simeq z+H_{0} T_{\mathrm{BH}}$. This relation is in agreement with the scenario of a general recession of distant galaxies away from us in all directions, the furthest naturally being those moving the fastest. This relation, incorporating with (14), for the value $H_{0}=70[\mathrm{~km}] /[\mathrm{s} \mathrm{Mpc}]$ favored today yields

$$
z^{\text {Seed }} \simeq z+2.292 \times 10^{28} \frac{R_{d}}{r_{\mathrm{OV}}}\left(\frac{M_{\mathrm{BH}}}{M_{\odot}}\right)^{2} \frac{W}{L_{\mathrm{bol}}} .
$$

\section{UHE Neutrino Fluxes}

The flux can be written in terms of luminosity as $J_{\nu \varepsilon}=$ $\widetilde{L}_{\nu \mathcal{E}} / 4 \pi D_{L}^{2}(z)(1+z)$, where $z$ is the redshift and $D_{L}(z)$ is the luminosity distance depending on the cosmological model. The $(1+z)^{-1}$ is due to the fact that each neutrino with energy $\widetilde{E}_{\gamma}^{\prime}$ if observed near the place and time of emission $t^{\prime}$ will be red-shifted to energy $\widetilde{E}_{v}=\widetilde{E}_{\gamma}^{\prime} R\left(t_{1}\right) / R\left(t_{0}\right)=\widetilde{E}_{\gamma}^{\prime}(1+z)^{-1}$ of the neutrino observed at time $t$ after its long journey to us, where $R(t)$ is the cosmic scale factor. Computing the UHE neutrino fluxes in the framework of $\mathrm{MTBH}$, we choose the cosmological model favored today, with a flat universe, filled with matter $\Omega_{M}=\rho_{M} / \rho_{c}$ and vacuum energy densities $\Omega_{V}=$ $\rho_{V} / \rho_{c}$, thereby $\Omega_{V}+\Omega_{M}=1$, where the critical energy density $\rho_{c}=3 H_{0}^{2} /\left(8 \pi G_{N}\right)$ is defined through the Hubble parameter $H_{0}[77]$ :

$$
\begin{aligned}
D_{L}(z) & =\frac{(1+z) c}{H_{0} \sqrt{\Omega_{M}}} \int_{1}^{1+z} \frac{d x}{\sqrt{\Omega_{V} / \Omega_{M}+x^{3}}} \\
& =2.4 \times 10^{28} I(z) \mathrm{cm} .
\end{aligned}
$$

Here $I(z)=(1+z) \int_{1}^{1+z} d x / \sqrt{2.3+x^{3}}$, we set the values $H_{0}=$ $70 \mathrm{~km} / \mathrm{s} \mathrm{Mpc}, \Omega_{V}=0.7$ and $\Omega_{M}=0.3$.

7.1. URCA Reactions. The neutrino luminosity of SPC of given mass, $\widetilde{M}$, by modified URCA reactions with no muons reads [75]

$$
\widetilde{L}_{\nu \varepsilon}^{\mathrm{URCA}}=3.8 \times 10^{50} \varepsilon_{d}\left(\frac{M_{\odot}}{\widetilde{M}}\right)^{1.75}\left[\mathrm{erg} \mathrm{s}^{-1}\right],
$$

where $\varepsilon_{d}=d / 2 R_{g}$ and $d$ is the thickness of the protomatter disk at the edge of even horizon. The resulting total UHE neutrino flux of cooling of the SPC can be obtained as

$$
\begin{aligned}
J_{\nu \mathcal{E}}^{\mathrm{URCA}} \simeq & 5.22 \times 10^{-8} \\
& \times \frac{\varepsilon_{d}}{I^{2}(z)(1+z)}\left(\frac{M_{\odot}}{\widetilde{M}}\right)^{1.75}\left[\mathrm{erg} \mathrm{cm}^{-2} \mathrm{~s}^{-1} \mathrm{sr}^{-1}\right]
\end{aligned}
$$

where the neutrino is radiated in a cone with the beaming angle $\theta \sim \varepsilon_{d} \ll 1, I(z)=(1+z) \int_{1}^{1+z} d x / \sqrt{2.3+x^{3}}$. As it is seen, the nucleon modified URCA reactions can contribute efficiently only to extragalactic objects with enough small redshift $z \ll 1$.
7.2. Pionic Reactions. The pionic reactions, occurring in the superdense protomatter medium of SPC, allow both the distorted energy and momentum to be conserved. This is the analogue of the simple URCA processes:

$$
\pi^{-}+n \longrightarrow n+e^{-}+\bar{\nu}_{e}, \quad \pi^{-}+n \longrightarrow n+\mu^{-}+\bar{\nu}_{\mu}
$$

and the two inverse processes. As in the modified URCA reactions, the total rate for all four processes is essentially four times the rate of each reaction alone. The muons are already present when pions appear. The neutrino luminosity of the SPC of given mass, $\widetilde{M}$, by pionic reactions reads [75]

$$
\widetilde{L}_{\nu \varepsilon}^{\pi}=5.78 \times 10^{58} \varepsilon_{d}\left(\frac{M_{\odot}}{\widetilde{M}}\right)^{1.75}\left[\mathrm{erg} \mathrm{s}^{-1}\right] .
$$

Then, the UHE neutrino total flux is

$$
J_{\nu \varepsilon}^{\pi} \simeq 7.91 \frac{\varepsilon_{d}}{I^{2}(z)(1+z)}\left(\frac{M_{\odot}}{\widetilde{M}}\right)^{1.75}\left[\mathrm{erg} \mathrm{cm}^{-2} \mathrm{~s}^{-1} \mathrm{sr}^{-1}\right] .
$$

The resulting total energy-loss rate will then be dramatically larger due to the pionic reactions (19) rather than the modified URCA processes.

7.3. Quark Reactions. In the superdense protomatter medium the distorted quark Fermi energies are far below the charmed $c^{-}, \mathrm{t}-$, and b-quark production thresholds. Therefore, only up-, down-, and strange quarks are present. The $\beta$ equilibrium is maintained by reactions like

$$
\begin{array}{ll}
d \longrightarrow u+e^{-}+\bar{v}_{e}, & u+e^{-} \longrightarrow d+v_{e}, \\
s \longrightarrow u+e^{-}+\bar{v}_{e}, & u+e^{-} \longrightarrow s+v_{e},
\end{array}
$$

which are $\beta$ decay and its inverse. These reactions constitute simple URCA processes, in which there is a net loss of a $\nu_{l} \bar{\nu}_{l}$ pair at nonzero temperatures. In this application a sufficient accuracy is obtained by assuming $\beta$-equilibrium and that the neutrinos are not retained in the medium of $\Lambda$-like protomatter. The quark reactions (22) and (23) proceed at equal rates in $\beta$ equilibrium, where the participating quarks must reside close to their Fermi surface. Hence, the total energy of flux due to simple URCA processes is rather twice than that of (22) or (23) alone. For example, the spectral fluxes of the UHE antineutrinos and neutrinos for different redshifts from quark reactions are plotted, respectively, in Figures 4 and 5 [75]. The total flux of UHE neutrino can be written as

$$
J_{\nu \varepsilon}^{q} \simeq 70.68 \frac{\varepsilon_{d}}{I^{2}(z)(1+z)}\left(\frac{M_{\odot}}{\widetilde{M}}\right)^{1.75}\left[\mathrm{erg} \mathrm{cm}^{-2} \mathrm{~s}^{-1} \mathrm{sr}^{-1}\right] .
$$

\section{Simulation}

For simulation we use the data of AGN/BH mass and luminosity estimates for 377 black holes presented by [5]. These masses are mostly based on the virial assumption for the broad emission lines, with the broad-line region size 

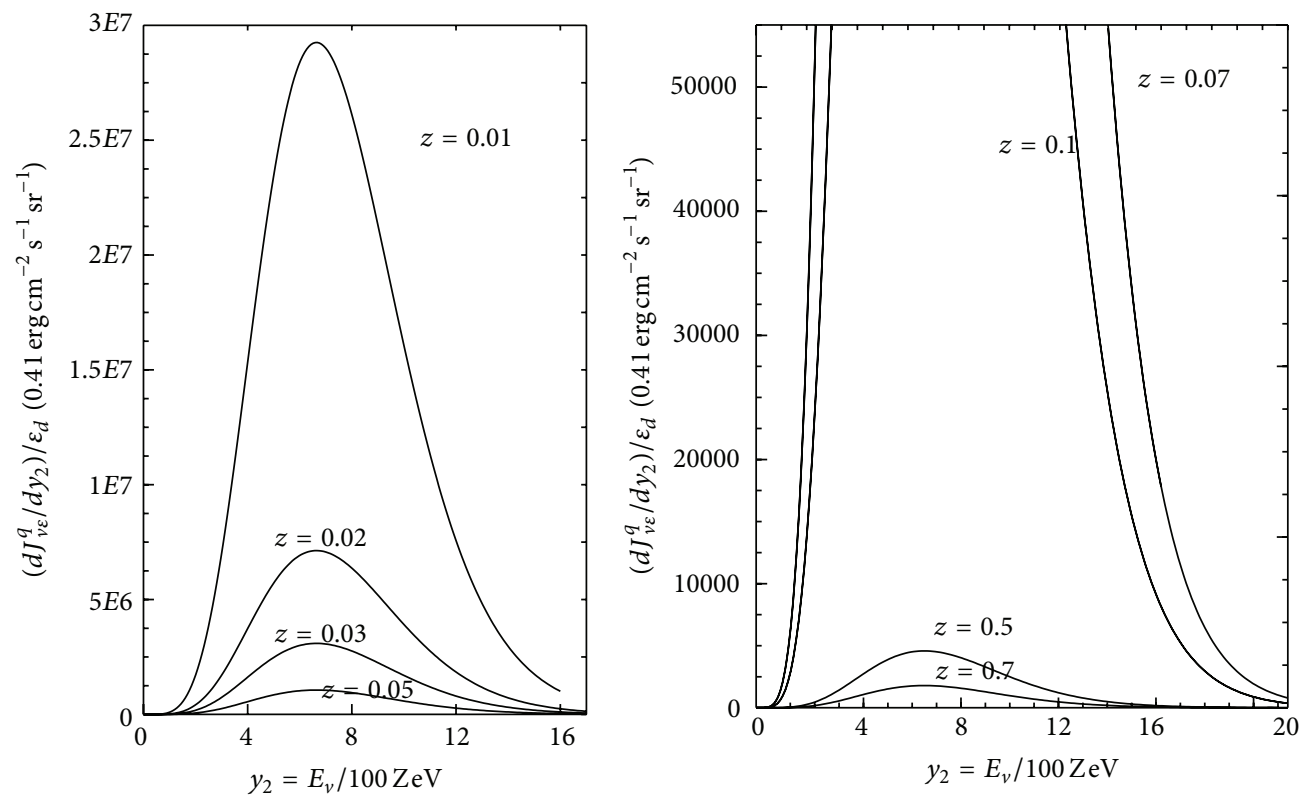

FIgURE 4: The spectral fluxes of UHE antineutrinos for different redshifts from quark reactions.
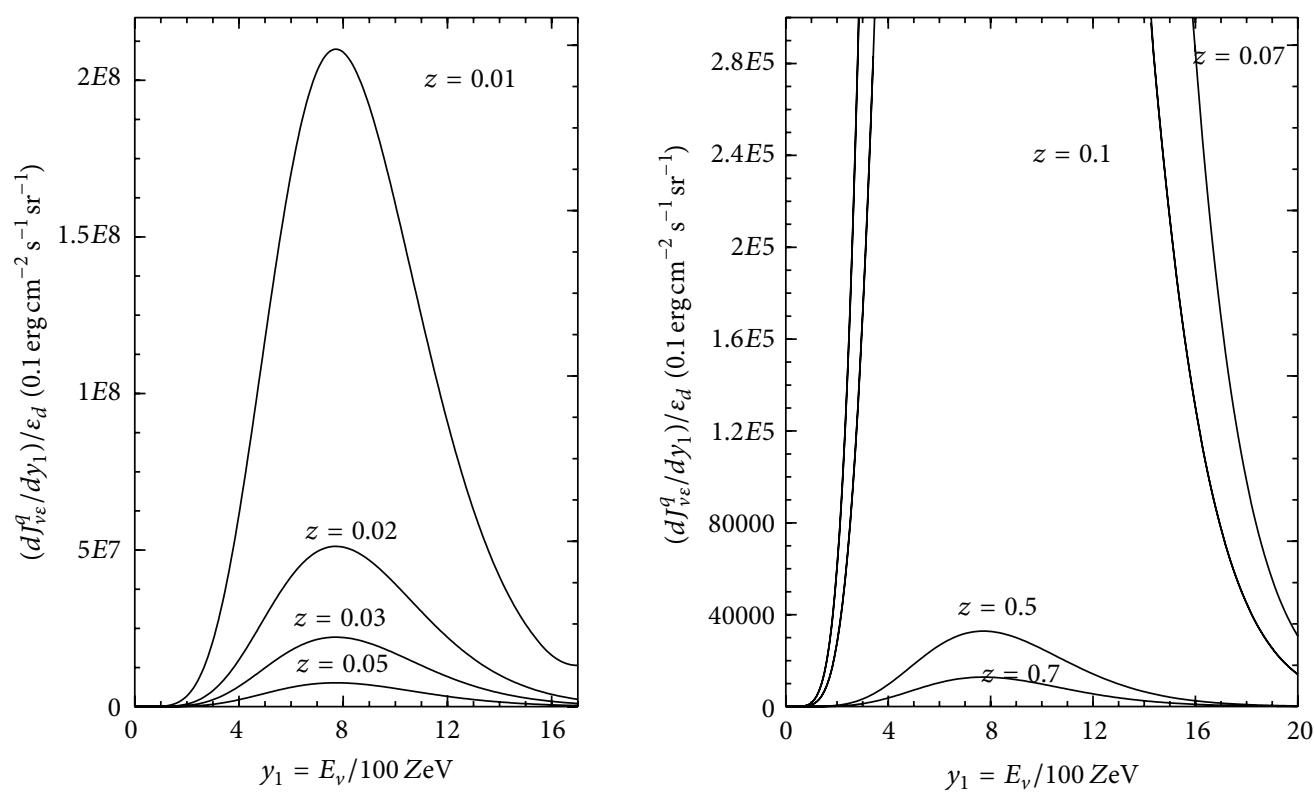

FIGURE 5: The spectral fluxes of UHE neutrinos for different redshifts from quark reactions.

determined from either reverberation mapping or optical luminosity. Additional black hole mass estimates based on properties of the host galaxy bulges, either using the observed stellar velocity dispersion or using the fundamental plane relation. Since the aim is to have more than a thousand of realizations, each individual run is simplified, with a use of previous algorithm of the SPC-configurations [71] as a working model, given in Appendix G. Computing the corresponding PRTs, seed black hole intermediate masses, and total neutrino fluxes, a main idea comes to solving an inverse problem. Namely, by the numerous reiterating integrations of the state equations of SPC-configurations we determine those required central values of particle concentration $\widetilde{n}(0)$ and IDfield $x(0)$, for which the integrated total mass of configuration has to be equal to the black hole mass $M_{\mathrm{BH}}$ given from observations. Along with all integral characteristics, the radius $R_{d}$ is also computed, which is further used in (10), (14), (15), (18), (21), and (24) for calculating $M_{\mathrm{BH}}^{\text {Seed }}, T_{\mathrm{BH}}, z^{\text {Seed }}$, and $J_{v \mathcal{E}}^{i}$, respectively. The results are summed up in Tables 1, 2, 3, 4 and 5. Figure 6 gives the intermediate seed masses $M_{\mathrm{BH}}^{\text {Seed }} / M_{\odot}$ versus the present masses $M_{\mathrm{BH}} / M_{\odot}$ of 337 black holes, on logarithmic scales. For the present masses $M_{\mathrm{BH}} / M_{\odot} \simeq 1.1 \times$ $10^{6}$ to $1.3 \times 10^{10}$, the computed intermediate seed masses 


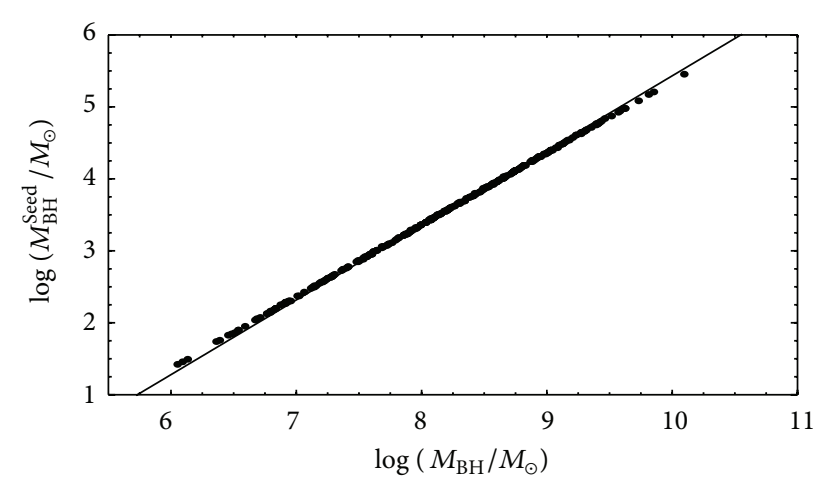

FIGURE 6: The $M_{\mathrm{BH}}^{\text {Seed }} / M_{\odot}-M_{\mathrm{BH}} / M_{\odot}$ relation on logarithmic scales of 337 black holes from [5]. The solid line is the best fit to data of samples.

are ranging from $M_{\mathrm{BH}}^{\text {Seed }} / M_{\odot} \simeq 26.4$ to $2.9 \times 10^{5}$. The computed neutrino fluxes are ranging from (1) (quark reactions) $-J_{\nu \varepsilon}^{q} / \varepsilon_{d}\left[\mathrm{erg} \mathrm{cm}^{-2} \mathrm{~s}^{-1} \mathrm{sr}^{-1}\right] \simeq 8.29 \times 10^{-16}$ to $3.18 \times$ $10^{-4}$, with the average $\bar{J}_{v \varepsilon}^{q} \simeq 5.53 \times 10^{-10} \varepsilon_{d}\left[\mathrm{erg} \mathrm{cm} \mathrm{cm}^{-2} \mathrm{sr}^{-1}\right]$; (2) (pionic reactions) $-J_{\nu \varepsilon}^{\pi} \simeq 0.112 J_{\nu \varepsilon}^{q}$, with the average $J_{\nu \mathcal{E}}^{\pi} \simeq 3.66 \times 10^{-11} \varepsilon_{d}\left[\mathrm{erg} \mathrm{cm}^{-2} \mathrm{~s}^{-1} \mathrm{sr}^{-1}\right]$; and (3) (modified URCA processes) $-J_{\nu \mathcal{E}}^{\mathrm{URCA}} \simeq 7.39 \times 10^{-11} J_{\nu \mathcal{E}}^{q}$, with the average $\bar{J}_{\nu \varepsilon}^{\mathrm{URCA}} \simeq 2.41 \times 10^{-20} \varepsilon_{d}\left[\mathrm{erg} \mathrm{cm}^{-2} \mathrm{~s}^{-1} \mathrm{sr}^{-1}\right]$. In accordance, the AGNs are favored as promising pure neutrino sources because the computed neutrino fluxes are highly beamed along the plane of accretion disk and peaked at high energies and collimated in smaller opening angle $\theta \sim \varepsilon_{d}=d / 2 r_{g} \ll 1$. To render our discussion here a bit more transparent and to obtain some feeling for the parameter $\varepsilon_{d}$ we may estimate $\varepsilon_{d} \simeq 1.69 \times 10^{-10}$, just for example, only, for the suppermassive black hole of typical mass $\sim 10^{9} M_{\odot}\left(2 R_{g}=5.9 \times 10^{14} \mathrm{~cm}\right)$, and so $d \sim 1 \mathrm{~km}$. But the key problem of fixing the parameter $\varepsilon_{d}$ more accurately from experiment would be an important topic for another investigation elsewhere.

\section{Conclusions}

The growth of accreting supermassive black hole seeds and their neutrino radiation are found to be common phenomena in the AGNs. In this report, we further expose the assertions made in the framework of microscopic theory of black hole via reviewing the mass assembly history of 377 plausible accreting supermassive black hole seeds. After the numerous reiterating integrations of the state equations of SPC-configurations, we compute their intermediate seed masses, $M_{\mathrm{BH}}^{\text {Seed }}$, PRTs, initial redshifts, $z^{\text {Seed }}$, and neutrino fluxes. All the results are presented in Tables 1-5. Figure 6 gives the intermediate seed masses $M_{\mathrm{BH}}^{\text {Seed }} / M_{\odot}$ versus the present masses $M_{\mathrm{BH}} / M_{\odot}$ of 337 black holes, on logarithmic scales. In accordance, the AGNs are favored as promising pure UHE neutrino sources. Such neutrinos may reveal clues on the puzzle of origin of UHE cosmic rays. We regard the considered black holes only as the potential neutrino sources. The obtained results, however, may suffer and that would be underestimated if not all 377 live black holes in the
$M_{\mathrm{BH}} / M_{\odot} \simeq 1.1 \times 10^{6}$ to $1.3 \times 10^{10}$ mass range at present reside in final stage of their growth, when the protomatter disk driven by accretion has reached the event horizon.

\section{Appendices}

\section{A. Outline of the Key Points of Proposed Gravitation Theory at Huge Energies}

The proposed gravitation theory explores the most important processes of rearrangement of vacuum state and a spontaneous breaking of gravitation gauge symmetry at huge energies. From its historical development, the efforts in gauge treatment of gravity mainly focus on the quantum gravity and microphysics, with the recent interest, for example, in the theory of the quantum superstring or, in the very early universe, in the inflationary model. The papers on the gauge treatment of gravity provide a unified picture of gravity modified models based on several Lie groups. However, currently no single theory has been uniquely accepted as the convincing gauge theory of gravitation which could lead to a consistent quantum theory of gravity. They have evoked the possibility that the treatment of spacetime might involve nonRiemannian features on the scale of the Planck length. This necessitates the study of dynamical theories involving postRiemannian geometries. It is well known that the notions of space and connections should be separated; see, for example, [78-81]. The curvature and torsion are in fact properties of a connection, and many different connections are allowed to exist in the same spacetime. Therefore, when considering several connections with different curvature and torsion, one takes spacetime simply as a manifold and connections as additional structures. From this view point in a recent paper [82] we tackle the problem of spacetime deformation. This theory generalizes and, in particular cases, fully recovers the results of the conventional theory. Conceptually and techniquewise this theory is versatile and powerful and manifests its practical and technical virtue in the fact that through a nontrivial choice of explicit form of the world-deformation tensor, which we have at our disposal, in general, we have a way to deform the spacetime which displayed different connections, which may reveal different post-Riemannian spacetime structures as corollary. All the fundamental gravitational structures in fact-the metric as much as the coframes and connections-acquire a spacetime deformation induced theoretical interpretation. There is another line of reasoning which supports the side of this method. We address the theory of teleparallel gravity and construct a consistent Einstein-Cartan (EC) theory with the dynamical torsion. We show that the equations of the standard EC theory, in which the equation defining torsion is the algebraic type and, in fact, no propagation of torsion is allowed, can be equivalently replaced by the set of modified Einstein-Cartan equations in which the torsion, in general, is dynamical. Moreover, the special physical constraint imposed upon the spacetime deformations yields the short-range propagating spin-spin interaction. For the self-contained arguments in Appendix A.1 and Appendices B and C we complete the 
TABLE 1: Seed black hole intermediate masses, preradiation times, redshifts, and neutrino fluxes from spatially resolved kinematics. Columns: (1) name, (2) redshift, (3) AGN type: SY2: Seyfert 2, (4) log of the bolometric luminosity ( $\mathrm{ergs}^{-1}$ ), (5) log of the radius of protomatter core in special unit $r_{\mathrm{OV}}=13.68 \mathrm{~km},(6) \log$ of the black hole mass in solar masses, (7) $\log$ of the seed black hole intermediate mass in solar masses, (8) log of the neutrino preradiation time (yrs), (9) redshift of seed black hole, (10) $J^{i=q}$, (11) $J^{i=\text { URCA }}$, and (12) $J^{i=\pi}$, where $J^{i} \equiv \log \left(J_{v \varepsilon}^{i} / \varepsilon_{d} \operatorname{erg~cm}^{-2} \mathrm{~s}^{-1} \mathrm{sr}^{-1}\right)$.

\begin{tabular}{|c|c|c|c|c|c|c|c|c|c|c|c|}
\hline Name & $z$ & Type & $\log L_{\text {bol }}$ & $\log \left(\frac{R_{d}}{r_{\mathrm{ov}}}\right)$ & $\log \left(\frac{M_{\mathrm{BH}}}{M_{\odot}}\right)$ & $\log \left(\frac{M_{\mathrm{BH}}^{\text {Seed }}}{M_{\odot}}\right)$ & $\log T_{\mathrm{BH}}$ & $z^{\text {Seed }}$ & $J^{q}$ & $J^{\mathrm{URCA}}$ & $J^{\pi}$ \\
\hline NGC 1068 & 0.004 & SY2 & 44.98 & -7.59201 & 7.23 & 2.5922 & 8.02 & 0.006 & -5.49 & -15.62 & -6.44 \\
\hline NGC 4258 & 0.001 & SY2 & 43.45 & -7.98201 & 7.62 & 2.9822 & 10.33 & 0.148 & -4.97 & -15.10 & -5.92 \\
\hline
\end{tabular}

TABLE 2: Seed black hole intermediate masses, preradiation times, redshifts, and neutrino fluxes from reverberation mapping. Columns: (1) name, (2) redshift, (3) AGN type: SY2: Seyfert 2, (4) log of the bolometric luminosity ( $\mathrm{ergs}^{-1}$ ), (5) log of the radius of protomatter core in special unit $r_{\mathrm{OV}}=13.68 \mathrm{~km},(6) \log$ of the black hole mass in solar masses, (7) log of the seed black hole intermediate mass in solar masses, (8) log of the neutrino preradiation time (yrs), (9) redshift of seed black hole, (10) $J^{i=q}$, (11) $J^{i=\mathrm{URCA}}$, and (12) $J^{i=\pi}$, where $J^{i} \equiv \log \left(J_{v \varepsilon}^{i} / \varepsilon_{d} \operatorname{erg~cm}^{-2} \mathrm{~s}^{-1} \mathrm{sr}^{-1}\right)$.

\begin{tabular}{|c|c|c|c|c|c|c|c|c|c|c|c|}
\hline Name & $z$ & Type & $\log L_{\text {bol }}$ & $\log \left(\frac{R_{d}}{r_{\mathrm{ov}}}\right)$ & $\log \left(\frac{M_{\mathrm{BH}}}{M_{\odot}}\right)$ & $\log \left(\frac{M_{\mathrm{BH}}^{\text {Seed }}}{M_{\odot}}\right)$ & $\log T_{\mathrm{BH}}$ & $z^{\text {Seed }}$ & $J^{q}$ & $J^{\mathrm{URCA}}$ & $J^{\pi}$ \\
\hline 3C 120 & 0.033 & SY1 & 45.34 & -7.78201 & 7.42 & 2.7822 & 8.04 & 0.034 & -7.69 & -17.82 & -8.64 \\
\hline 3C 390.3 & 0.056 & SY1 & 44.88 & -8.91201 & 8.55 & 3.9122 & 10.76 & 0.103 & -10.15 & -20.28 & -11.10 \\
\hline Akn 120 & 0.032 & SY1 & 44.91 & -8.63201 & 8.27 & 3.6322 & 10.17 & 0.055 & -9.15 & -19.28 & -10.10 \\
\hline F9 & 0.047 & SY1 & 45.23 & -8.27201 & 7.91 & 3.2722 & 9.13 & 0.052 & -8.87 & -19.00 & -9.82 \\
\hline IC 4329A & 0.016 & SY1 & 44.78 & -7.13201 & 6.77 & 2.1322 & 7.30 & 0.017 & -5.91 & -16.04 & -6.86 \\
\hline Mrk 79 & 0.022 & SY1 & 44.57 & -8.22201 & 7.86 & 3.2222 & 9.69 & 0.041 & -8.10 & -18.23 & -9.05 \\
\hline Mrk 110 & 0.035 & SY1 & 44.71 & -7.18201 & 6.82 & 2.1822 & 7.47 & 0.036 & -6.69 & -16.82 & -7.64 \\
\hline Mrk 335 & 0.026 & SY1 & 44.69 & -7.05201 & 6.69 & 2.0522 & 7.23 & 0.027 & -6.20 & -16.33 & -7.15 \\
\hline Mrk 509 & 0.034 & SY1 & 45.03 & -8.22201 & 7.86 & 3.2222 & 9.23 & 0.041 & -8.49 & -18.62 & -9.44 \\
\hline Mrk 590 & 0.026 & SY1 & 44.63 & -7.56201 & 7.20 & 2.5622 & 8.31 & 0.030 & -7.09 & -17.22 & -8.04 \\
\hline Mrk 817 & 0.032 & SY1 & 44.99 & -7.96201 & 7.60 & 2.9622 & 8.75 & 0.036 & -7.98 & -18.11 & -8.93 \\
\hline NGC 3227 & 0.004 & SY1 & 43.86 & -8.00201 & 7.64 & 3.0022 & 9.96 & 0.064 & -6.21 & -16.34 & -7.16 \\
\hline NGC 3516 & 0.009 & SY1 & 44.29 & -7.72201 & 7.36 & 2.7222 & 8.97 & 0.021 & -6.43 & -16.56 & -7.38 \\
\hline NGC 3783 & 0.010 & SY1 & 44.41 & -7.30201 & 6.94 & 2.3022 & 8.01 & 0.013 & -5.79 & -15.92 & -6.74 \\
\hline NGC 4051 & 0.002 & SY1 & 43.56 & -6.49201 & 6.13 & 1.4922 & 7.24 & 0.006 & -2.96 & -13.10 & -3.91 \\
\hline NGC 4151 & 0.003 & SY1 & 43.73 & -7.49201 & 7.13 & 2.4922 & 9.07 & 0.028 & -5.07 & -15.20 & -6.02 \\
\hline NGC 4593 & 0.009 & SY1 & 44.09 & -7.27201 & 6.91 & 2.2722 & 8.27 & 0.016 & -5.64 & -15.77 & -6.59 \\
\hline NGC 5548 & 0.017 & SY1 & 44.83 & -8.39201 & 8.03 & 3.3922 & 9.77 & 0.033 & -8.16 & -18.30 & -9.11 \\
\hline NGC 7469 & 0.016 & SY1 & 45.28 & -7.20201 & 6.84 & 2.2022 & 6.94 & 0.016 & -6.03 & -16.16 & -6.98 \\
\hline PG $0026+129$ & 0.142 & RQQ & 45.39 & -7.94201 & 7.58 & 2.9422 & 8.31 & 0.144 & -9.35 & -19.48 & -10.30 \\
\hline PG 0052 + 251 & 0.155 & RQQ & 45.93 & -8.77201 & 8.41 & 3.7722 & 9.43 & 0.158 & -10.89 & -21.02 & -11.84 \\
\hline PG $0804+761$ & 0.100 & RQQ & 45.93 & -8.60201 & 8.24 & 3.6022 & 9.09 & 0.102 & -10.16 & -20.29 & -11.11 \\
\hline PG $0844+349$ & 0.064 & RQQ & 45.36 & -7.74201 & 7.38 & 2.7422 & 7.94 & 0.065 & -8.23 & -18.36 & -9.18 \\
\hline PG 0953 + 414 & 0.239 & RQQ & 46.16 & -8.60201 & 8.24 & 3.6022 & 8.86 & 0.240 & -11.04 & -21.17 & -11.99 \\
\hline PG 1211 + 143 & 0.085 & RQQ & 45.81 & -7.85201 & 7.49 & 2.8522 & 7.71 & 0.085 & -8.69 & -18.82 & -9.64 \\
\hline PG $1229+204$ & 0.064 & RQQ & 45.01 & -8.92201 & 8.56 & 3.9222 & 10.65 & 0.099 & -10.29 & -20.42 & -11.24 \\
\hline PG 1307 + 085 & 0.155 & RQQ & 45.83 & -8.26201 & 7.90 & 3.2622 & 8.51 & 0.156 & -9.99 & -20.12 & -10.94 \\
\hline PG $1351+640$ & 0.087 & RQQ & 45.50 & -8.84201 & 8.48 & 3.8422 & 10.00 & 0.097 & -10.44 & -20.57 & -11.39 \\
\hline PG $1411+442$ & 0.089 & RQQ & 45.58 & -7.93201 & 7.57 & 2.9322 & 8.10 & 0.090 & -8.87 & -19.00 & -9.82 \\
\hline PG $1426+015$ & 0.086 & RQQ & 45.19 & -8.28201 & 7.92 & 3.2822 & 9.19 & 0.091 & -9.45 & -19.58 & -10.40 \\
\hline PG $1613+658$ & 0.129 & RQQ & 45.66 & -8.98201 & 8.62 & 3.9822 & 10.12 & 0.138 & -11.07 & -21.20 & -12.02 \\
\hline PG 1617 + 175 & 0.114 & RQQ & 45.52 & -8.24201 & 7.88 & 3.2422 & 8.78 & 0.116 & -9.65 & -19.78 & -10.60 \\
\hline PG 1700 + 518 & 0.292 & RQQ & 46.56 & -8.67201 & 8.31 & 3.6722 & 8.60 & 0.293 & -11.38 & -21.51 & -12.33 \\
\hline PG $2130+099$ & 0.061 & RQQ & 45.47 & -8.10201 & 7.74 & 3.1022 & 8.55 & 0.063 & -8.81 & -18.94 & -9.76 \\
\hline PG $1226+023$ & 0.158 & RLQ & 47.35 & -7.58201 & 7.22 & 2.5822 & 5.63 & 0.158 & -8.82 & -18.95 & -9.77 \\
\hline PG $1704+608$ & 0.371 & RLQ & 46.33 & -8.59201 & 8.23 & 3.5922 & 8.67 & 0.372 & -11.50 & -21.64 & -12.45 \\
\hline
\end{tabular}


TABLE 3: Seed black hole intermediate masses, preradiation times, redshifts, and neutrino fluxes from optimal luminosity. Columns: (1) name, (2) redshift, (3) AGN type: SY2: Seyfert 2, (4) log of the bolometric luminosity ( $\mathrm{ergs}^{-1}$ ), (5) log of the radius of protomatter core in special unit $r_{\mathrm{OV}}=13.68 \mathrm{~km},(6) \log$ of the black hole mass in solar masses, (7) log of the seed black hole intermediate mass in solar masses, (8) log of the neutrino preradiation time (yrs), (9) redshift of seed black hole, (10) $J^{i=q}$, (11) $J^{i=\mathrm{URCA}}$, and (12) $J^{i=\pi}$, where $J^{i} \equiv \log \left(J_{v \varepsilon}^{i} / \varepsilon_{d} \mathrm{erg} \mathrm{cm}^{-2} \mathrm{~s}^{-1} \mathrm{sr}^{-1}\right)$.

\begin{tabular}{|c|c|c|c|c|c|c|c|c|c|c|c|}
\hline Name & $z$ & Type & $\log L_{\text {bol }}$ & $\log \left(\frac{R_{d}}{r_{\mathrm{ov}}}\right)$ & $\log \left(\frac{M_{\mathrm{BH}}}{M_{\odot}}\right)$ & $\log \left(\frac{M_{\mathrm{BH}}^{\text {Seed }}}{M_{\odot}}\right)$ & $\log T_{\mathrm{BH}}$ & $z^{\text {Seed }}$ & $J^{q}$ & $J^{\mathrm{URCA}}$ & $J^{\pi}$ \\
\hline Mrk 841 & 0.036 & SY1 & 45.84 & -8.46201 & 8.10 & 3.4622 & 8.90 & 0.038 & -8.96 & -19.09 & -9.91 \\
\hline NGC 4253 & 0.013 & SY1 & 44.40 & -6.90201 & 6.54 & 1.9022 & 7.22 & 0.014 & -5.32 & -15.45 & -6.27 \\
\hline NGC 6814 & 0.005 & SY1 & 43.92 & -7.64201 & 7.28 & 2.6422 & 9.18 & 0.028 & -5.78 & -15.91 & -6.73 \\
\hline $0054+144$ & 0.171 & RQQ & 45.47 & -9.26201 & 8.90 & 4.2622 & 10.87 & 0.198 & -11.84 & -21.97 & -12.79 \\
\hline $0157+001$ & 0.164 & RQQ & 45.62 & -8.06201 & 7.70 & 3.0622 & 8.32 & 0.165 & -9.70 & -19.83 & -10.65 \\
\hline $0204+292$ & 0.109 & RQQ & 45.05 & -7.03201 & 6.67 & 2.0322 & 6.83 & 0.109 & -7.49 & -17.62 & -8.44 \\
\hline $0205+024$ & 0.155 & RQQ & 45.45 & -8.22201 & 7.86 & 3.2222 & 8.81 & 0.158 & -9.92 & -20.05 & -10.87 \\
\hline $0244+194$ & 0.176 & RQQ & 45.51 & -8.39201 & 8.03 & 3.3922 & 9.09 & 0.179 & -10.35 & -20.48 & -11.30 \\
\hline $0923+201$ & 0.190 & RQQ & 46.22 & -9.30201 & 8.94 & 4.3022 & 10.20 & 0.195 & -12.02 & -22.15 & -12.97 \\
\hline $1012+008$ & 0.185 & RQQ & 45.51 & -8.15201 & 7.79 & 3.1522 & 8.61 & 0.187 & -9.98 & -20.11 & -10.93 \\
\hline $1029-140$ & 0.086 & RQQ & 46.03 & -9.44201 & 9.08 & 4.4422 & 10.67 & 0.097 & -11.48 & -21.61 & -12.43 \\
\hline $1116+215$ & 0.177 & RQQ & 46.02 & -8.57201 & 8.21 & 3.5722 & 8.94 & 0.179 & -10.67 & -20.80 & -11.62 \\
\hline $1202+281$ & 0.165 & RQQ & 45.39 & -8.65201 & 8.29 & 3.6522 & 9.73 & 0.173 & -10.74 & -20.87 & -11.69 \\
\hline $1309+355$ & 0.184 & RQQ & 45.39 & -8.36201 & 8.00 & 3.3622 & 8.91 & 0.186 & -10.34 & -20.47 & -11.29 \\
\hline $1402+261$ & 0.164 & RQQ & 45.13 & -7.65201 & 7.29 & 2.6522 & 7.99 & 0.165 & -8.98 & -19.11 & -9.93 \\
\hline $1444+407$ & 0.267 & RQQ & 45.93 & -8.42201 & 8.06 & 3.4222 & 8.73 & 0.268 & -10.84 & -20.97 & -11.79 \\
\hline $1635+119$ & 0.146 & RQQ & 45.13 & -8.46201 & 8.10 & 3.4622 & 9.61 & 0.155 & -10.28 & -20.41 & -11.23 \\
\hline $0022-297$ & 0.406 & RLQ & 44.98 & -8.27201 & 7.91 & 3.2722 & 9.38 & 0.414 & -11.05 & -21.18 & -12.00 \\
\hline $0024+348$ & 0.333 & RLQ & 45.31 & -6.73201 & 6.37 & 1.7322 & 5.97 & 0.333 & -8.13 & -18.26 & -9.08 \\
\hline $0056-001$ & 0.717 & RLQ & 46.54 & -9.07201 & 8.71 & 4.0722 & 9.42 & 0.718 & -13.13 & -23.26 & -14.08 \\
\hline $0110+495$ & 0.395 & RLQ & 45.78 & -8.70201 & 8.34 & 3.7022 & 9.44 & 0.399 & -11.77 & -21.90 & -12.72 \\
\hline $0114+074$ & 0.343 & RLQ & 44.02 & -7.16201 & 6.80 & 2.1622 & 8.12 & 0.349 & -8.91 & -19.04 & -9.86 \\
\hline $0119+041$ & 0.637 & RLQ & 45.57 & -8.74201 & 8.38 & 3.7422 & 9.73 & 0.643 & -12.41 & -22.54 & -13.36 \\
\hline $0133+207$ & 0.425 & RLQ & 45.83 & -9.88201 & 9.52 & 4.8822 & 11.75 & 0.474 & -13.92 & -24.05 & -14.87 \\
\hline $0133+476$ & 0.859 & RLQ & 46.69 & -9.09201 & 8.73 & 4.0922 & 9.31 & 0.860 & -13.40 & -23.53 & -14.35 \\
\hline $0134+329$ & 0.367 & RLQ & 46.44 & -9.10201 & 8.74 & 4.1022 & 9.58 & 0.369 & -12.38 & -22.52 & -13.33 \\
\hline $0135-247$ & 0.831 & RLQ & 46.64 & -9.49201 & 9.13 & 4.4922 & 10.16 & 0.834 & -14.06 & -24.19 & -15.01 \\
\hline $0137+012$ & 0.258 & RLQ & 45.22 & -8.93201 & 8.57 & 3.9322 & 10.46 & 0.280 & -11.70 & -21.83 & -12.65 \\
\hline $0153-410$ & 0.226 & RLQ & 44.74 & -7.92201 & 7.56 & 2.9222 & 8.92 & 0.233 & -9.79 & -19.92 & -10.74 \\
\hline $0159-117$ & 0.669 & RLQ & 46.84 & -9.63201 & 9.27 & 4.6322 & 10.24 & 0.672 & -14.03 & -24.16 & -14.98 \\
\hline $0210+860$ & 0.186 & RLQ & 44.92 & -6.90201 & 6.54 & 1.9022 & 6.70 & 0.186 & -7.80 & -17.93 & -8.75 \\
\hline $0221+067$ & 0.510 & RLQ & 44.94 & -7.65201 & 7.29 & 2.6522 & 8.18 & 0.512 & -10.23 & -20.36 & -11.18 \\
\hline $0237-233$ & 2.224 & RLQ & 47.72 & -8.88201 & 8.52 & 3.8822 & 7.86 & 2.224 & -14.39 & -24.52 & -15.34 \\
\hline $0327-241$ & 0.888 & RLQ & 46.01 & -8.96201 & 8.60 & 3.9622 & 9.73 & 0.892 & -13.22 & -23.35 & -14.17 \\
\hline $0336-019$ & 0.852 & RLQ & 46.32 & -9.34201 & 8.98 & 4.3422 & 10.18 & 0.857 & -13.83 & -23.96 & -14.78 \\
\hline $0403-132$ & 0.571 & RLQ & 46.47 & -9.43201 & 9.07 & 4.4322 & 10.21 & 0.575 & -13.48 & -23.61 & -14.43 \\
\hline $0405-123$ & 0.574 & RLQ & 47.40 & -9.83201 & 9.47 & 4.8322 & 10.08 & 0.575 & -14.19 & -24.32 & -15.14 \\
\hline $0420-014$ & 0.915 & RLQ & 47.00 & -9.39201 & 9.03 & 4.3922 & 9.60 & 0.916 & -14.01 & -24.14 & -14.96 \\
\hline $0437+785$ & 0.454 & RLQ & 46.15 & -9.15201 & 8.79 & 4.1522 & 9.97 & 0.458 & -12.72 & -22.85 & -13.67 \\
\hline $0444+634$ & 0.781 & RLQ & 46.12 & -8.89201 & 8.53 & 3.8922 & 9.48 & 0.784 & -12.93 & -23.06 & -13.88 \\
\hline $0454-810$ & 0.444 & RLQ & 45.32 & -8.49201 & 8.13 & 3.4922 & 9.48 & 0.450 & -11.54 & -21.67 & -12.49 \\
\hline $0454+066$ & 0.405 & RLQ & 45.12 & -7.78201 & 7.42 & 2.7822 & 8.26 & 0.407 & -10.19 & -20.32 & -11.14 \\
\hline $0502+049$ & 0.954 & RLQ & 46.36 & -9.24201 & 8.88 & 4.2422 & 9.94 & 0.957 & -13.80 & -23.93 & -14.75 \\
\hline $0514-459$ & 0.194 & RLQ & 45.36 & -7.91201 & 7.55 & 2.9122 & 8.28 & 0.196 & -9.61 & -19.74 & -10.56 \\
\hline $0518+165$ & 0.759 & RLQ & 46.34 & -8.89201 & 8.53 & 3.8922 & 9.26 & 0.761 & -12.89 & -23.02 & -13.84 \\
\hline $0538+498$ & 0.545 & RLQ & 46.43 & -9.94201 & 9.58 & 4.9422 & 11.27 & 0.559 & -14.32 & -24.45 & -15.27 \\
\hline $0602-319$ & 0.452 & RLQ & 45.69 & -9.38201 & 9.02 & 4.3822 & 10.89 & 0.473 & -13.11 & -23.25 & -14.07 \\
\hline
\end{tabular}


TABle 3: Continued.

\begin{tabular}{|c|c|c|c|c|c|c|c|c|c|c|c|}
\hline Name & $z$ & Type & $\log L_{\text {bol }}$ & $\log \left(\frac{R_{d}}{r_{\mathrm{ov}}}\right)$ & $\log \left(\frac{M_{\mathrm{BH}}}{M_{\odot}}\right)$ & $\log \left(\frac{M_{\mathrm{BH}}^{\text {Seed }}}{M_{\odot}}\right)$ & $\log T_{\mathrm{BH}}$ & $z^{\text {Seed }}$ & $J^{q}$ & $J^{\mathrm{URCA}}$ & $J^{\pi}$ \\
\hline $0607-157$ & 0.324 & RLQ & 46.30 & -9.04201 & 8.68 & 4.0422 & 9.60 & 0.326 & -12.14 & -22.27 & -13.09 \\
\hline $0637-752$ & 0.654 & RLQ & 47.16 & -9.77201 & 9.41 & 4.7722 & 10.20 & 0.656 & -14.24 & -24.37 & -15.19 \\
\hline $0646+600$ & 0.455 & RLQ & 45.58 & -9.10201 & 8.74 & 4.1022 & 10.44 & 0.469 & -12.63 & -22.76 & -13.58 \\
\hline $0723+679$ & 0.846 & RLQ & 46.41 & -9.03201 & 8.67 & 4.0322 & 9.47 & 0.848 & -13.27 & -23.41 & -14.23 \\
\hline $0736+017$ & 0.191 & RLQ & 46.41 & -8.36201 & 8.00 & 3.3622 & 8.57 & 0.192 & -10.38 & -20.51 & -11.33 \\
\hline $0738+313$ & 0.631 & RLQ & 46.94 & -9.76201 & 9.40 & 4.7622 & 10.40 & 0.634 & -14.18 & -24.31 & -15.13 \\
\hline $0809+483$ & 0.871 & RLQ & 46.54 & -8.32201 & 7.96 & 3.3222 & 7.92 & 0.871 & -12.07 & -22.20 & -13.02 \\
\hline $0838+133$ & 0.684 & RLQ & 46.23 & -8.88201 & 8.52 & 3.8822 & 9.35 & 0.686 & -12.74 & -22.87 & -13.69 \\
\hline $0906+430$ & 0.668 & RLQ & 45.99 & -8.26201 & 7.90 & 3.2622 & 8.35 & 0.669 & -11.63 & -21.76 & -12.58 \\
\hline $0912+029$ & 0.427 & RLQ & 45.26 & -8.08201 & 7.72 & 3.0822 & 8.72 & 0.430 & -10.77 & -20.90 & -11.72 \\
\hline $0921-213$ & 0.052 & RLQ & 44.63 & -8.50201 & 8.14 & 3.5022 & 10.19 & 0.084 & -9.36 & -19.50 & -10.32 \\
\hline $0923+392$ & 0.698 & RLQ & 46.26 & -9.64201 & 9.28 & 4.6422 & 10.84 & 0.708 & -14.10 & -24.23 & -15.05 \\
\hline $0925-203$ & 0.348 & RLQ & 46.35 & -8.82201 & 8.46 & 3.8222 & 9.11 & 0.349 & -11.83 & -21.97 & -12.78 \\
\hline $0953+254$ & 0.712 & RLQ & 46.59 & -9.36201 & 9.00 & 4.3622 & 9.95 & 0.715 & -13.63 & -23.76 & -14.58 \\
\hline $0954+556$ & 0.901 & RLQ & 46.54 & -8.43201 & 8.07 & 3.4322 & 8.14 & 0.901 & -12.31 & -22.44 & -13.26 \\
\hline $1004+130$ & 0.240 & RLQ & 46.21 & -9.46201 & 9.10 & 4.4622 & 10.53 & 0.248 & -12.55 & -22.68 & -13.50 \\
\hline $1007+417$ & 0.612 & RLQ & 46.71 & -9.15201 & 8.79 & 4.1522 & 9.41 & 0.613 & -13.08 & -23.21 & -14.03 \\
\hline $1016-311$ & 0.794 & RLQ & 46.63 & -9.25201 & 8.89 & 4.2522 & 9.69 & 0.796 & -13.58 & -23.71 & -14.53 \\
\hline $1020-103$ & 0.197 & RLQ & 44.87 & -8.72201 & 8.36 & 3.7222 & 10.39 & 0.228 & -11.04 & -21.18 & -11.99 \\
\hline $1034-293$ & 0.312 & RLQ & 46.20 & -9.11201 & 8.75 & 4.1122 & 9.84 & 0.316 & -12.22 & -22.35 & -13.17 \\
\hline $1036-154$ & 0.525 & RLQ & 44.55 & -8.16201 & 7.80 & 3.1622 & 9.59 & 0.543 & -11.16 & -21.29 & -12.11 \\
\hline $1045-188$ & 0.595 & RLQ & 45.80 & -7.19201 & 6.83 & 2.1922 & 6.40 & 0.595 & -9.61 & -19.74 & -10.56 \\
\hline $1100+772$ & 0.311 & RLQ & 46.49 & -9.67201 & 9.31 & 4.6722 & 10.67 & 0.318 & -13.20 & -23.33 & -14.15 \\
\hline $1101-325$ & 0.355 & RLQ & 46.33 & -8.97201 & 8.61 & 3.9722 & 9.43 & 0.357 & -12.12 & -22.25 & -13.07 \\
\hline $1106+023$ & 0.157 & RLQ & 44.97 & -7.86201 & 7.50 & 2.8622 & 8.57 & 0.160 & -9.31 & -19.44 & -10.26 \\
\hline $1107-187$ & 0.497 & RLQ & 44.25 & -7.26201 & 6.90 & 2.2622 & 8.09 & 0.501 & -9.52 & -19.65 & -10.47 \\
\hline $1111+408$ & 0.734 & RLQ & 46.26 & -10.18201 & 9.82 & 5.1822 & 11.92 & 0.770 & -15.11 & -25.24 & -16.06 \\
\hline $1128-047$ & 0.266 & RLQ & 44.08 & -7.08201 & 6.72 & 2.0822 & 7.90 & 0.270 & -8.49 & -18.62 & -9.44 \\
\hline $1136-135$ & 0.554 & RLQ & 46.78 & -9.14201 & 8.78 & 4.1422 & 9.32 & 0.555 & -12.94 & -23.07 & -13.89 \\
\hline $1137+660$ & 0.656 & RLQ & 46.85 & -9.72201 & 9.36 & 4.7222 & 10.41 & 0.659 & -14.16 & -24.29 & -15.11 \\
\hline $1150+497$ & 0.334 & RLQ & 45.98 & -9.09201 & 8.73 & 4.0922 & 10.02 & 0.340 & -12.26 & -22.39 & -13.21 \\
\hline $1151-348$ & 0.258 & RLQ & 45.56 & -9.38201 & 9.02 & 4.3822 & 11.02 & 0.287 & -12.26 & -22.39 & -13.21 \\
\hline $1200-051$ & 0.381 & RLQ & 46.41 & -8.77201 & 8.41 & 3.7722 & 8.95 & 0.382 & -12.26 & -22.39 & -13.21 \\
\hline $1202-262$ & 0.789 & RLQ & 45.81 & -9.36201 & 9.00 & 4.3622 & 10.73 & 0.804 & -13.76 & -23.89 & -14.71 \\
\hline $1217+023$ & 0.240 & RLQ & 45.83 & -8.77201 & 8.41 & 3.7722 & 9.53 & 0.244 & -11.34 & -21.47 & -12.29 \\
\hline $1237-101$ & 0.751 & RLQ & 46.63 & -9.64201 & 9.28 & 4.6422 & 10.47 & 0.755 & -14.19 & -24.32 & -15.14 \\
\hline $1244-255$ & 0.633 & RLQ & 46.48 & -9.40201 & 9.04 & 4.4022 & 10.14 & 0.637 & -13.55 & -23.69 & -14.51 \\
\hline $1250+568$ & 0.321 & RLQ & 45.61 & -8.78201 & 8.42 & 3.7822 & 9.77 & 0.327 & -11.67 & -21.81 & -12.62 \\
\hline $1253-055$ & 0.536 & RLQ & 46.10 & -8.79201 & 8.43 & 3.7922 & 9.30 & 0.538 & -12.28 & -22.42 & -13.24 \\
\hline $1254-333$ & 0.190 & RLQ & 45.52 & -9.19201 & 8.83 & 4.1922 & 10.68 & 0.210 & -11.83 & -21.96 & -12.78 \\
\hline $1302-102$ & 0.286 & RLQ & 45.86 & -8.66201 & 8.30 & 3.6622 & 9.28 & 0.289 & -11.34 & -21.47 & -12.29 \\
\hline $1352-104$ & 0.332 & RLQ & 45.81 & -8.51201 & 8.15 & 3.5122 & 9.03 & 0.334 & -11.24 & -21.37 & -12.19 \\
\hline $1354+195$ & 0.720 & RLQ & 47.11 & -9.80201 & 9.44 & 4.8022 & 10.31 & 0.722 & -14.42 & -24.55 & -15.37 \\
\hline $1355-416$ & 0.313 & RLQ & 46.48 & -10.09201 & 9.73 & 5.0922 & 11.52 & 0.331 & -13.94 & -24.07 & -14.89 \\
\hline $1359-281$ & 0.803 & RLQ & 46.19 & -8.43201 & 8.07 & 3.4322 & 8.49 & 0.804 & -12.16 & -22.29 & -13.11 \\
\hline $1450-338$ & 0.368 & RLQ & 43.94 & -6.82201 & 6.46 & 1.8222 & 7.52 & 0.371 & -8.40 & -18.53 & -9.35 \\
\hline $1451-375$ & 0.314 & RLQ & 46.16 & -9.18201 & 8.82 & 4.1822 & 10.02 & 0.319 & -12.35 & -22.48 & -13.30 \\
\hline $1458+718$ & 0.905 & RLQ & 46.93 & -9.34201 & 8.98 & 4.3422 & 9.57 & 0.906 & -13.91 & -24.04 & -14.86 \\
\hline $1509+022$ & 0.219 & RLQ & 44.54 & -8.35201 & 7.99 & 3.3522 & 9.98 & 0.247 & -10.51 & -20.64 & -11.46 \\
\hline $1510-089$ & 0.361 & RLQ & 46.38 & -9.01201 & 8.65 & 4.0122 & 9.46 & 0.363 & -12.21 & -22.34 & -13.16 \\
\hline
\end{tabular}


TABLE 3: Continued.

\begin{tabular}{lcccccccccccc}
\hline Name & $z$ & Type & $\log L_{\text {bol }}$ & $\log \left(\frac{R_{d}}{r_{\mathrm{ov}}}\right)$ & $\log \left(\frac{M_{\mathrm{BH}}}{M_{\odot}}\right)$ & $\log \left(\frac{M_{\mathrm{BH}}^{\mathrm{Sed}}}{M_{\odot}}\right)$ & $\log T_{\mathrm{BH}}$ & $z^{\text {Seed }}$ & $J^{q}$ & $J^{\mathrm{URCA}}$ & $J^{\pi}$ \\
\hline $1545+210$ & 0.266 & RLQ & 45.86 & -9.29201 & 8.93 & 4.2922 & 10.54 & 0.278 & -12.36 & -22.49 & -13.31 \\
$1546+027$ & 0.412 & RLQ & 46.00 & -9.08201 & 8.72 & 4.0822 & 9.98 & 0.417 & -12.48 & -22.61 & -13.43 \\
$1555-140$ & 0.097 & RLQ & 44.94 & -7.61201 & 7.25 & 2.6122 & 8.10 & 0.099 & -8.39 & -18.53 & -9.34 \\
$1611+343$ & 1.401 & RLQ & 46.99 & -9.93201 & 9.57 & 4.9322 & 10.69 & 1.405 & -15.54 & -25.67 & -16.49 \\
$1634+628$ & 0.988 & RLQ & 45.47 & -7.64201 & 7.28 & 2.6422 & 7.63 & 0.989 & -11.05 & -21.18 & -12.00 \\
$1637+574$ & 0.750 & RLQ & 46.68 & -9.54201 & 9.18 & 4.5422 & 10.22 & 0.753 & -14.01 & -24.14 & -14.96 \\
$1641+399$ & 0.594 & RLQ & 46.89 & -9.78201 & 9.42 & 4.7822 & 10.49 & 0.597 & -14.14 & -24.27 & -15.09 \\
$1642+690$ & 0.751 & RLQ & 45.78 & -8.12201 & 7.76 & 3.1222 & 8.28 & 0.752 & -11.53 & -21.66 & -12.48 \\
$1656+053$ & 0.879 & RLQ & 47.21 & -9.98201 & 9.62 & 4.9822 & 10.57 & 0.882 & -14.99 & -25.12 & -15.94 \\
$1706+006$ & 0.449 & RLQ & 44.01 & -6.99210 & 6.63 & 2.9922 & 7.79 & 0.453 & -8.92 & -19.06 & -9.87 \\
$1721+343$ & 0.206 & RLQ & 45.63 & -8.40201 & 8.04 & 3.4022 & 8.99 & 0.209 & -10.53 & -20.66 & -11.48 \\
$1725+044$ & 0.293 & RLQ & 46.07 & -8.43201 & 8.07 & 3.4322 & 8.61 & 0.294 & -10.96 & -21.09 & -11.91 \\
\hline
\end{tabular}

spacetime deformation theory [82] by new investigation of building up the distortion-complex of spacetime continuum and showing how it restores the world-deformation tensor, which still has been put in by hand. We extend necessary geometrical ideas of spacetime deformation in concise form, without going into the subtleties, as applied to the gravitation theory which underlies the MTBH framework. I have attempted to maintain a balance between being overly detailed and overly schematic. Therefore the text in the appendices should resemble a "hybrid" of a new investigation and some issues of proposed gravitation theory.

A.1. A First Glance at Spacetime Deformation. Consider a smooth deformation map $\Omega: M_{4} \rightarrow \widetilde{\mathscr{M}}_{4}$, written in terms of the world-deformation tensor $(\Omega)$, the general $\left(\widetilde{M}_{4}\right)$, and flat $\left(M_{4}\right)$ smooth differential $4 \mathrm{D}$-manifolds. The following notational conventions will be used throughout the appendices. All magnitudes related to the space, $\widetilde{\mathscr{M}}_{4}$, will be denoted by an over " $\sim$ ". We use the Greek alphabet $(\mu, \nu, \rho, \ldots=0,1,2,3)$ to denote the holonomic world indices related to $\widetilde{\mathscr{M}}_{4}$ and the second half of Latin alphabet $(l, m, k, \ldots=0,1,2,3)$ to denote the world indices related to $M_{4}$. The tensor, $\Omega$, can be written in the form $\Omega=\widetilde{D} \widetilde{\psi}\left(\Omega^{m}{ }_{l}=\right.$ $\left.\widetilde{D}_{\mu}^{m} \widetilde{\psi}_{l}^{\mu}\right)$, where the DC-members are the invertible distortion matrix $\widetilde{D}\left(\widetilde{D}_{\mu}^{m}\right)$ and the tensor $\widetilde{\psi}\left(\widetilde{\psi}_{l}^{\mu} \equiv \partial_{l} \widetilde{x}^{\mu}\right.$ and $\left.\partial_{l}=\partial / \partial x^{l}\right)$. The principle foundation of the world-deformation tensor $(\Omega)$ comprises the following two steps: (1) the basis vectors $e_{m}$ at given point $\left(p \in M_{4}\right)$ undergo the distortion transformations by means of $\widetilde{D}$; and (2) the diffeomorphism $\widetilde{x}^{\mu}(x): M_{4} \rightarrow$ $\widetilde{M}_{4}$ is constructed by seeking new holonomic coordinates $\tilde{x}^{\mu}(x)$ as the solutions of the first-order partial differential equations. Namely,

$$
\widetilde{e}_{\mu}=\widetilde{D}_{\mu}^{l} e_{l}, \quad \widetilde{e}_{\mu} \widetilde{\psi}_{l}^{\mu}=\Omega^{m}{ }_{l} e_{m},
$$

where the conditions of integrability, $\partial_{k} \psi_{l}^{\mu}=\partial_{l} \psi_{k}^{\mu}$, and nondegeneracy, $\|\psi\| \neq 0$, necessarily hold [83, 84]. For reasons that will become clear in the sequel, next we write the norm $d \widetilde{s} \equiv i \widetilde{d}$ (see Appendix B) of the infinitesimal displacement $d \widetilde{x}^{\mu}$ on the $\widetilde{\mathscr{M}}_{4}$ in terms of the spacetime structures of $M_{4}$

$$
i \widetilde{d}=\widetilde{e} \widetilde{\vartheta}=\widetilde{e}_{\mu} \otimes \widetilde{\vartheta}^{\mu}=\Omega^{m}{ }_{l} e_{m} \otimes \vartheta^{l} \in \widetilde{M}_{4} .
$$

A deformation $\Omega: M_{4} \rightarrow \widetilde{M}_{4}$ comprises the following two $4 \mathrm{D}$ deformations $\stackrel{\circ}{\Omega}: M_{4} \rightarrow V_{4}$ and $\breve{\Omega}: V_{4} \rightarrow$ $\widetilde{M}_{4}$, where $V_{4}$ is the semi-Riemannian space and $\stackrel{\Omega}{\Omega}$ and $\breve{\Omega}$ are the corresponding world deformation tensors. The key points of the theory of spacetime deformation are outlined further in Appendix B. Finally, to complete this theory we need to determine $\widetilde{D}$ and $\widetilde{\psi}$, figured in (A.1). In the standard theory of gravitation they can be determined from the standard field equations by means of the general linear frames (C.10). However, it should be emphasized that the standard Riemannian space interacting quantum field theory cannot be a satisfactory ground for addressing the most important processes of rearrangement of vacuum state and gauge symmetry breaking in gravity at huge energies. The difficulties arise there because Riemannian geometry, in general, does not admit a group of isometries, and it is impossible to define energy-momentum as Noether local currents related to exact symmetries. This, in turn, posed severe problem of nonuniqueness of the physical vacuum and the associated Fock space. A definition of positive frequency modes cannot, in general, be unambiguously fixed in the past and future, which leads to $\mid$ in $\rangle \neq \mid$ out $\rangle$, because the state $\mid$ in $\rangle$ is unstable against decay into many particle $\mid$ out $\rangle$ states due to interaction processes allowed by lack of Poincaré invariance. A nontrivial Bogolubov transformation between past and future positive frequency modes implies that particles are created from the vacuum and this is one of the reasons for $\mid$ in $\rangle \neq \mid$ out $\rangle$.

A.2. General Gauge Principle. Keeping in mind the aforesaid, we develop the alternative framework of the general gauge principle (GGP), which is the distortion gauge induced fiberbundle formulation of gravitation. As this principle was in use as a guide in constructing our theory, we briefly discuss its general implications in Appendix D. The interested reader 
TABLE 4: Seed black hole intermediate masses, preradiation times, redshifts, and neutrino fluxes from observed stellar velocity dispersions. Columns: (1) name, (2) redshift, (3) AGN type: SY2: Seyfert 2, (4) log of the bolometric luminosity ( $\mathrm{ergs}^{-1}$ ), (5) log of the radius of protomatter core in special unit $r_{\mathrm{OV}}=13.68 \mathrm{~km},(6) \log$ of the black hole mass in solar masses, (7) $\log$ of the seed black hole intermediate mass in solar masses, (8) log of the neutrino preradiation time (yrs), (9) redshift of seed black hole, (10) $J^{i=q}$, (11) $J^{i=\mathrm{URCA}}$, and (12) $J^{i=\pi}$, where $J^{i} \equiv \log \left(J_{v \varepsilon}^{i} / \varepsilon_{d} \operatorname{erg~cm}^{-2} \mathrm{~s}^{-1} \mathrm{sr}^{-1}\right)$.

\begin{tabular}{|c|c|c|c|c|c|c|c|c|c|c|c|}
\hline Name & $z$ & Type & $\log L_{\text {bol }}$ & $\log \left(\frac{R_{d}}{r_{\mathrm{ov}}}\right)$ & $\log \left(\frac{M_{\mathrm{BH}}}{M_{\odot}}\right)$ & $\log \left(\frac{M_{\mathrm{BH}}^{\text {Seed }}}{M_{\odot}}\right)$ & $\log T_{\mathrm{BH}}$ & $z^{\text {Seed }}$ & $J^{q}$ & $J^{\mathrm{URCA}}$ & $J^{\pi}$ \\
\hline NGC 1566 & 0.005 & SY1 & 44.45 & -7.28201 & 6.92 & 2.2822 & 7.93 & 0.008 & -5.15 & -15.28 & -6.10 \\
\hline NGC 2841 & 0.002 & SY1 & 43.67 & -8.57201 & 8.21 & 3.5722 & 11.29 & 0.347 & -6.60 & -16.74 & -7.55 \\
\hline NGC 3982 & 0.004 & SY1 & 43.54 & -6.45201 & 6.09 & 1.45220 & 7.18 & 0.008 & -3.50 & -13.63 & -4.45 \\
\hline NGC 3998 & 0.003 & SY1 & 43.54 & -9.31201 & 8.95 & 4.3122 & 12.90 & 2.561 & -8.25 & -18.38 & -9.20 \\
\hline Mrk 10 & 0.029 & SY1 & 44.61 & -7.83291 & 7.47 & 4.7908 & 8.87 & 0.036 & -7.66 & -17.79 & -8.61 \\
\hline UGC 3223 & 0.016 & SY1 & 44.27 & -7.38201 & 7.02 & 2.3822 & 8.31 & 0.022 & -6.34 & -16.47 & -7.29 \\
\hline NGC 513 & 0.002 & SY2 & 42.52 & -8.01201 & 7.65 & 3.0122 & 11.32 & 1.345 & -5.62 & -15.76 & -6.57 \\
\hline NGC 788 & 0.014 & SY2 & 44.33 & -7.87201 & 7.51 & 2.8722 & 9.23 & 0.029 & -7.08 & -17.21 & -8.03 \\
\hline NGC 1052 & 0.005 & SY2 & 43.84 & -8.55201 & 8.19 & 3.5522 & 11.08 & 0.228 & -7.37 & -17.50 & -8.32 \\
\hline NGC 1275 & 0.018 & SY2 & 45.04 & -8.87201 & 8.51 & 3.8722 & 10.52 & 0.047 & -9.05 & -19.19 & -10.01 \\
\hline NGC 1320 & 0.009 & SY2 & 44.02 & -7.54201 & 7.18 & 2.5422 & 8.88 & 0.023 & -6.12 & -16.25 & -7.07 \\
\hline NGC 1358 & 0.013 & SY2 & 44.37 & -8.24201 & 7.88 & 3.2422 & 9.93 & 0.045 & -7.66 & -17.80 & -8.62 \\
\hline NGC 1386 & 0.003 & SY2 & 43.38 & -7.60201 & 7.24 & 2.6022 & 9.64 & 0.075 & -0.020 & -15.39 & -6.21 \\
\hline NGC 1667 & 0.015 & SY2 & 44.69 & -8.24201 & 7.88 & 3.2422 & 9.61 & 0.030 & -7.79 & -17.92 & -8.74 \\
\hline NGC 2110 & 0.008 & SY2 & 44.10 & -8.66201 & 8.30 & 3.6622 & 11.04 & 0.166 & -7.97 & -18.10 & -8.92 \\
\hline NGC 2273 & 0.006 & SY2 & 44.05 & -7.66201 & 7.30 & 2.6622 & 9.09 & 0.024 & -5.97 & -16.10 & -6.92 \\
\hline NGC 2992 & 0.008 & SY2 & 43.92 & -8.08201 & 7.72 & 3.0822 & 10.06 & 0.071 & -6.96 & -17.09 & -7.91 \\
\hline NGC 3185 & 0.004 & SY2 & 43.08 & -6.42201 & 6.06 & 1.4222 & 7.58 & 0.014 & -3.45 & -13.58 & -4.40 \\
\hline NGC 3362 & 0.028 & SY2 & 44.27 & -7.13201 & 6.77 & 2.1322 & 7.81 & 0.031 & -6.40 & -16.54 & -7.36 \\
\hline NGC 3786 & 0.009 & SY2 & 43.47 & -7.89201 & 7.53 & 2.8922 & 10.13 & 0.123 & -6.73 & -16.86 & -7.68 \\
\hline NGC 4117 & 0.003 & SY2 & 43.64 & -7.19201 & 6.83 & 2.1922 & 8.56 & 0.018 & -4.54 & -14.67 & -5.49 \\
\hline NGC 4339 & 0.004 & SY2 & 43.38 & -7.76201 & 7.40 & 2.7622 & 9.96 & 0.108 & -5.79 & -15.92 & -6.74 \\
\hline NGC 5194 & 0.002 & SY2 & 43.79 & -7.31201 & 6.95 & 2.3122 & 8.65 & 0.016 & -4.40 & -14.53 & -5.35 \\
\hline NGC 5252 & 0.023 & SY2 & 45.39 & -8.40201 & 8.04 & 3.4022 & 9.23 & 0.027 & -8.45 & -18.58 & -9.40 \\
\hline NGC 5273 & 0.004 & SY2 & 43.03 & -6.87201 & 6.51 & 1.8722 & 8.53 & 0.034 & -4.23 & -14.36 & -5.18 \\
\hline NGC 5347 & 0.008 & SY2 & 43.81 & -7.15201 & 6.79 & 2.1522 & 8.31 & 0.018 & -5.33 & -15.46 & -6.28 \\
\hline NGC 5427 & 0.009 & SY2 & 44.12 & -6.75201 & 6.39 & 1.7522 & 7.20 & 0.011 & -4.73 & -14.86 & -5.68 \\
\hline NGC 5929 & 0.008 & SY2 & 43.04 & -7.61201 & 7.25 & 2.6122 & 10.00 & 0.169 & -6.13 & -16.27 & -7.09 \\
\hline NGC 5953 & 0.007 & SY2 & 44.05 & -7.30201 & 6.94 & 2.3022 & 8.37 & 0.015 & -5.48 & -15.61 & -6.43 \\
\hline NGC 6104 & 0.028 & SY2 & 43.60 & -7.96201 & 7.60 & 2.9622 & 10.14 & 0.128 & -7.86 & -17.99 & -8.81 \\
\hline NGC 7213 & 0.006 & SY2 & 44.30 & -8.35201 & 7.99 & 3.3522 & 10.22 & 0.055 & -7.18 & -17.31 & -8.13 \\
\hline NGC 7319 & 0.023 & SY2 & 44.19 & -7.74201 & 7.38 & 2.7422 & 9.11 & 0.038 & -7.30 & -17.43 & -8.25 \\
\hline NGC 7603 & 0.030 & SY2 & 44.66 & -8.44201 & 8.08 & 3.4422 & 10.04 & 0.056 & -8.76 & -18.89 & -9.71 \\
\hline NGC 7672 & 0.013 & SY2 & 43.86 & -7.24201 & 6.88 & 2.2422 & 8.44 & 0.023 & -5.91 & -16.05 & -6.87 \\
\hline NGC 7682 & 0.017 & SY2 & 43.93 & -7.64201 & 7.28 & 2.6422 & 9.17 & 0.039 & -6.85 & -16.98 & -7.80 \\
\hline NGC 7743 & 0.006 & SY2 & 43.60 & -6.95201 & 6.59 & 1.9522 & 8.12 & 0.016 & -4.73 & -14.86 & -5.68 \\
\hline Mrk 1 & 0.016 & SY2 & 44.20 & -7.52201 & 7.16 & 2.5222 & 8.66 & 0.025 & -6.59 & -16.72 & -7.54 \\
\hline Mrk 3 & 0.014 & SY2 & 44.54 & -9.01201 & 8.65 & 4.0122 & 11.30 & 0.142 & -9.08 & -19.21 & -10.03 \\
\hline Mrk 78 & 0.037 & SY2 & 44.59 & -8.23201 & 7.87 & 3.2322 & 9.69 & 0.056 & -8.58 & -18.71 & -9.53 \\
\hline Mrk 270 & 0.010 & SY2 & 43.37 & -7.96201 & 7.60 & 2.9622 & 10.37 & 0.179 & -6.94 & -17.07 & -7.89 \\
\hline Mrk 348 & 0.015 & SY2 & 44.27 & -7.57201 & 7.21 & 2.5722 & 8.69 & 0.024 & -6.62 & -16.75 & -7.57 \\
\hline Mrk 533 & 0.029 & SY2 & 45.15 & -7.92201 & 7.56 & 2.9222 & 8.51 & 0.032 & -7.82 & -17.95 & -8.77 \\
\hline Mrk 573 & 0.017 & SY2 & 44.44 & -7.64201 & 7.28 & 2.6422 & 8.66 & 0.024 & -6.85 & -16.98 & -7.80 \\
\hline Mrk 622 & 0.023 & SY2 & 44.52 & -7.28201 & 6.92 & 2.2822 & 7.86 & 0.026 & -6.49 & -16.62 & -7.44 \\
\hline Mrk 686 & 0.014 & SY2 & 44.11 & -7.92201 & 7.56 & 2.9222 & 9.55 & 0.042 & -7.17 & -17.30 & -8.12 \\
\hline Mrk 917 & 0.024 & SY2 & 44.75 & -7.98201 & 7.62 & 2.9822 & 9.03 & 0.031 & -7.75 & -17.89 & -8.70 \\
\hline Mrk 1018 & 0.042 & SY2 & 44.39 & -8.45201 & 8.09 & 3.4522 & 10.33 & 0.092 & -9.08 & -19.21 & -10.03 \\
\hline
\end{tabular}


TABle 4: Continued.

\begin{tabular}{|c|c|c|c|c|c|c|c|c|c|c|c|}
\hline Name & $z$ & Type & $\log L_{\text {bol }}$ & $\log \left(\frac{R_{d}}{r_{\mathrm{ov}}}\right)$ & $\log \left(\frac{M_{\mathrm{BH}}}{M_{\odot}}\right)$ & $\log \left(\frac{M_{\mathrm{BH}}^{\text {Seed }}}{M_{\odot}}\right)$ & $\log T_{\mathrm{BH}}$ & $z^{\text {Seed }}$ & $J^{q}$ & $J^{\mathrm{URCA}}$ & $J^{\pi}$ \\
\hline Mrk 1040 & 0.017 & SY2 & 44.53 & -8.00201 & 7.64 & 3.0022 & 9.29 & 0.030 & -7.48 & -17.61 & -8.43 \\
\hline Mrk 1066 & 0.012 & SY2 & 44.55 & -7.37201 & 7.01 & 2.3722 & 8.01 & 0.015 & -6.07 & -16.20 & -7.02 \\
\hline Mrk 1157 & 0.015 & SY2 & 44.27 & -7.19201 & 6.83 & 2.1922 & 7.93 & 0.019 & -5.95 & -16.08 & -6.90 \\
\hline Akn 79 & 0.018 & SY2 & 45.24 & -7.90201 & 7.54 & 2.9022 & 8.38 & 0.020 & -7.36 & -17.49 & -8.31 \\
\hline Akn 347 & 0.023 & SY2 & 44.84 & -8.36201 & 8.00 & 3.3622 & 9.70 & 0.037 & -8.38 & -18.51 & -9.33 \\
\hline IC 5063 & 0.011 & SY2 & 44.53 & -8.10201 & 7.74 & 3.1022 & 9.49 & 0.027 & -7.27 & -17.40 & -8.22 \\
\hline II ZW55 & 0.025 & SY2 & 44.54 & -8.59201 & 8.23 & 3.5922 & 10.46 & 0.074 & -8.86 & -18.99 & -9.81 \\
\hline F 341 & 0.016 & SY2 & 44.13 & -7.51201 & 7.15 & 2.5122 & 8.71 & 0.026 & -6.57 & -16.70 & -7.52 \\
\hline UGC 3995 & 0.016 & SY2 & 44.39 & -8.05201 & 7.69 & 3.0522 & 9.53 & 0.036 & -7.52 & -17.65 & -8.47 \\
\hline UGC 6100 & 0.029 & SY2 & 44.48 & -8.06201 & 7.70 & 3.0622 & 9.46 & 0.046 & -8.06 & -18.20 & -9.01 \\
\hline 1ES $1959+65$ & 0.048 & BLL & - & -10.39501 & 8.09 & 3.4522 & 7.79 & 0.052 & -9.20 & -19.34 & -10.15 \\
\hline Mrk 180 & 0.045 & BLL & - & -10.51501 & 8.21 & 3.5722 & 7.91 & 0.051 & -9.35 & -19.49 & -10.31 \\
\hline Mrk 421 & 0.031 & BLL & - & -10.59501 & 8.29 & 3.6522 & 7.99 & 0.038 & -9.16 & -19.29 & -10.11 \\
\hline Mrk 501 & 0.034 & BLL & - & -11.51501 & 9.21 & 4.5722 & 8.91 & 0.092 & -10.85 & -20.98 & -11.80 \\
\hline I Zw 187 & 0.055 & BLL & - & -10.16501 & 7.86 & 3.2222 & 7.56 & 0.058 & -8.93 & -19.06 & -9.88 \\
\hline 3C 371 & 0.051 & BLL & - & -10.81501 & 8.51 & 3.8722 & 8.21 & 0.063 & -9.99 & -20.13 & -10.95 \\
\hline $1514-241$ & 0.049 & BLL & - & -10.40501 & 8.10 & 3.4622 & 7.80 & 0.054 & -9.24 & -19.37 & -10.19 \\
\hline $0521-365$ & 0.055 & BLL & - & -10.95501 & 8.65 & 4.0122 & 8.35 & 0.071 & -10.31 & -20.44 & -11.26 \\
\hline $0548-322$ & 0.069 & BLL & - & -10.45501 & 8.15 & 3.5122 & 7.85 & 0.074 & -9.65 & -19.78 & -10.60 \\
\hline $0706+591$ & 0.125 & BLL & - & -10.56501 & 8.26 & 3.6222 & 7.96 & 0.132 & -10.41 & -20.54 & -11.36 \\
\hline $2201+044$ & 0.027 & BLL & - & -10.40501 & 8.10 & 3.4622 & 7.80 & 0.032 & -8.70 & -18.83 & -9.65 \\
\hline $2344+514$ & 0.044 & BLL & - & -11.10501 & 8.80 & 4.1622 & 8.50 & 0.067 & -10.37 & -20.50 & -11.32 \\
\hline $3 \mathrm{C} 29$ & 0.045 & $\mathrm{RG}$ & - & -10.50501 & 8.20 & 3.5622 & 7.90 & 0.051 & -9.34 & -19.47 & -10.29 \\
\hline 3C 31 & 0.017 & RG & - & -10.80501 & 8.50 & 3.8622 & 8.20 & 0.028 & -8.99 & -19.12 & -9.94 \\
\hline $3 C 33$ & 0.059 & RG & - & -10.68501 & 8.38 & 3.7422 & 8.08 & 0.068 & -9.90 & -20.03 & -10.85 \\
\hline $3 \mathrm{C} 40$ & 0.018 & RG & - & -10.16501 & 7.86 & 3.2222 & 7.56 & 0.021 & -7.92 & -18.05 & -8.87 \\
\hline $3 \mathrm{C} 62$ & 0.148 & RG & - & -10.97501 & 8.67 & 4.0322 & 8.37 & 0.165 & -11.29 & -21.43 & -12.25 \\
\hline 3C 76.1 & 0.032 & $\mathrm{RG}$ & - & -10.43501 & 8.13 & 3.4922 & 7.83 & 0.037 & -8.90 & -19.04 & -9.86 \\
\hline 3C 78 & 0.029 & $\mathrm{RG}$ & - & -10.90501 & 8.60 & 3.9622 & 8.30 & 0.043 & -9.64 & -19.77 & -10.59 \\
\hline $3 C 84$ & 0.017 & $\mathrm{RG}$ & - & -10.79501 & 8.49 & 3.8522 & 8.19 & 0.028 & -8.97 & -19.10 & -9.92 \\
\hline $3 C 88$ & 0.030 & $\mathrm{RG}$ & - & -10.33501 & 8.03 & 3.3922 & 7.73 & 0.034 & -8.67 & -18.80 & -9.62 \\
\hline $3 C 89$ & 0.139 & $\mathrm{RG}$ & - & -10.82501 & 8.52 & 3.8822 & 8.22 & 0.151 & -10.97 & -21.10 & -11.92 \\
\hline $3 \mathrm{C} 98$ & 0.031 & RG & - & -10.18501 & 7.88 & 3.2422 & 7.58 & 0.034 & -8.44 & -18.57 & -9.39 \\
\hline 3C 120 & 0.033 & $\mathrm{RG}$ & - & -10.43501 & 8.13 & 3.4922 & 7.83 & 0.038 & -8.93 & -19.06 & -9.88 \\
\hline 3C 192 & 0.060 & $\mathrm{RG}$ & - & -10.36501 & 8.06 & 3.4222 & 7.76 & 0.064 & -9.36 & -19.49 & -10.31 \\
\hline 3C 196.1 & 0.198 & $\mathrm{RG}$ & - & -10.51501 & 8.21 & 3.5722 & 7.91 & 0.204 & -10.79 & -20.92 & -11.74 \\
\hline $3 C 223$ & 0.137 & $\mathrm{RG}$ & - & -10.45501 & 8.15 & 3.5122 & 7.85 & 0.142 & -10.31 & -20.44 & -11.26 \\
\hline $3 C 293$ & 0.045 & $\mathrm{RG}$ & - & -10.29501 & 7.99 & 3.3522 & 7.69 & 0.048 & -8.97 & -19.10 & -9.92 \\
\hline 3C 305 & 0.041 & $\mathrm{RG}$ & - & -10.22501 & 7.92 & 3.2822 & 7.62 & 0.044 & -8.76 & -18.89 & -9.71 \\
\hline 3C 338 & 0.030 & $\mathrm{RG}$ & - & -11.08501 & 8.78 & 4.1422 & 8.48 & 0.052 & -9.98 & -20.12 & -10.93 \\
\hline 3C 388 & 0.091 & $\mathrm{RG}$ & - & -11.48501 & 9.18 & 4.5422 & 8.88 & 0.145 & -11.71 & -21.84 & -12.66 \\
\hline 3C 444 & 0.153 & $\mathrm{RG}$ & - & -9.98501 & 7.68 & 3.0422 & 7.38 & 0.155 & -9.60 & -19.73 & -10.55 \\
\hline 3C 449 & 0.017 & $\mathrm{RG}$ & - & -10.63501 & 8.33 & 3.6922 & 8.03 & 0.025 & -8.69 & -18.82 & -9.64 \\
\hline gin 116 & 0.033 & $\mathrm{RG}$ & - & -11.05501 & 8.75 & 4.1122 & 8.45 & 0.053 & -10.02 & -20.15 & -10.97 \\
\hline NGC 315 & 0.017 & RG & - & -11.20501 & 8.90 & 4.2622 & 8.60 & 0.045 & -9.69 & -19.82 & -10.64 \\
\hline NGC 507 & 0.017 & $\mathrm{RG}$ & - & -11.30501 & 9.00 & 4.3622 & 8.70 & 0.053 & -9.86 & -19.99 & -10.81 \\
\hline NGC 708 & 0.016 & $\mathrm{RG}$ & - & -10.76501 & 8.46 & 3.8222 & 8.16 & 0.026 & -8.86 & -18.99 & -9.81 \\
\hline NGC 741 & 0.018 & $\mathrm{RG}$ & - & -11.02501 & 8.72 & 4.0822 & 8.42 & 0.037 & -9.42 & -19.55 & -10.37 \\
\hline NGC 4839 & 0.023 & RG & - & -10.78501 & 8.48 & 3.8422 & 8.18 & 0.034 & -9.22 & -19.35 & -10.17 \\
\hline NGC 4869 & 0.023 & RG & - & -10.42501 & 8.12 & 3.4822 & 7.82 & 0.028 & -8.59 & -18.72 & -9.54 \\
\hline
\end{tabular}


TABle 4: Continued.

\begin{tabular}{|c|c|c|c|c|c|c|c|c|c|c|c|}
\hline Name & $z$ & Type & $\log L_{\text {bol }}$ & $\log \left(\frac{R_{d}}{r_{\mathrm{ov}}}\right)$ & $\log \left(\frac{M_{\mathrm{BH}}}{M_{\odot}}\right)$ & $\log \left(\frac{M_{\mathrm{BH}}^{\text {Seed }}}{M_{\odot}}\right)$ & $\log T_{\mathrm{BH}}$ & $z^{\text {Seed }}$ & $J^{q}$ & $J^{\mathrm{URCA}}$ & $J^{\pi}$ \\
\hline NGC 4874 & 0.024 & RG & - & -10.93501 & 8.63 & 3.9922 & 8.33 & 0.039 & -9.52 & -19.65 & -10.47 \\
\hline NGC 6086 & 0.032 & RG & - & -11.26501 & 8.96 & 4.3222 & 8.66 & 0.065 & -10.36 & -20.49 & -11.31 \\
\hline NGC 6137 & 0.031 & RG & - & -11.11501 & 8.81 & 4.1722 & 8.51 & 0.054 & -10.07 & -20.20 & -11.02 \\
\hline NGC 7626 & 0.025 & RG & - & -11.27501 & 8.97 & 4.3322 & 8.67 & 0.058 & -10.15 & -20.28 & -11.10 \\
\hline $0039-095$ & 0.000 & RG & - & -11.02501 & 8.72 & 4.0822 & 8.42 & 0.019 & -2.89 & -13.02 & -3.84 \\
\hline $0053-015$ & 0.038 & RG & - & -11.12501 & 8.82 & 4.1822 & 8.52 & 0.062 & -10.27 & -20.40 & -11.22 \\
\hline $0053-016$ & 0.043 & RG & - & -10.81501 & 8.51 & 3.8722 & 8.21 & 0.055 & -9.84 & -19.97 & -10.79 \\
\hline $0055-016$ & 0.045 & RG & - & -11.15501 & 8.85 & 4.2122 & 8.55 & 0.070 & -10.47 & -20.61 & -11.43 \\
\hline $0110+152$ & 0.044 & RG & - & -10.39501 & 8.09 & 3.4522 & 7.79 & 0.048 & -9.12 & -19.26 & -10.07 \\
\hline $0112-000$ & 0.045 & RG & - & -10.83501 & 8.53 & 3.8922 & 8.23 & 0.057 & -9.91 & -20.05 & -10.87 \\
\hline $0112+084$ & 0.000 & RG & - & -11.48501 & 9.18 & 4.5422 & 8.88 & 0.054 & -3.70 & -13.83 & -4.65 \\
\hline $0147+360$ & 0.018 & RG & - & -10.76501 & 8.46 & 3.8222 & 8.16 & 0.028 & -3.70 & -13.83 & -4.65 \\
\hline $0131-360$ & 0.030 & RG & - & -10.83501 & 8.53 & 3.8922 & 8.23 & 0.042 & -9.55 & -19.68 & -10.50 \\
\hline $0257-398$ & 0.066 & RG & - & -10.59501 & 8.29 & 3.6522 & 7.99 & 0.073 & -9.85 & -19.98 & -10.80 \\
\hline $0306+237$ & 0.000 & RG & - & -10.81501 & 8.51 & 3.8722 & 8.21 & 0.012 & -2.52 & -12.66 & -3.48 \\
\hline $0312-343$ & 0.067 & RG & - & -10.87501 & 8.57 & 3.9322 & 8.27 & 0.080 & -10.35 & -20.48 & -11.30 \\
\hline $0325+024$ & 0.030 & RG & - & -10.59501 & 8.29 & 3.6522 & 7.99 & 0.037 & -9.13 & -19.26 & -10.08 \\
\hline $0431-133$ & 0.033 & RG & - & -10.95501 & 8.65 & 4.0122 & 8.35 & 0.049 & -9.84 & -19.97 & -10.79 \\
\hline $0431-134$ & 0.035 & RG & - & -10.61501 & 8.31 & 3.6722 & 8.01 & 0.042 & -9.30 & -19.43 & -10.25 \\
\hline $0449-175$ & 0.031 & RG & - & -10.02501 & 7.72 & 3.0822 & 7.42 & 0.033 & -8.16 & -18.29 & -9.11 \\
\hline $0546-329$ & 0.037 & RG & - & -11.59501 & 9.29 & 4.6522 & 8.99 & 0.107 & -11.07 & -21.20 & -12.02 \\
\hline $0548-317$ & 0.034 & RG & - & -9.58501 & 7.28 & 2.6422 & 6.98 & 0.035 & -7.47 & -17.60 & -8.42 \\
\hline $0634-206$ & 0.056 & RG & - & -10.39501 & 8.09 & 3.4522 & 7.79 & 0.060 & -9.35 & -19.48 & -10.30 \\
\hline $0718-340$ & 0.029 & RG & - & -11.31501 & 9.01 & 4.3722 & 8.71 & 0.066 & -10.36 & -20.49 & -11.31 \\
\hline $0915-118$ & 0.054 & RG & - & -10.99501 & 8.69 & 4.0522 & 8.39 & 0.072 & -10.36 & -20.49 & -11.31 \\
\hline $0940-304$ & 0.038 & RG & - & -11.59501 & 9.29 & 4.6522 & 8.99 & 0.108 & -11.09 & -21.22 & -12.04 \\
\hline $1043-290$ & 0.060 & RG & - & -10.67501 & 8.37 & 3.7322 & 8.07 & 0.068 & -9.90 & -20.03 & -10.85 \\
\hline $1107-372$ & 0.010 & RG & - & -11.11501 & 8.81 & 4.1722 & 8.51 & 0.033 & -9.06 & -19.19 & -10.01 \\
\hline $1123-351$ & 0.032 & RG & - & -11.83501 & 9.53 & 4.8922 & 9.23 & 0.153 & -11.35 & -21.49 & -12.31 \\
\hline $1258-321$ & 0.015 & RG & - & -10.91501 & 8.61 & 3.9722 & 8.31 & 0.030 & -9.07 & -19.20 & -10.02 \\
\hline $1333-337$ & 0.013 & RG & - & -11.07501 & 8.77 & 4.1322 & 8.47 & 0.034 & -9.22 & -19.35 & -10.17 \\
\hline $1400-337$ & 0.014 & RG & - & -11.19501 & 8.89 & 4.2522 & 8.59 & 0.042 & -9.50 & -19.63 & -10.45 \\
\hline $1404-267$ & 0.022 & RG & - & -11.11505 & 8.81 & 4.8798 & 8.51 & 0.045 & -9.76 & -19.89 & -10.71 \\
\hline $1510+076$ & 0.053 & RG & - & -11.33501 & 9.03 & 4.3922 & 8.73 & 0.091 & -10.94 & -21.07 & -11.89 \\
\hline $1514+072$ & 0.035 & RG & - & -10.95501 & 8.65 & 4.0122 & 8.35 & 0.051 & -9.90 & -20.03 & -10.85 \\
\hline $1520+087$ & 0.034 & RG & - & -10.59501 & 8.29 & 3.6522 & 7.99 & 0.041 & -9.24 & -19.37 & -10.19 \\
\hline $1521-300$ & 0.020 & RG & - & -10.10501 & 7.80 & 3.1622 & 7.50 & 0.022 & -7.91 & -18.04 & -8.86 \\
\hline $1602+178$ & 0.041 & RG & - & -10.54501 & 8.24 & 3.6022 & 7.94 & 0.047 & -9.32 & -19.45 & -10.27 \\
\hline $1610+296$ & 0.032 & RG & - & -11.26501 & 8.96 & 4.3222 & 8.66 & 0.065 & -10.36 & -20.49 & -11.31 \\
\hline $2236-176$ & 0.070 & RG & - & -10.79501 & 8.49 & 3.8522 & 8.19 & 0.081 & -10.25 & -20.39 & -11.20 \\
\hline $2322+143$ & 0.045 & RG & - & -10.47501 & 8.17 & 3.5322 & 7.87 & 0.050 & -9.28 & -19.42 & -10.24 \\
\hline $2322-122$ & 0.082 & RG & - & -10.63501 & 8.33 & 3.6922 & 8.03 & 0.090 & -10.12 & -20.25 & -11.07 \\
\hline $2333-327$ & 0.052 & $\mathrm{RG}$ & - & -10.95501 & 8.65 & 4.0122 & 8.35 & 0.068 & -10.26 & -20.39 & -11.21 \\
\hline $2335+267$ & 0.030 & RG & - & -11.38501 & 9.08 & 4.4422 & 8.78 & 0.073 & -10.51 & -20.64 & -11.46 \\
\hline
\end{tabular}

is invited to consult the original paper [74] for details. In this, we restrict ourselves to consider only the simplest spacetime deformation map, $\widetilde{\Omega}: M_{4} \rightarrow V_{4}\left(\breve{\Omega}^{\mu}{ }_{\nu} \equiv\right.$ $\left.\delta_{v}^{\mu}\right)$. This theory accounts for the gravitation gauge group $G_{V}$ generated by the hidden local internal symmetry $U^{\text {loc }}$.
We assume that a distortion massless gauge field $a(x)(\equiv$ $\left.a_{n}(x)\right)$ has to act on the external spacetime groups. This field takes values in the Lie algebra of the abelian group $U^{\text {loc }}$. We pursue a principle goal of building up the worlddeformation tensor, $\widetilde{\Omega}(F)=\widetilde{D}(a) \widetilde{\psi}(a)$, where $F$ is the 
TABLE 5: Seed black hole intermediate masses, preradiation times, redshifts, and neutrino fluxes from fundamental plane-derived velocity dispersions. Columns: (1) name, (2) redshift, (3) AGN type: SY2: Seyfert 2, (4) log of the bolometric luminosity (ergs ${ }^{-1}$ ), (5) log of the radius of protomatter core in special unit $r_{\mathrm{OV}}=13.68 \mathrm{~km}$, (6) log of the black hole mass in solar masses, (7) $\log$ of the seed black hole intermediate mass in solar masses, (8) log of the neutrino preradiation time (yrs), (9) redshift of seed black hole, (10) $J^{i=q}$, (11) $J^{i=\mathrm{URCA}}$, and (12) $J^{i=\pi}$, where $J^{i} \equiv \log \left(J_{v \varepsilon}^{i} / \varepsilon_{d} \operatorname{erg~cm}^{-2} \mathrm{~s}^{-1} \mathrm{sr}^{-1}\right)$.

\begin{tabular}{|c|c|c|c|c|c|c|c|c|c|c|}
\hline Name & $z$ & Type & $\log \left(\frac{R_{d}}{r_{\mathrm{ov}}}\right)$ & $\log \left(\frac{M_{\mathrm{BH}}}{M_{\odot}}\right)$ & $\log \left(\frac{M_{\mathrm{BH}}^{\text {Seed }}}{M_{\odot}}\right)$ & $\log T_{\mathrm{BH}}$ & $z^{\text {Seed }}$ & $J^{q}$ & $J^{\mathrm{URCA}}$ & $J^{\pi}$ \\
\hline $0122+090$ & 0.339 & BLL & -11.12501 & 8.82 & 4.1822 & 8.52 & 0.363 & -12.43 & -22.57 & -13.39 \\
\hline $0145+138$ & 0.124 & BLL & -10.72501 & 8.42 & 3.7822 & 8.12 & 0.133 & -10.68 & -20.81 & -11.63 \\
\hline $0158+001$ & 0.229 & BLL & -10.38501 & 8.08 & 3.4422 & 7.78 & 0.233 & -10.71 & -20.84 & -11.66 \\
\hline $0229+200$ & 0.139 & BLL & -11.54501 & 9.24 & 4.6022 & 8.94 & 0.201 & -12.23 & -22.36 & -13.18 \\
\hline $0257+342$ & 0.247 & BLL & -10.96501 & 8.66 & 4.0222 & 8.36 & 0.263 & -11.81 & -21.94 & -12.76 \\
\hline $0317+183$ & 0.190 & BLL & -10.25501 & 7.95 & 3.3122 & 7.65 & 0.193 & -10.29 & -20.42 & -11.24 \\
\hline $0331-362$ & 0.308 & BLL & -11.05501 & 8.75 & 4.1122 & 8.45 & 0.328 & -12.21 & -22.34 & -13.16 \\
\hline $0347-121$ & 0.188 & BLL & -10.95501 & 8.65 & 4.0122 & 8.35 & 0.204 & -11.50 & -21.63 & -12.45 \\
\hline $0350-371$ & 0.165 & BLL & -11.12501 & 8.82 & 4.1822 & 8.52 & 0.189 & -11.67 & -21.80 & -12.62 \\
\hline $0414+009$ & 0.287 & BLL & -10.86501 & 8.56 & 3.9222 & 8.26 & 0.300 & -11.80 & -21.93 & -12.75 \\
\hline $0419+194$ & 0.512 & BLL & -10.91501 & 8.61 & 3.9722 & 8.31 & 0.527 & -12.54 & -22.68 & -13.50 \\
\hline $0506-039$ & 0.304 & BLL & -11.05501 & 8.75 & 4.1122 & 8.45 & 0.324 & -12.19 & -22.32 & -13.14 \\
\hline $0525+713$ & 0.249 & BLL & -11.33501 & 9.03 & 4.3922 & 8.73 & 0.287 & -12.46 & -22.60 & -13.41 \\
\hline $0607+710$ & 0.267 & BLL & -10.95501 & 8.65 & 4.0122 & 8.35 & 0.283 & -12.46 & -22.60 & -13.41 \\
\hline $0737+744$ & 0.315 & BLL & -11.24501 & 8.94 & 4.3022 & 8.64 & 0.346 & -12.56 & -22.69 & -13.51 \\
\hline $0922+749$ & 0.638 & BLL & -11.91501 & 9.61 & 4.9722 & 9.31 & 0.784 & -14.56 & -24.69 & -15.51 \\
\hline $0927+500$ & 0.188 & BLL & -10.64501 & 8.34 & 3.7022 & 8.04 & 0.196 & -10.96 & -21.09 & -11.91 \\
\hline $0958+210$ & 0.344 & BLL & -11.33501 & 9.03 & 4.3922 & 8.73 & 0.382 & -12.82 & -22.95 & -13.77 \\
\hline $1104+384$ & 0.031 & BLL & -11.69501 & 9.39 & 4.7522 & 9.09 & 0.119 & -11.08 & -21.21 & -12.03 \\
\hline $1133+161$ & 0.460 & BLL & -10.62501 & 8.32 & 3.6822 & 8.02 & 0.467 & -11.91 & -22.04 & -12.86 \\
\hline $1136+704$ & 0.045 & BLL & -11.25501 & 8.95 & 4.3122 & 8.65 & 0.077 & -10.65 & -20.78 & -11.60 \\
\hline $1207+394$ & 0.615 & BLL & -11.40501 & 9.10 & 4.4622 & 8.80 & 0.660 & -13.62 & -23.76 & -14.58 \\
\hline $1212+078$ & 0.136 & BLL & -11.29501 & 8.99 & 4.3522 & 8.69 & 0.171 & -11.77 & -21.90 & -12.72 \\
\hline $1215+303$ & 0.130 & BLL & -10.42501 & 8.12 & 3.4822 & 7.82 & 0.135 & -10.20 & -20.33 & -11.15 \\
\hline $1218+304$ & 0.182 & BLL & -10.88501 & 8.58 & 3.9422 & 8.28 & 0.196 & -11.35 & -21.48 & -12.30 \\
\hline $1221+245$ & 0.218 & BLL & -10.27501 & 7.97 & 3.3322 & 7.67 & 0.221 & -10.47 & -20.60 & -11.42 \\
\hline $1229+643$ & 0.164 & BLL & -11.71501 & 9.41 & 4.7722 & 9.11 & 0.256 & -12.69 & -22.82 & -13.64 \\
\hline $1248-296$ & 0.370 & BLL & -11.31501 & 9.01 & 4.3722 & 8.71 & 0.407 & -12.87 & -23.00 & -13.82 \\
\hline $1255+244$ & 0.141 & BLL & -10.88501 & 8.58 & 3.9422 & 8.28 & 0.155 & -11.09 & -21.22 & -12.04 \\
\hline $1407+595$ & 0.495 & BLL & -11.60501 & 9.30 & 4.6622 & 9.00 & 0.566 & -13.71 & -23.84 & -14.66 \\
\hline $1418+546$ & 0.152 & BLL & -11.33501 & 9.03 & 4.3922 & 8.73 & 0.190 & -11.95 & -22.08 & -12.90 \\
\hline $1426+428$ & 0.129 & BLL & -11.43501 & 9.13 & 4.4922 & 8.83 & 0.177 & -11.96 & -22.09 & -12.91 \\
\hline $1440+122$ & 0.162 & BLL & -10.74501 & 8.44 & 3.8022 & 8.14 & 0.172 & -10.98 & -21.11 & -11.93 \\
\hline $1534+014$ & 0.312 & BLL & -11.10501 & 8.80 & 4.1622 & 8.50 & 0.335 & -12.31 & -22.44 & -13.26 \\
\hline $1704+604$ & 0.280 & BLL & -11.07501 & 8.77 & 4.1322 & 8.47 & 0.301 & -12.14 & -22.27 & -13.09 \\
\hline $1728+502$ & 0.055 & BLL & -10.43501 & 8.13 & 3.4922 & 7.83 & 0.060 & -9.40 & -19.53 & -10.35 \\
\hline $1757+703$ & 0.407 & BLL & -11.05501 & 8.75 & 4.1122 & 8.45 & 0.427 & -12.52 & -22.65 & -13.47 \\
\hline $1807+698$ & 0.051 & BLL & -12.40501 & 10.10 & 5.4622 & 9.80 & 0.502 & -12.78 & -22.91 & -13.73 \\
\hline $1853+671$ & 0.212 & BLL & -10.53501 & 8.23 & 3.5922 & 7.93 & 0.218 & -10.89 & -21.02 & -11.84 \\
\hline $2005-489$ & 0.071 & BLL & -11.33501 & 9.03 & 4.3922 & 8.73 & 0.109 & -11.21 & -21.34 & -12.16 \\
\hline $2143+070$ & 0.237 & BLL & -10.76501 & 8.46 & 3.8222 & 8.16 & 0.247 & -11.41 & -21.54 & -12.36 \\
\hline $2200+420$ & 0.069 & BLL & -10.53501 & 8.23 & 3.5922 & 7.93 & 0.075 & -9.79 & -19.92 & -10.74 \\
\hline $2254+074$ & 0.190 & BLL & -10.92501 & 8.62 & 3.9822 & 8.32 & 0.205 & -11.46 & -21.59 & -12.41 \\
\hline $2326+174$ & 0.213 & BLL & -11.04501 & 8.74 & 4.1022 & 8.44 & 0.233 & -11.79 & -21.92 & -12.74 \\
\hline $2356-309$ & 0.165 & BLL & -10.90501 & 8.60 & 3.9622 & 8.30 & 0.179 & -11.28 & -21.41 & -12.23 \\
\hline $0230-027$ & 0.239 & RG & -10.27501 & 7.97 & 3.3322 & 7.67 & 0.242 & -10.56 & -20.70 & -11.52 \\
\hline $0307+169$ & 0.256 & RG & -10.96501 & 8.66 & 4.0222 & 8.36 & 0.272 & -11.85 & -21.98 & -12.80 \\
\hline
\end{tabular}


TABLe 5: Continued.

\begin{tabular}{|c|c|c|c|c|c|c|c|c|c|c|}
\hline Name & $z$ & Type & $\log \left(\frac{R_{d}}{r_{\mathrm{ov}}}\right)$ & $\log \left(\frac{M_{\mathrm{BH}}}{M_{\odot}}\right)$ & $\log \left(\frac{M_{\mathrm{BH}}^{\text {Seed }}}{M_{\odot}}\right)$ & $\log T_{\mathrm{BH}}$ & $z^{\text {Seed }}$ & $J^{q}$ & $J^{\mathrm{URCA}}$ & $J^{\pi}$ \\
\hline $0345+337$ & 0.244 & RG & -9.42501 & 7.12 & 2.4822 & 6.82 & 0.244 & -9.10 & -19.23 & -10.05 \\
\hline $0917+459$ & 0.174 & $\mathrm{RG}$ & -10.51501 & 8.21 & 3.5722 & 7.91 & 0.180 & -10.65 & -20.78 & -11.60 \\
\hline $0958+291$ & 0.185 & RG & -10.23501 & 7.93 & 3.2922 & 7.63 & 0.188 & -10.23 & -20.36 & -11.18 \\
\hline $1215-033$ & 0.184 & $\mathrm{RG}$ & -10.23501 & 7.93 & 3.2922 & 7.63 & 0.187 & -10.22 & -20.35 & -11.17 \\
\hline $1215+013$ & 0.118 & RG & -10.50501 & 8.20 & 3.5622 & 7.90 & 0.124 & -10.25 & -20.38 & -11.20 \\
\hline $1330+022$ & 0.215 & RG & -10.12501 & 7.82 & 3.1822 & 7.52 & 0.217 & -10.19 & -20.32 & -11.14 \\
\hline $1342-016$ & 0.167 & RG & -10.71501 & 8.41 & 3.7722 & 8.11 & 0.176 & -10.96 & -21.09 & -11.91 \\
\hline $2141+279$ & 0.215 & RG & -10.12501 & 7.82 & 3.1822 & 7.52 & 0.217 & -10.19 & -20.32 & -11.14 \\
\hline $0257+024$ & 0.115 & RQQ & -11.05501 & 8.75 & 4.1122 & 8.45 & 0.135 & -11.18 & -21.32 & -12.13 \\
\hline $1549+203$ & 0.250 & RQQ & -9.22501 & 6.92 & 2.2822 & 6.62 & 0.250 & -8.78 & -18.91 & -9.73 \\
\hline $2215-037$ & 0.241 & RQQ & -10.50501 & 8.20 & 3.5622 & 7.90 & 0.247 & -10.98 & -21.11 & -11.93 \\
\hline $2344+184$ & 0.138 & RQQ & -9.37501 & 7.07 & 2.4322 & 6.77 & 0.138 & -8.42 & -18.56 & -9.38 \\
\hline $0958+291$ & 0.185 & RG & -10.23501 & 7.93 & 3.2922 & 7.63 & 0.188 & -10.23 & -20.36 & -11.18 \\
\hline $1215-033$ & 0.184 & RG & -10.23501 & 7.93 & 3.2922 & 7.63 & 0.187 & -10.22 & -20.35 & -11.17 \\
\hline $1215+013$ & 0.118 & $\mathrm{RG}$ & -10.50501 & 8.20 & 3.5622 & 7.90 & 0.124 & -10.25 & -20.38 & -11.20 \\
\hline $1330+022$ & 0.215 & $\mathrm{RG}$ & -10.12501 & 7.82 & 3.1822 & 7.52 & 0.217 & -10.19 & -20.32 & -11.14 \\
\hline $1342-016$ & 0.167 & $\mathrm{RG}$ & -10.71501 & 8.41 & 3.7722 & 8.11 & 0.176 & -10.96 & -21.09 & -11.91 \\
\hline $2141+279$ & 0.215 & RG & -10.12501 & 7.82 & 3.1822 & 7.52 & 0.217 & -10.19 & -20.32 & -11.14 \\
\hline $0257+024$ & 0.115 & RQQ & -11.05501 & 8.75 & 4.1122 & 8.45 & 0.135 & -11.18 & -21.32 & -12.13 \\
\hline $1549+203$ & 0.250 & RQQ & -9.22501 & 6.92 & 2.2822 & 6.62 & 0.250 & -8.78 & -18.91 & -9.73 \\
\hline $2215-037$ & 0.241 & RQQ & -10.50501 & 8.20 & 3.5622 & 7.90 & 0.247 & -10.98 & -21.11 & -11.93 \\
\hline $2344+184$ & 0.138 & RQQ & -9.37501 & 7.07 & 2.4322 & 6.77 & 0.138 & -8.42 & -18.56 & -9.38 \\
\hline
\end{tabular}

differential form of gauge field $F=(1 / 2) F_{n m} \vartheta^{n} \wedge \vartheta^{m}$. We connect the structure group $G_{V}$, further, to the nonlinear realization of the Lie group $G_{D}$ of distortion of extended space $M_{6}\left(\rightarrow \widetilde{M}_{6}\right)\left(\right.$ E.1), underlying the $M_{4}$. This extension appears to be indispensable for such a realization. In using the $6 \mathrm{D}$ language, we will be able to make a necessary reduction to the conventional $4 \mathrm{D}$ space. The laws guiding this redaction are given in Appendix E. The nonlinear realization technique or the method of phenomenological Lagrangians [85-91] provides a way to determine the transformation properties of fields defined on the quotient space. In accordance, we treat the distortion group $G_{D}$ and its stationary subgroup $H=$ $S O(3)$, respectively, as the dynamical group and its algebraic subgroup. The fundamental field is distortion gauge field (a) and, thus, all the fundamental gravitational structures in fact-the metric as much as the coframes and connectionsacquire a distortion-gauge induced theoretical interpretation. We study the geometrical structure of the space of parameters in terms of Cartan's calculus of exterior forms and derive the Maurer-Cartan structure equations, where the distortion fields (a) are treated as the Goldstone fields.

A.3. A Rearrangement of Vacuum State. Addressing the rearrangement of vacuum state, in realization of the group $G_{V}$ we implement the abelian local group [74]

$$
U^{\mathrm{loc}}=U(1)_{Y} \times \bar{U}(1) \equiv U(1)_{Y} \times \operatorname{diag}[S U(2)],
$$

on the space $M_{6}$ (spanned by the coordinates $\eta$ ), with the group elements of $\exp \left[i(Y / 2) \theta_{Y}(\eta)\right]$ of $U(1)_{Y}$ and $\exp \left[i T^{3} \theta_{3}(\eta)\right]$ of $\bar{U}(1)$. This group leads to the renormalizable theory, because gauge invariance gives a conservation of charge, and it also ensures the cancelation of quantum corrections that would otherwise result in infinitely large amplitudes. This has two generators, the third component $T^{3}$ of isospin $\vec{T}$ related to the Pauli spin matrix $\vec{\tau} / 2$, and hypercharge $Y$ implying $Q^{d}=T^{3}+Y / 2$, where $Q^{d}$ is the distortion charge operator assigning the number -1 to particles, but +1 to antiparticles. The group (A.3) entails two neutral gauge bosons of $\bar{U}(1)$, or that coupled to $T^{3}$, and of $U(1)_{Y}$, or that coupled to the hypercharge $Y$. Spontaneous symmetry breaking can be achieved by introducing the neutral complex scalar Higgs field. Minimization of the vacuum energy fixes the nonvanishing vacuum expectation value (VEV), which spontaneously breaks the theory, leaving the $U(1)_{d}$ subgroup intact, that is, leaving one Goldstone boson. Consequently, the left Goldstone boson is gauged away from the scalar sector, but it essentially reappears in the gauge sector providing the longitudinally polarized spin state of one of gauge bosons which acquires mass through its coupling to Higgs scalar. Thus, the two neutral gauge bosons were mixed to form two physical orthogonal states of the massless component of distortion field, $(a)\left(M_{a}=0\right)$, which is responsible for gravitational interactions, and its massive component, $(\bar{a})\left(M_{\bar{a}} \neq 0\right)$, which is responsible for the ID-regime. Hence, a substantial change of the properties of the spacetime continuum besides the curvature may arise at huge energies. This theory is renormalizable, because gauge invariance gives conservation of charge and also ensures the 
cancelation of quantum corrections that would otherwise result in infinitely large amplitudes. Without careful thought we expect that in this framework the renormalizability of the theory will not be spoiled in curved space-time too, because the infinities arise from ultraviolet properties of Feynman integrals in momentum space which, in coordinate space, are short distance properties, and locally (over short distances) all the curved spaces look like maximally symmetric (flat) space.

A.4. Model Building: Field Equations. The field equations follow at once from the total gauge invariant Lagrangian in terms of Euler-Lagrange variations, respectively, on both curved and flat spaces. The Lagrangian of distortion gauge field (a) defined on the flat space is undegenerated Killing form on the Lie algebra of the group $U^{\text {loc }}$ in adjoint representation, which yields the equation of distortion field (F.1). We are interested in the case of a spherical-symmetric gravitational field $a_{0}(r)$ in presence of one-dimensional spacelike ID-field $\bar{a}$ (F.6). In the case at hand, one has the group of motions $S O(3)$ with $2 \mathrm{D}$ space-like orbits $S^{2}$ where the standard coordinates are $\tilde{\theta}$ and $\tilde{\varphi}$. The stationary subgroup of $S O(3)$ acts isotropically upon the tangent space at the point of sphere $S^{2}$ of radius $\widetilde{r}$. So, the bundle $p: V_{4} \rightarrow \widetilde{R}^{2}$ has the fiber $S^{2}=p^{-1}(\tilde{x}), \tilde{x} \in V_{4}$, with a trivial connection on it, where $\widetilde{R}^{2}$ is the quotient-space $V_{4} / S O(3)$. Considering the equilibrium configurations of degenerate barionic matter, we assume an absence of transversal stresses and the transference of masses in $V_{4}$

$$
T_{1}^{1}=T_{2}^{2}=T_{3}^{3}=-\widetilde{P}(\widetilde{r}), \quad T_{0}^{0}=-\widetilde{\rho}(\widetilde{r}),
$$

where $\widetilde{P}(\widetilde{r})$ and $\widetilde{\rho}(\widetilde{r})\left(\widetilde{r} \in \widetilde{R}^{3}\right)$ are taken to denote the internal pressure and macroscopic density of energy defined in proper frame of reference that is being used. The equations of gravitation $\left(a_{0}\right)$ and ID $(\bar{a})$ fields can be given in Feynman gauge [71] as

$$
\begin{aligned}
& \Delta a_{0}=\frac{1}{2}\left\{\tilde{g}_{00} \frac{\partial \widetilde{g}^{00}}{\partial a_{0}} \tilde{\rho}(\widetilde{r})\right. \\
& \left.-\left[\tilde{g}_{33} \frac{\partial \widetilde{g}^{33}}{\partial a_{0}}+\widetilde{g}_{11} \frac{\partial \widetilde{g}^{11}}{\partial a_{0}}+\widetilde{g}_{22} \frac{\partial \widetilde{g}^{22}}{\partial a_{0}}\right] \widetilde{P}(\widetilde{r})\right\}, \\
& \left(\Delta-\lambda_{a}^{-2}\right) \bar{a}=\frac{1}{2}\left\{\tilde{g}_{00} \frac{\partial \widetilde{g}^{00}}{\partial \bar{a}} \widetilde{\rho}(\widetilde{r})\right. \\
& \left.-\left[\widetilde{g}_{33} \frac{\partial \widetilde{g}^{33}}{\partial \bar{a}}+\widetilde{g}_{11} \frac{\partial \widetilde{g}^{11}}{\partial \bar{a}}+\widetilde{g}_{22} \frac{\partial \widetilde{g}^{22}}{\partial \bar{a}}\right] \widetilde{P}(\widetilde{r})\right\} \\
& \times \theta\left(\lambda_{a}-\tilde{n}^{-1 / 3}\right),
\end{aligned}
$$

where $\tilde{n}$ is the concentration of particles and $\lambda_{a}=\hbar / m_{a} c \simeq$ $0.4 \mathrm{fm}$ is the Compton lenghth of the ID-field (but substantial ID-effects occur far below it), and a diffeomorphism $\widetilde{r}(r)$ : $M_{4} \rightarrow V_{4}$ is given as $r=\widetilde{r}-R_{g} / 4$. A distortion of the basis $\tilde{e}$ in the ID regime, in turn, yields the transformations of Poincaré generators of translations. Given an explicit form of distorted basis vectors (F.7), it is straightforward to derive the laws of phase transition for individual particle found in the ID-region $\left(x_{0}=0, x \neq 0\right)$ of the space-time continuum $\tan \widetilde{\theta}_{3}=-x, \widetilde{\theta}_{1}=\widetilde{\theta}_{1}=0$. The Poincaré generators $P_{\mu}$ of translations are transformed as follows [71]:

$$
\begin{gathered}
\widetilde{E}=E, \quad \widetilde{P}_{1,2}=P_{1,2} \cos \widetilde{\theta}_{3}, \\
\widetilde{P}_{3}=P_{3}-\tan \widetilde{\theta}_{3} m c \\
\widetilde{m}=\mid\left(m-\tan \widetilde{\theta}_{3} \frac{P_{3}}{c}\right)^{2} \\
+\sin ^{2} \widetilde{\theta}_{3} \frac{P_{1}^{2}+P_{2}^{2}}{c^{2}}-\left.\tan ^{2} \widetilde{\theta}_{3} \frac{E^{2}}{c^{4}}\right|^{1 / 2},
\end{gathered}
$$

where $E, \vec{P}$, and $m$ and $\widetilde{E}, \widetilde{\vec{P}}$, and $\widetilde{m}$ are ordinary and distorted energy, momentum, and mass at rest. Hence the matter found in the ID-region $(\bar{a} \neq 0)$ of space-time continuum has undergone phase transition of II-kind; that is, each particle goes off from the mass shell-a shift of mass and energymomentum spectra occurs upwards along the energy scale. The matter in this state is called protomatter with the thermodynamics differing strongly from the thermodynamics of ordinary compressed matter. The resulting deformed metric on $V_{4}$ in holonomic coordinate basis takes the form

$$
\begin{gathered}
\tilde{g}_{00}=\left(1-x_{0}\right)^{2}+x^{2}, \quad \tilde{g}_{\mu \nu}=0 \quad(\mu \neq \nu), \\
\tilde{g}_{33}=-\left[\left(1+x_{0}\right)^{2}+x^{2}\right], \quad \tilde{g}_{11}=-\widetilde{r}^{2}, \\
\tilde{g}_{22}=-\widetilde{r}^{2} \sin ^{2} \widetilde{\theta} .
\end{gathered}
$$

As a working model we assume the SPC-configurations given in Appendix G, which are composed of spherical-symmetric distribution of matter in many-phase stratified states. This is quick to estimate the main characteristics of the equilibrium degenerate barionic configurations and will guide us toward first look at some of the associated physics. The simulations confirm in brief the following scenario [71]: the energy density and internal pressure have sharply increased in protomatter core of SPC-configuration (with respect to corresponding central values of neutron star) proportional to gravitational forces of compression. This counteracts the collapse and equilibrium holds even for the masses $\sim 10^{9} M_{\odot}$. This feature can be seen, for example, from Figure 7 where the state equation of the II-class SPC $_{\text {II }}$ configuration, with the quark protomatter core, is plotted.

\section{B. A Hard Look at Spacetime Deformation}

The holonomic metric on $\widetilde{\mathscr{M}}_{4}$ can be recast in the form $\tilde{g}=\widetilde{g}_{\mu \nu} \widetilde{\vartheta}^{\mu} \otimes \widetilde{\vartheta}^{\nu}=\tilde{g}\left(\widetilde{e}_{\mu}, \widetilde{e}_{\nu}\right) \widetilde{\vartheta}^{\mu} \otimes \widetilde{\vartheta}^{\nu}$, with components $\widetilde{g}_{\mu \nu}=\widetilde{g}\left(\widetilde{e}_{\mu}, \widetilde{e}_{\nu}\right)$ in dual holonomic base $\left\{\widetilde{\vartheta}^{\mu} \equiv d \widetilde{x}^{\mu}\right\}$. In order to relate local Lorentz symmetry to more general deformed 


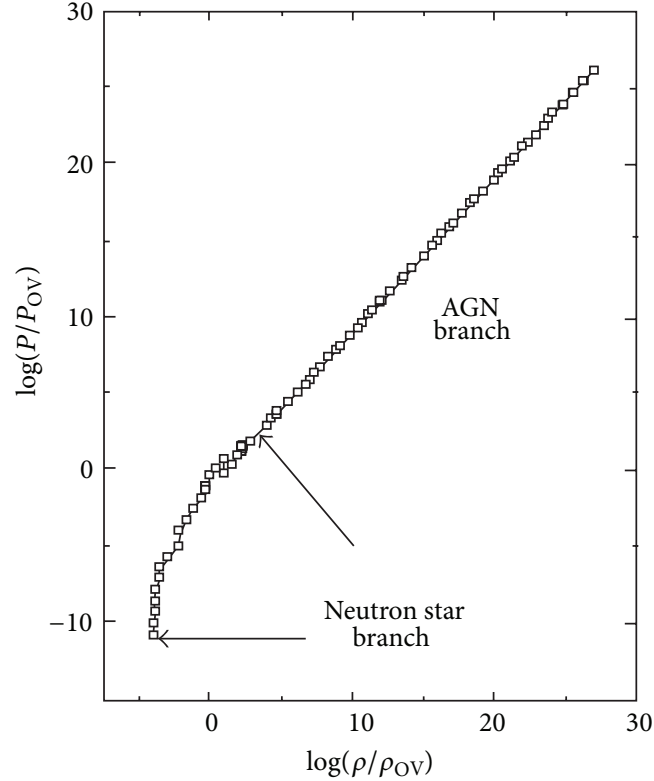

FIGURE 7: The state equation of $\mathrm{SPC}_{\mathrm{II}}$ on logarithmic scales, where $P$ and $\rho$ are the internal pressure and density, given in special units $P_{\mathrm{OV}}=6.469 \times 10^{36}\left[\mathrm{erg} \mathrm{cm}^{-3}\right]$ and $\rho_{\mathrm{OV}}=7.194 \times 10^{15}\left[\mathrm{~g} \mathrm{~cm}^{-3}\right]$, respectively.

spacetime, there is, however, a need to introduce the soldering tools, which are the linear frames and forms in tangent fiberbundles to the external smooth differential manifold, whose components are so-called tetrad (vierbein) fields. The $\widetilde{M}_{4}$ has at each point a tangent space, $\widetilde{T}_{\widetilde{x}} \widetilde{M}_{4}$, spanned by the anholonomic orthonormal frame field, $\widetilde{e}$, as a shorthand for the collection of the 4-tuplet $\left(\widetilde{e}_{0}, \ldots, \widetilde{e}_{3}\right)$, where $\widetilde{e}_{a}=\widetilde{e}_{a}^{\mu} \widetilde{\partial}_{\mu}$. We use the first half of Latin alphabet $(a, b, c, \ldots=0,1,2,3)$ to denote the anholonomic indices related to the tangent space. The frame field, $\widetilde{e}$, then defines a dual vector, $\widetilde{\vartheta}$, of differential forms, $\widetilde{\vartheta}=\left(\begin{array}{c}\widetilde{\vartheta}^{0} \\ \vdots \\ \tilde{\vartheta}^{3}\end{array}\right)$, as a shorthand for the collection of the $\widetilde{\vartheta}^{b}=\widetilde{e}_{\mu}^{b} d \widetilde{x}^{\mu}$, whose values at every point form the dual basis, such that $\left.\tilde{e}_{a}\right\rfloor \widetilde{\vartheta}^{b}=\delta_{a}^{b}$, where $\rfloor$ denotes the interior product; namely, this is a $C^{\infty}$-bilinear map $\rfloor: \Omega^{1} \rightarrow \Omega^{0}$ with $\Omega^{p}$ denoting the $C^{\infty}$-modulo of differential $p$-forms on $\widetilde{\mathscr{M}}_{4}$. In components, we have $\widetilde{e}_{a}^{\mu} \widetilde{e}_{\mu}^{b}=\delta_{a}^{b}$. On the manifold, $\widetilde{\mathscr{M}}_{4}$, the tautological tensor field, $\tilde{i d}$, of type $(1,1)$ can be defined which assigns to each tangent space the identity linear transformation. Thus, for any point $\tilde{x} \in \widetilde{\mathscr{M}}_{4}$ and any vector $\widetilde{\xi} \in \widetilde{T}_{\widetilde{x}} \widetilde{\mathscr{M}}_{4}$, one has $i \widetilde{d}(\widetilde{\xi})=\widetilde{\xi}$. In terms of the frame field, the $\widetilde{\vartheta}^{a}$ give the expression for $i \tilde{d}$ as $i \tilde{d}=\widetilde{e} \widetilde{\vartheta}=\widetilde{e}_{0} \otimes \widetilde{\vartheta}^{0}+\cdots \widetilde{e}_{3} \otimes \widetilde{\vartheta}^{3}$, in the sense that both sides yield $\widetilde{\xi}$ when applied to any tangent vector $\tilde{\xi}$ in the domain of definition of the frame field. One can also consider general transformations of the linear group, $G L(4, R)$, taking any base into any other set of four linearly independent fields. The notation, $\left\{\widetilde{e}_{a}, \widetilde{\vartheta}^{b}\right\}$, will be used below for general linear frames. Let us introduce so-called first deformation matrices, $\left(\pi(x)^{m}{ }_{k}\right.$ and $\left.\tilde{\pi}_{l}^{a}(\tilde{x})\right) \in G L(4, \widetilde{M})$ for all $\widetilde{x}$, as follows:

$$
\begin{gathered}
\widetilde{D}_{\mu}^{m}=\widetilde{e}_{\mu}{ }^{k} \pi^{m}{ }_{k}, \quad \widetilde{\psi}_{l}^{\mu}=\widetilde{e}^{\mu}{ }_{k} \pi^{k}{ }_{l}, \\
\widetilde{e}_{\mu}{ }^{k} \widetilde{e}^{\mu}{ }_{m}=\delta_{m}^{k}, \quad \widetilde{\pi}_{a}^{m}=\widetilde{e}_{a}{ }^{\mu} \widetilde{D}_{\mu}^{m}, \\
\widetilde{\pi}_{l}^{a}=\widetilde{e}_{\mu}^{a} \widetilde{\psi}_{l}^{\mu},
\end{gathered}
$$

where $\widetilde{g}_{\mu \nu} \widetilde{e}_{k}^{\mu} \widetilde{e}_{s}^{\nu}=\eta_{k s} ; \eta_{k s}$ is the metric on $M_{4}$. A deformation tensor, $\Omega^{m}{ }_{l}=\pi^{m}{ }_{k} \pi^{k}{ }_{l}$, yields local tetrad deformations

$$
\begin{array}{ll}
\tilde{e}_{a}=\tilde{\pi}_{a}^{m} e_{m}, & \tilde{\vartheta}^{a}=\tilde{\pi}_{l}^{a} \vartheta^{l}, \\
\bar{e}_{k}=\pi_{k}^{m} e_{m}, & \bar{\vartheta}^{k}=\pi^{k}{ }_{l} \vartheta^{l},
\end{array}
$$

and $i \widetilde{d}=\widetilde{e}_{a} \otimes \widetilde{\vartheta}^{a}=\bar{e}_{k} \otimes \bar{\vartheta}^{k} \in \widetilde{\mathscr{M}}_{4}$. The first deformation matrices $\pi$ and $\tilde{\pi}$, in general, give rise to the right cosets of the Lorentz group; that is, they are the elements of the quotient group $G L(4, \widetilde{M}) / S O(3,1)$. If we deform the cotetrad according to (B.2), we have two choices to recast metric as follows: either writing the deformation of the metric in the space of tetrads or deforming the tetrad field:

$$
\begin{aligned}
\tilde{g} & =o_{a b} \widetilde{\vartheta}^{a} \otimes \widetilde{\vartheta}^{b}=o_{a b} \tilde{\pi}^{a}{ }_{l} \tilde{\pi}_{m}^{b} \vartheta^{l} \otimes \vartheta^{m} \\
& =\gamma_{l m} \vartheta^{l} \otimes \vartheta^{m},
\end{aligned}
$$

where the second deformation matrix, $\gamma_{l m}$, reads $\gamma_{l m}=$ $o_{a b} \widetilde{\pi}_{l}^{a} \tilde{\pi}_{m}^{b}$. The deformed metric splits as

$$
\widetilde{g}_{\mu \nu}=\Upsilon^{2} \eta_{\mu \nu}+\widetilde{\gamma}_{\mu \nu}
$$

provided that $\Upsilon=\tilde{\pi}_{a}^{a}=\pi_{k}^{k}$ and

$$
\begin{aligned}
\tilde{\gamma}_{\mu \nu} & =\left(\gamma_{a l}-\Upsilon^{2} o_{a l}\right) \widetilde{e}_{\mu}^{a} \widetilde{e}_{v}^{l} \\
& =\left(\gamma_{k s}-\Upsilon^{2} \eta_{k s}\right) \widetilde{e}_{\mu}^{k} \widetilde{e}_{\nu}^{s} .
\end{aligned}
$$

The anholonomic orthonormal frame field, $\widetilde{e}$, relates $\widetilde{g}$ to the tangent space metric, $o_{a b}=\operatorname{diag}(+---)$, by $o_{a b}=$ $\widetilde{g}\left(\widetilde{e}_{a}, \widetilde{e}_{b}\right)=\widetilde{g}_{\mu \nu} \widetilde{e}_{a}^{\mu} \widetilde{e}_{b}{ }^{\nu}$, which has the converse $\widetilde{g}_{\mu \nu}=o_{a b} \widetilde{e}^{a}{ }_{\mu} \widetilde{e}^{b}{ }_{\nu}$ because $\widetilde{e}_{a}{ }^{\mu} \widetilde{e}^{a}{ }_{v}=\delta_{v}^{\mu}$. With this provision, we build up a worlddeformation tensor $\Omega$ yielding a deformation of the flat space $M_{4}$. The $\gamma_{l m}$ can be decomposed in terms of symmetric $\widetilde{\pi}_{(a l)}$ and antisymmetric $\tilde{\pi}_{[a l]}$ parts of the matrix $\tilde{\pi}_{a l}=o_{a c} \tilde{\pi}_{l}^{c}$ (or, resp., in terms of $\pi_{(k l)}$ and $\pi_{[k l]}$, where $\left.\pi_{k l}=\eta_{k s} \pi_{l}^{s}\right)$ as

$$
\begin{aligned}
\gamma_{a l}= & \widetilde{\Upsilon}^{2} o_{a l}+2 \widetilde{\Upsilon} \widetilde{\Theta}_{a l}+o_{c d} \widetilde{\Theta}_{a}^{c} \widetilde{\Theta}_{l}^{d} \\
& +o_{c d}\left(\widetilde{\Theta}^{c}{ }_{a} \widetilde{\varphi}^{d}{ }_{l}+\widetilde{\varphi}^{c}{ }_{a} \widetilde{\Theta}^{d}{ }_{l}\right)+o_{c d} \widetilde{\varphi}^{c}{ }_{a} \widetilde{\varphi}^{d}{ }_{l},
\end{aligned}
$$

where

$$
\tilde{\pi}_{a l}=\tilde{\Upsilon} o_{a l}+\widetilde{\Theta}_{a l}+\widetilde{\varphi}_{a l}
$$

$\widetilde{\Upsilon}=\tilde{\pi}_{a}^{a}, \widetilde{\Theta}_{a l}$ is the traceless symmetric part, and $\widetilde{\varphi}_{a l}$ is the skew symmetric part of the first deformation matrix. 
The anholonomy objects defined on the tangent space, $\widetilde{T}_{\widetilde{x}} \widetilde{M}_{4}$, read

$$
\widetilde{C}^{a}:=d \widetilde{\vartheta}^{a}=\frac{1}{2} \widetilde{C}^{a}{ }_{b c} \widetilde{\vartheta}^{b} \wedge \widetilde{\vartheta}^{c},
$$

where the anholonomy coefficients, $\widetilde{C}_{b c}^{a}$, which represent the curls of the base members, are

$$
\begin{aligned}
\widetilde{C}_{a b}^{c} & =-\widetilde{\vartheta}^{c}\left(\left[\widetilde{e}_{a}, \widetilde{e}_{b}\right]\right)=\widetilde{e}_{a}{ }^{\mu} \widetilde{e}_{b}{ }^{\nu}\left(\widetilde{\partial}_{\mu} \widetilde{e}_{\nu}^{c}-\widetilde{\partial}_{\nu} \widetilde{e}_{\mu}^{c}\right) \\
& =-\widetilde{e}_{\mu}^{c}\left[\widetilde{e}_{a}\left(\widetilde{e}_{b}{ }^{\mu}\right)-\widetilde{e}_{b}\left(\widetilde{e}_{a}{ }^{\mu}\right)\right] \\
& =2 \pi^{c}{ }_{l} \widetilde{e}_{m}{ }^{\mu}\left(\pi^{-1}{ }^{m} \widetilde{\partial}_{\mu} \pi^{-1}{ }_{b]}\right) .
\end{aligned}
$$

In particular case of constant metric in the tetradic space, the deformed connection can be written as

$$
\widetilde{\Gamma}_{b c}^{a}=\frac{1}{2}\left(\widetilde{C}_{b c}^{a}-o^{a a^{\prime}} o_{b b^{\prime}} \widetilde{C}_{a^{\prime} c}^{b^{\prime}}-o^{a a^{\prime}} o_{c c^{\prime}} \widetilde{C}_{a^{\prime} b}^{c^{\prime}}\right) .
$$

All magnitudes related to the $V_{4}$ will be denoted by an over “»”. According to (A.1), we have $\stackrel{\circ}{\Omega}_{l}^{m}=\stackrel{\circ}{D}_{\mu}^{m} \stackrel{\leftrightarrow}{\psi}_{l}^{\mu}$ and $\breve{\Omega}^{\mu}{ }_{v}=$ $\breve{D}_{\rho}^{\mu} \breve{\psi}_{\nu}^{\rho}$, provided

$$
\begin{array}{ll}
\stackrel{e}{e}_{\mu}=\stackrel{\circ}{D}_{\mu}^{l} e_{l}, & \stackrel{\circ}{e}_{\mu} \stackrel{\circ}{\psi}_{l}^{\mu}=\stackrel{\circ}{\Omega}_{l}^{m} e_{m}, \\
\tilde{e}_{\rho}=\breve{D}_{\rho}^{\mu} e_{\mu}, & \tilde{e}_{\rho} \breve{\psi}_{\nu}^{\rho}=\breve{\Omega}^{\mu}{ }_{\nu}{ }_{\nu}{ }_{\mu} .
\end{array}
$$

In analogy with (B.1), the following relations hold:

$$
\begin{aligned}
& \dot{D}_{\mu}^{m}=\dot{e}_{\mu}{ }_{\mu}{ }^{\pi^{m}}{ }_{k}, \quad \dot{\leftrightarrow}_{l}^{\mu}=\dot{e}^{\mu}{ }_{k} \stackrel{\circ}{\pi}_{l}^{k}, \\
& \dot{e}_{\mu}{ }^{k_{\circ} e^{\mu}}{ }_{m}=\delta_{m}^{k}, \quad \dot{\pi}_{a}{ }^{m}=\dot{e}_{a}{ }^{\mu} \stackrel{\circ}{D}_{\mu}^{m} \text {, } \\
& \stackrel{\circ}{\pi}_{l}^{a}=\dot{e}_{\mu}^{a}{ }_{\mu} \dot{\psi}_{l}^{\mu} \text {, }
\end{aligned}
$$

where $\stackrel{\circ}{\Omega}_{l}^{m}=\dot{\pi}_{\rho}^{m} \dot{\pi}^{\rho}{ }_{l}$ and $\breve{\Omega}^{\mu}{ }_{\nu}=\breve{\pi}^{\mu}{ }_{\rho} \breve{\pi}^{\rho}{ }_{v}$. We also have $\stackrel{\circ}{g}_{\mu \nu} \stackrel{\circ}{e}_{k} \mu_{e_{s}}{ }^{\nu}=\eta_{k s}$ and

$$
\begin{gathered}
\breve{D}_{\rho}^{\mu}=\breve{e}_{\nu}{ }_{\nu}^{\mu} \breve{\pi}^{\nu}, \quad \breve{\psi}_{\nu}^{\rho}=\breve{e}^{\rho}{ }_{\mu} \breve{\pi}^{\mu}{ }_{\nu}, \\
\breve{e}_{\nu}{ }^{\mu} \breve{e}_{\rho}^{\nu}=\delta_{\rho}^{\mu}, \quad \breve{\pi}_{a}^{\mu}=\breve{e}_{a}{ }^{\rho} \breve{D}_{\rho}^{\mu}, \\
\breve{\pi}^{a}{ }_{\nu}=\breve{e}^{a}{ }_{\rho} \breve{\psi}_{\nu}^{\rho} .
\end{gathered}
$$

The norm $d{ }^{\circ} \equiv i d$ of the displacement $d \dot{x}^{\mu}$ on $V_{4}$ can be written in terms of the spacetime structures of $M_{4}$ as

$$
\dot{i d}=\stackrel{\circ}{\dot{\vartheta}}=\dot{\Omega}_{l}^{m} e_{m} \otimes \vartheta^{l} \in V_{4} .
$$

The holonomic metric can be recast in the form

$$
\stackrel{\circ}{g}=\stackrel{g}{g}_{\mu \nu} \dot{\vartheta}^{\mu} \otimes \dot{\vartheta}^{\nu}=\stackrel{g}{g}\left(\dot{e}_{\mu}, \stackrel{e}{e}_{\nu}\right) \stackrel{\circ}{\vartheta}^{\mu} \otimes \dot{\vartheta}^{\nu} \text {. }
$$

The anholonomy objects defined on the tangent space, $\stackrel{\circ}{\dot{T}}_{\dot{x}} V_{4}$, read

$$
\dot{C}^{a}:=d \dot{\bullet}^{a}=\frac{1}{2} \stackrel{\circ}{C}_{b c}^{a} \stackrel{\circ}{\vartheta}^{b} \wedge \stackrel{\circ}{\vartheta}^{c},
$$

where the anholonomy coefficients, $\stackrel{\circ}{C}_{b c}$, which represent the curls of the base members, are

$$
\begin{aligned}
\dot{C}_{b c}^{c} & =-\dot{\vartheta}^{c}\left(\left[\dot{e}_{a}, \dot{e}_{b}\right]\right) \\
& =\dot{e}_{a}{ }_{a} e_{b}{ }^{\nu}\left(\dot{\partial}_{\mu} e^{c}{ }_{\nu}-\dot{\partial}_{\nu} \dot{e}^{c}{ }_{\mu}\right) \\
& =-\dot{e}^{c}{ }_{\mu}\left[\dot{e}_{a}\left(\dot{e}_{b}{ }^{\mu}\right)-\dot{e}_{b}\left(\dot{e}_{a}^{\mu}\right)\right] .
\end{aligned}
$$

The (anholonomic) Levi-Civita (or Christoffel) connection can be written as

$$
\left.\left.\left.\stackrel{\circ}{\Gamma}_{a b}:=\stackrel{\circ}{e}_{[a}\right\rfloor d \dot{\vartheta}_{b]}-\frac{1}{2}\left(\dot{e}_{a}\right\rfloor \dot{e}_{b}\right\rfloor d \dot{\vartheta}_{c}\right) \wedge \stackrel{\vartheta}{\vartheta}^{c},
$$

where $\dot{\vartheta}_{c}$ is understood as the down indexed 1-form $\dot{\vartheta}_{c}=$ $o_{c b} \vartheta^{\circ}$. The norm $\tilde{i d}$ (A.2) can then be written in terms of the spacetime structures of $V_{4}$ and $M_{4}$ as

$$
\begin{aligned}
\tilde{i d} & =\widetilde{\mathcal{\vartheta}} \widetilde{\mathcal{Q}}_{\rho} \widetilde{e}_{\rho} \otimes \widetilde{\mathcal{\vartheta}}^{\rho}=\widetilde{e}_{a} \otimes \widetilde{\mathcal{\vartheta}}^{a}=\breve{\Omega}^{\mu}{ }_{\nu}{ }_{\nu}{ }_{\mu} \otimes \dot{\vartheta}^{\nu} \\
& =\breve{\Omega}^{a}{ }_{b} \breve{e}_{a} \breve{\vartheta}^{b}=\Omega^{m}{ }_{l} e_{m} \otimes \mathcal{\vartheta}^{l} \in \widetilde{\mathscr{M}}_{4},
\end{aligned}
$$

provided

$$
\begin{aligned}
& \breve{\Omega}_{b}^{a}=\breve{\pi}^{a}{ }_{c} \breve{\pi}_{b}^{c}=\breve{\Omega}^{\mu}{ }_{\nu}{ }^{\circ}{ }_{\mu}^{a}{ }_{\mu} e_{b}{ }^{\nu}, \quad \tilde{e}_{\rho}=\breve{\pi}^{\nu}{ }_{\rho} \stackrel{e}{\nu}_{v}, \\
& \widetilde{\vartheta}^{\rho}=\breve{\pi}_{\mu}{ }^{\rho} \dot{\vartheta}^{\mu}, \quad \tilde{e}_{c}=\breve{\pi}_{c}{ }^{a}{ }^{\circ} e_{a}, \quad \widetilde{\vartheta}^{c}=\breve{\pi}_{b}^{c}{ }_{b}^{\circ b} .
\end{aligned}
$$

Under a local tetrad deformation (B.20), a general spin connection transforms according to

$$
\widetilde{\omega}_{b \mu}^{a}=\breve{\pi}_{c}{ }^{a} \stackrel{\circ}{\omega}_{d \mu}^{c} \tilde{\pi}_{b}^{d}+\breve{\pi}_{c}^{a} \widetilde{\partial}_{\mu} \breve{\pi}_{b}^{c}=\pi_{l}^{a} \widetilde{\partial}_{\mu} \pi_{b}^{l} .
$$

We have then two choices to recast metric as follows:

$$
\begin{aligned}
\tilde{\mathfrak{g}} & =o_{a b} \widetilde{\vartheta}^{a} \otimes \widetilde{\vartheta}^{b}=o_{a b} \breve{\pi}^{a} \breve{\pi}_{d}^{b} \dot{\vartheta}^{c} \otimes \dot{\vartheta}^{d} \\
& =\breve{\gamma}_{c d} \dot{\vartheta}^{c} \otimes \dot{\vartheta}^{d} .
\end{aligned}
$$

In the first case, the contribution of the Christoffel symbols constructed by the metric $\breve{\gamma}_{a b}=o_{c d} \breve{\pi}^{c} \breve{\pi}^{d}{ }_{b}$ reads

$$
\begin{aligned}
\widetilde{\Gamma}_{b c}^{a}= & \frac{1}{2}\left(\dot{C}_{b c}^{a}-\breve{\gamma}^{a a^{\prime}} \breve{\gamma}_{b b^{\prime}}{\stackrel{\circ}{b^{\prime}}}_{a^{\prime} c}-\breve{\gamma}^{a a^{\prime}} \breve{\gamma}_{c c^{\prime}}{\stackrel{\circ}{C^{C^{\prime}}}}_{a^{\prime} b}\right) \\
& \left.\left.\left.+\frac{1}{2} \breve{\gamma}^{a a l}\left(\dot{e}_{c}\right\rfloor d \breve{\gamma}_{b a^{\prime}}-\dot{e}_{b}\right\rfloor d \breve{\gamma}_{c a^{\prime}}-\dot{e}_{a^{\prime}}\right\rfloor d \breve{\gamma}_{b c}\right) .
\end{aligned}
$$

As before, the second deformation matrix, $\breve{\gamma}_{a b}$, can be decomposed in terms of symmetric, $\breve{\pi}_{(a b)}$, and antisymmetric, $\breve{\pi}_{[a b]}$, parts of the matrix $\breve{\pi}_{a b}=o_{a c} \breve{\pi}_{b}^{c}$. So,

$$
\breve{\pi}_{a b}=\breve{\Upsilon}_{a b}+\breve{\Theta}_{a b}+\breve{\varphi}_{a b} \text {, }
$$

where $\breve{\Upsilon}=\breve{\pi}_{a}^{a}, \breve{\Theta}_{a b}$ is the traceless symmetric part, and $\breve{\varphi}_{a b}$ is the skew symmetric part of the first deformation matrix. In analogy with (B.4), the deformed metric can then be split as

$$
\widetilde{g}_{\mu \nu}(\breve{\pi})=\breve{\Upsilon}^{2}(\breve{\pi}) \stackrel{\circ}{g}_{\mu \nu}+\breve{\gamma}_{\mu \nu}(\breve{\pi}),
$$


where

$$
\breve{\gamma}_{\mu \nu}(\breve{\pi})=\left[\breve{\gamma}_{a b}-\breve{\Upsilon}^{2} o_{a b}\right] e^{a}{ }_{\mu} e^{e b}{ }_{\nu} .
$$

The inverse deformed metric reads

$$
\tilde{g}^{\mu v}(\breve{\pi})=o^{c d} \breve{\pi}^{-1 a}{ }_{c} \breve{\pi}^{-1 b}{ }_{d} \dot{e}_{a}^{\mu} e_{b} e_{b}^{\nu},
$$

where $\breve{\pi}^{-1 a}{ }_{c} \breve{\pi}_{b}^{c}=\breve{\pi}_{b}^{c} \breve{\pi}^{-1 a}{ }_{c}=\delta_{b}^{a}$. The (anholonomic) LeviCivita (or Christoffel) connection is

$$
\left.\left.\left.\widetilde{\Gamma}_{a b}:=\widetilde{e}_{[a}\right\rfloor d \widetilde{\vartheta}_{b]}-\frac{1}{2}\left(\widetilde{e}_{a}\right\rfloor \widetilde{e}_{b}\right\rfloor d \widetilde{\vartheta}_{c}\right) \wedge \widetilde{\vartheta}^{c},
$$

where $\widetilde{\vartheta}_{c}$ is understood as the down indexed 1-form $\widetilde{\vartheta}_{c}=$ $o_{c b} \widetilde{\vartheta}^{b}$. Hence, the usual Levi-Civita connection is related to the original connection by the relation

$$
\widetilde{\Gamma}_{\rho \sigma}^{\mu}=\breve{\Gamma}_{\rho \sigma}^{\mu}+\breve{\Pi}_{\rho \sigma}^{\mu},
$$

provided

$$
\begin{aligned}
\Pi_{\rho \sigma}^{\mu}= & 2 \widetilde{g}^{\mu \nu} \breve{g}_{\nu(\rho)} \breve{\nabla}_{\sigma)} \breve{\Upsilon}-\breve{g}_{\rho \sigma} g^{\mu \nu} \breve{\nabla}_{\nu} \breve{\Upsilon} \\
& +\frac{1}{2} \widetilde{g}^{\mu \nu}\left(\breve{\nabla}_{\rho} \breve{\gamma}_{\nu \sigma}+\breve{\nabla}_{\sigma} \breve{\gamma}_{\rho \nu}-\breve{\nabla}_{\nu} \breve{\gamma}_{\rho \sigma}\right),
\end{aligned}
$$

where $\breve{\nabla}$ is the covariant derivative. The contravariant deformed metric, $\widetilde{g}^{\nu \rho}$, is defined as the inverse of $\widetilde{g}_{\mu \nu}$, such that $\widetilde{g}_{\mu \nu} \widetilde{g}^{\nu \rho}=\delta_{\mu}^{\rho}$. Hence, the connection deformation $\Pi_{\rho \sigma}^{\mu}$ acts like a force that deviates the test particles from the geodesic motion in the space, $V_{4}$. A metric-affine space $\left(\widetilde{M}_{4}, \widetilde{g}, \widetilde{\Gamma}\right)$ is defined to have a metric and a linear connection that need not be dependent on each other. In general, the lifting of the constraints of metric-compatibility and symmetry yields the new geometrical property of the spacetime, which are the nonmetricity 1-form $\widetilde{N}_{a b}$ and the affine torsion 2-form $\widetilde{T}^{a}$ representing a translational misfit (for a comprehensive discussion see [92-95]). These, together with the curvature 2form $\widetilde{R}_{a}^{b}$, symbolically can be presented as $[96,97]$

$$
\left(\widetilde{N}_{a b}, \widetilde{T}^{a}, \widetilde{R}_{a}^{b}\right) \sim \widetilde{D}\left(\widetilde{g}_{a b}, \widetilde{\vartheta}^{a}, \widetilde{\Gamma}_{a}^{b}\right),
$$

where $\widetilde{D}$ is the covariant exterior derivative. If the nonmetricity tensor $\widetilde{N}_{\lambda \mu \nu}=-\widetilde{\mathscr{D}}_{\lambda} \widetilde{g}_{\mu \nu} \equiv-\widetilde{g}_{\mu \nu ; \lambda}$ does not vanish, the general formula for the affine connection written in the spacetime components is

$$
\widetilde{\Gamma}_{\mu \nu}^{\rho}=\stackrel{\circ}{\Gamma}_{\mu \nu}^{\rho}+\widetilde{K}_{\mu \nu}^{\rho}-\widetilde{N}_{\mu \nu}^{\rho}+\frac{1}{2} \widetilde{N}_{(\mu \nu)}^{\rho},
$$

where $\stackrel{\circ}{\Gamma}_{\mu \nu}^{\rho}$ is the Riemann part and $\widetilde{K}_{\mu \nu}^{\rho}:=2 \widetilde{Q}_{(\mu \nu)}{ }^{\rho}+\widetilde{Q}_{\mu \nu}^{\rho}$ is the non-Riemann part, the affine contortion tensor. The torsion, $\widetilde{Q}_{\mu \nu}^{\rho}=(1 / 2) \widetilde{T}_{\mu \nu}^{\rho}=\widetilde{\Gamma}_{[\mu \nu]}^{\rho}$ given with respect to a holonomic frame, $d \widetilde{\vartheta}^{\rho}=0$, is the third-rank tensor, antisymmetric in the first two indices, with 24 independent components. In a presence of curvature and torsion, the coupling prescription of a general field carrying an arbitrary representation of the Lorentz group will be

$$
\widetilde{\partial}_{\mu} \longrightarrow \widetilde{D}_{\mu}=\widetilde{\partial}_{\mu}-\frac{i}{2}\left(\widetilde{\omega}_{\mu}^{a b}-\widetilde{K}_{\mu}^{a b}\right) J_{a b},
$$

with $J_{a b}$ denoting the corresponding Lorentz generator. The Riemann-Cartan manifold, $U_{4}$, is a particular case of the general metric-affine manifold $\widetilde{\mathscr{M}}_{4}$, restricted by the metricity condition $\widetilde{N}_{\lambda \mu \nu}=0$, when a nonsymmetric linear connection is said to be metric compatible. The Lorentz and diffeomorphism invariant scalar curvature, $\widetilde{R}$, becomes either a function of $\widetilde{e}_{\mu}^{a}$ only, or $\widetilde{g}_{\mu \nu}$ :

$$
\begin{aligned}
\widetilde{R}(\widetilde{\omega}) & \equiv \widetilde{e}_{a}{ }^{\mu} \widetilde{e}_{b}{ }^{\nu} \widetilde{R}_{\mu \nu}{ }^{a b}(\widetilde{\omega})=\widetilde{R}(\widetilde{g}, \widetilde{\Gamma}) \\
& \equiv \widetilde{g}^{\rho v} \widetilde{R}_{\rho \mu \nu}^{\mu}(\widetilde{\Gamma}) .
\end{aligned}
$$

\section{Determination of $\widetilde{D}$ and $\widetilde{\psi}$ in Standard Theory of Gravitation}

Let $\widetilde{\omega}^{a b}=\widetilde{\omega}_{\mu}^{a b} \wedge d \widetilde{x}^{\mu}$ be the 1-forms of corresponding connections assuming values in the Lorentz Lie algebra. The action for gravitational field can be written in the form

$$
\widetilde{S}_{g}=\stackrel{\circ}{S}+\widetilde{S}_{Q}
$$

where the integral

$$
\begin{aligned}
\stackrel{\circ}{S} & =-\frac{1}{4 æ} \int \star \stackrel{R}{R}=-\frac{1}{4 æ} \int \star \stackrel{R}{c d}_{c d} \wedge \widetilde{\vartheta}^{c} \wedge \widetilde{\vartheta}^{d} \\
& =-\frac{1}{2 æ} \int \stackrel{\circ}{R} \sqrt{-\widetilde{g}} d \Omega
\end{aligned}
$$

is the usual Einstein action, with the coupling constant relating to the Newton gravitational constant $æ=8 \pi G_{N} / c^{4}, S_{Q}$ is the phenomenological action of the spin-torsion interaction, and $\star$ denotes the Hodge dual. This is a $C^{\infty}$-linear map $\star$ : $\Omega^{p} \rightarrow \Omega^{n-p}$, which acts on the wedge product monomials of the basis 1-forms as $\star\left(\widetilde{\vartheta}^{a_{1} \cdots a_{p}}\right)=\varepsilon^{a_{1} \cdots a_{n}} \widetilde{e}_{a_{p+1} \cdots a_{n}}$. Here we used the abbreviated notations for the wedge product monomials, $\widetilde{\vartheta}^{a_{1} \cdots a_{p}}=\widetilde{\vartheta}^{a_{1}} \wedge \widetilde{\vartheta}^{a_{2}} \wedge \cdots \wedge \widetilde{\vartheta}^{a_{p}}$, defined on the $U_{4}$ space, the $\tilde{e}_{a_{i}}(i=p+1, \ldots, n)$ are understood as the down indexed 1-forms $\widetilde{e}_{a_{i}}=o_{a_{i}} \widetilde{b}^{b}$, and $\varepsilon^{a_{1} \cdots a_{n}}$ is the total antisymmetric pseudotensor. The variation of the connection 1-form $\widetilde{\omega}^{a b}$ yields

$$
\delta \widetilde{S}_{\mathrm{Q}}=\frac{1}{\mathfrak{x}} \int \star \widetilde{\mathscr{T}}_{a b} \wedge \delta \widetilde{\omega}^{a b},
$$

where

$$
\begin{aligned}
\star \widetilde{T}_{a b} & :=\frac{1}{2} \star\left(\widetilde{Q}_{a} \wedge \widetilde{e}_{b}\right)=\widetilde{Q}^{c} \wedge \widetilde{\vartheta}^{d} \varepsilon_{c d a b} \\
& =\frac{1}{2} \widetilde{Q}_{\mu \nu}^{c} \wedge \widetilde{e}^{d}{ }_{\alpha} \varepsilon_{a b c d} \widetilde{\vartheta}^{\mu \nu \alpha},
\end{aligned}
$$


and also

$$
\widetilde{Q}^{a}=\widetilde{D} \widetilde{\vartheta}^{a}=d \widetilde{\vartheta}^{a}+\widetilde{\omega}_{b}^{a} \wedge \widetilde{\vartheta}^{b}
$$

The variation of the action describing the macroscopic matter sources $\widetilde{S}_{m}$ with respect to the coframe $9^{a}$ and connection 1form $\widetilde{\omega}^{a b}$ reads

$$
\begin{aligned}
\delta \widetilde{S}_{m} & =\int \delta \widetilde{L}_{m} \\
& =\int\left(-\star \widetilde{\theta}_{a} \wedge \delta \widetilde{\vartheta}^{a}+\frac{1}{2} \star \widetilde{\Sigma}_{a b} \wedge \delta \widetilde{\omega}^{a b}\right),
\end{aligned}
$$

where $\star \widetilde{\theta}_{a}$ is the dual 3 -form relating to the canonical energymomentum tensor, $\widetilde{\theta}_{a}^{\mu}$, by

$$
\star \widetilde{\theta}_{a}=\frac{1}{3 !} \widetilde{\theta}_{a}^{\mu} \varepsilon_{\mu \nu \alpha \beta} \widetilde{\vartheta}^{\nu \alpha \beta}
$$

and $\star \widetilde{\Sigma}_{a b}=-\star \widetilde{\Sigma}_{b a}$ is the dual 3-form corresponding to the canonical spin tensor, which is identical with the dynamical spin tensor $\widetilde{S}_{a b c} ;$ namely,

$$
\star \widetilde{\Sigma}_{a b}=\widetilde{S}_{a b}^{\mu} \varepsilon_{\mu \nu \alpha \beta} \widetilde{\vartheta}^{\nu \alpha \beta} .
$$

The variation of the total action, $\widetilde{S}=\widetilde{S}_{g}+\widetilde{S}_{m}$, with respect to the $\widetilde{e}_{a}, \widetilde{\omega}^{a b}$ and $\widetilde{\Phi}$ gives the following field equations:

$$
\begin{aligned}
& \text { (1) } \frac{1}{2} \stackrel{\circ}{R}_{c d} \wedge \widetilde{\vartheta}^{c}=æ \widetilde{\theta}_{d}=0, \\
& \text { (2) } \star \widetilde{T}_{a b}=-\frac{1}{2} æ \star \widetilde{\Sigma}_{a b}, \\
& \text { (3) } \frac{\delta \widetilde{L}_{m}}{\delta \widetilde{\Phi}}=0, \quad \frac{\delta \widetilde{L}_{m}}{\delta \overline{\widetilde{\Phi}}}=0 .
\end{aligned}
$$

In the sequel, the DC-members $\widetilde{D}$ and $\widetilde{\psi}$ can readily be determined as follows:

$$
\widetilde{D}_{a}^{l}=\eta^{l m}\left\langle\widetilde{e}_{a}, e_{m}\right\rangle, \quad \widetilde{\psi}_{l}^{a}=\eta_{l m} \widetilde{\vartheta}^{a}\left(\vartheta^{-1}\right)^{m} .
$$

\section{The GGP in More Detail}

Note that an invariance of the Lagrangian $L_{\widetilde{\Phi}}$ under the infinite-parameter group of general covariance (A.5) in $V_{4}$ implies an invariance of $L_{\widetilde{\Phi}}$ under the $G_{V}$ group and vice versa if and only if the generalized local gauge transformations of the fields $\widetilde{\Phi}(\widetilde{x})$ and their covariant derivative $\widetilde{\nabla}_{\mu} \widetilde{\Phi}(\widetilde{x})$ are introduced by finite local $U_{V} \in G_{V}$ gauge transformations:

$$
\begin{gathered}
\widetilde{\Phi}^{\prime}(\widetilde{x})=U_{V}(\widetilde{x}) \widetilde{\Phi}(\widetilde{x}), \\
{\left[\widetilde{\gamma}^{\mu}(\widetilde{x}) \widetilde{\nabla}_{\mu} \widetilde{\Phi}(\widetilde{x})\right]^{\prime}=U_{V}(\widetilde{x})\left[\widetilde{\gamma}^{\mu}(\widetilde{x}) \widetilde{\nabla}_{\mu} \widetilde{\Phi}(\widetilde{x})\right] .}
\end{gathered}
$$

Here $\widetilde{\nabla}_{\mu}$ denotes the covariant derivative agreeing with the metric, $\widetilde{g}^{\mu \nu}=(1 / 2)\left(\widetilde{\gamma}^{\mu} \widetilde{\gamma}^{\nu}+\widetilde{\gamma}^{\gamma} \widetilde{\gamma}^{\mu}\right): \widetilde{\nabla}_{\mu}=\widetilde{\partial}_{\mu}+\widetilde{\Gamma}_{\mu}$, where $\widetilde{\Gamma}_{\mu}(\widetilde{x})=(1 / 2) J^{a b} \widetilde{e}_{a}^{\nu}(\widetilde{x}) \widetilde{\partial}_{\mu} \widetilde{e}_{b \nu}(\widetilde{x})$ is the connection and $J_{a b}$ are the generators of Lorentz group $\Lambda$. The tetrad components $\tilde{e}_{a}^{\mu}(\tilde{x})$ associate with the chosen representation $D(\Lambda)$ by which the $\widetilde{\Phi}(\widetilde{x})$ is transformed as $[D(\Lambda)]_{l \cdots k}^{l^{\prime} \ldots k^{\prime}} \widetilde{\Phi}(\widetilde{x})$, where $D(\Lambda)=I+(1 / 2) \widetilde{\omega}^{a b} J_{a b}, \widetilde{\omega}_{a b}=-\widetilde{\omega}_{b a}$ are the parameters of the Lorentz group. One has, for example, to set $\widetilde{\gamma}^{\mu}(\widetilde{x}) \rightarrow \widetilde{e}^{\mu}(\widetilde{x})$ for the fields of $\operatorname{spin}(j=0,1)$; for vector field $\left[J_{a b}\right]_{k}^{l}=$ $\delta_{a}^{l} \eta_{b k}-\delta_{b}^{l} \eta_{a k}$; but $\widetilde{\gamma}^{\mu}(\widetilde{x})=\widetilde{e}_{a}^{\mu}(\tilde{x}) \gamma^{a}$ and $J_{a b}=-(1 / 4)\left[\gamma_{a}, \gamma_{b}\right]$ for the spinor field $(j=1 / 2)$, where $\gamma^{a}$ are the Dirac matrices.

Given the principal fiber bundle $\widetilde{P}\left(V_{4}, G_{V} ; \widetilde{\mathcal{S}}\right)$ with the structure group $G_{V}$, the local coordinates $\widetilde{p} \in \widetilde{P}$ are $\widetilde{p}=$ $\left(\widetilde{x}, U_{V}\right)$, where $\tilde{x} \in V_{4}$ and $U_{V} \in G_{V}$, the total bundle space $\widetilde{P}$ is a smooth manifold, and the surjection $\widetilde{\mathcal{S}}$ is a smooth map $\widetilde{s}: \widetilde{P} \rightarrow V_{4}$. A set of open coverings $\left\{\widetilde{U}_{i}\right\}$ of $V_{4}$ with $\tilde{x} \in\left\{\widetilde{U}_{i}\right\} \subset V_{4}$ satisfy $\bigcup_{\alpha} \widetilde{u}_{\alpha}=V_{4}$. The collection of matter fields of arbitrary spins $\widetilde{\Phi}(\widetilde{x})$ take values in standard fiber over $\tilde{x}: \widetilde{s}^{-1}\left(\widetilde{U}_{i}\right)=\widetilde{U}_{i} \times \widetilde{F}_{\tilde{x}}$. The fibration is given as $\bigcup_{\tilde{x}} \widetilde{s}^{-1}(\tilde{x})=$ $\widetilde{P}$. The local gauge will be the diffeomorphism map $\tilde{\chi}_{i}$ : $\widetilde{U}_{i} \times_{V_{4}} G_{V} \rightarrow \widetilde{s}^{-1}\left(\widetilde{\mathscr{U}}_{i}\right) \in \widetilde{P}$, since $\widetilde{\chi}_{i}^{-1}$ maps $\widetilde{\mathcal{s}}^{-1}\left(\widetilde{\mathscr{U}}_{i}\right)$ onto the direct (Cartesian) product $\widetilde{\mathscr{U}}_{i} \times_{V_{4}} G_{V}$. Here $\times_{V_{4}}$ represents the fiber product of elements defined over space $V_{4}$ such that $\widetilde{s}\left(\tilde{\chi}_{i}\left(\tilde{x}, U_{V}\right)\right)=\tilde{x}$ and $\tilde{\chi}_{i}\left(\tilde{x}, U_{V}\right)=\tilde{\chi}_{i}\left(\tilde{x},(i d)_{G_{V}}\right) U_{V}=\tilde{\chi}_{i}(\tilde{x}) U_{V}$ for all $\tilde{x} \in\left\{\widetilde{u}_{i}\right\}$, where $(i d)_{G_{V}}$ is the identity element of the group $G_{V}$. The fiber $\widetilde{s}^{-1}$ at $\tilde{x} \in V_{4}$ is diffeomorphic to $\widetilde{F}$, where $\widetilde{F}$ is the fiber space, such that $\widetilde{s}^{-1}(\widetilde{x}) \equiv \widetilde{F}_{\widetilde{x}} \approx \widetilde{F}$. The action of the structure group $G_{V}$ on $\widetilde{P}$ defines an isomorphism of the Lie algebra $\tilde{\mathfrak{g}}$ of $G_{V}$ onto the Lie algebra of vertical vector fields on $\widetilde{P}$ tangent to the fiber at each $\widetilde{p} \in \widetilde{P}$ called fundamental. To involve a drastic revision of the role of gauge fields in the physical concept of the spacetime deformation, we generalize the standard gauge scheme by exploring a new special type of distortion gauge field, (a), which is assumed to act on the external spacetime groups. Then, we also consider the principle fiber bundle, $P\left(M_{4}, U^{\text {loc }} ; s\right)$, with the base space $M_{4}$, the structure group $U^{\text {loc }}$, and the surjection $s$. The matter fields $\Phi(x)$ take values in the standard fiber which is the Hilbert vector space where a linear representation $U(x)$ of group $U^{\text {loc }}$ is given. This space can be regarded as the Lie algebra of the group $U^{\text {loc }}$ upon which the Lie algebra acts according to the law of the adjoint representation: $a \leftrightarrow$ $\operatorname{ad} a \Phi \rightarrow[a, \Phi]$.

The GGP accounts for the gravitation gauge group $G_{V}$ generated by the hidden local internal symmetry $U^{\text {loc }}$. The physical system of the fields $\widetilde{\Phi}(\widetilde{x})$ defined on $V_{4}$ must be invariant under the finite local gauge transformations $U_{V}$ (D.1) of the Lie group of gravitation $G_{V}$ (see Scheme 1), where $R_{\psi}(a)$ is the matrix of unitary map:

$$
\begin{gathered}
R_{\psi}(a): \Phi \longrightarrow \widetilde{\Phi} \\
S(a) R_{\psi}(a):\left(\gamma^{k} D_{k} \Phi\right) \longrightarrow\left(\widetilde{\gamma}^{\nu}(\widetilde{x}) \nabla_{\nu} \widetilde{\Phi}\right) .
\end{gathered}
$$




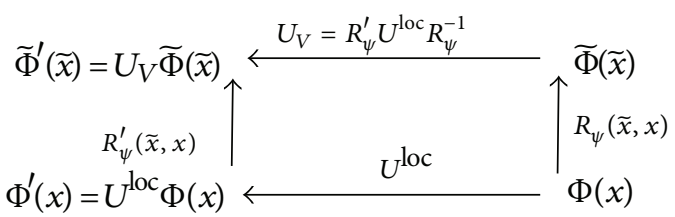

SCHEME 1: The GGP.

Here $S(F)$ is the gauge invariant scalar function $S(F) \equiv$ $(1 / 4) \widetilde{\pi}^{-1}(F)=(1 / 4) \widetilde{\psi}_{\mu}^{l} \widetilde{D}_{l}^{\mu}, D_{k}=\partial_{k}-i æ, a_{k}$. In an illustration of the point at issue, the (D.2) explicitly may read

$$
\begin{aligned}
\widetilde{\Phi}^{\mu \cdots \delta}(\widetilde{x}) & =\widetilde{\psi}_{l}^{\mu} \cdots \widetilde{\psi}_{m}^{\delta} R(a) \Phi^{l \cdots m}(x) \\
& \equiv\left(R_{\psi}\right)_{l \cdots m}^{\mu \cdots \delta} \Phi^{l \cdots m}(x),
\end{aligned}
$$

and also

$$
\begin{aligned}
& \widetilde{g}^{\nu}(\widetilde{x}) \nabla_{\nu} \widetilde{\Phi}^{\mu \cdots \delta}(\widetilde{x}) \\
& \quad=S(F) \widetilde{\psi}_{l}^{\mu} \cdots \widetilde{\psi}_{m}^{\delta} R(a) \gamma^{k} D_{k} \Phi^{l \cdots m}(x) .
\end{aligned}
$$

In case of zero curvature, one has $\psi_{l}^{\mu}=D_{l}^{\mu}=e^{\mu}{ }_{l}=$ $\left(\partial x^{\mu} / \partial X^{l}\right),\|D\| \neq 0$, where $X^{l}$ are the inertial coordinates. In this, the conventional gauge theory given on the $M_{4}$ is restored in both curvilinear and inertial coordinates. Although the distortion gauge field $\left(a_{A}\right)$ is a vector field, only the gravitational attraction is presented in the proposed theory of gravitation.

\section{E. A Lie Group of Distortion}

The extended space $M_{6}$ reads 0

$$
\begin{gathered}
M_{6}=R_{+}^{3} \oplus R_{-}^{3}=R^{3} \oplus T^{3}, \\
\operatorname{sgn}\left(R^{3}\right)=(+++), \quad \operatorname{sgn}\left(T^{3}\right)=(---) .
\end{gathered}
$$

The $e_{(\lambda \alpha)}=O_{\lambda} \times \sigma_{\alpha}(\lambda= \pm, \alpha=1,2,3)$ are linearly independent unit basis vectors at the point $(\mathrm{p})$ of interest of the given three-dimensional space $R_{\lambda}^{3}$. The unit vectors $O_{\lambda}$ and $\sigma_{\alpha}$ imply

$$
\left\langle O_{\lambda}, O_{\tau}\right\rangle={ }^{*} \delta_{\lambda \tau}, \quad\left\langle\sigma_{\alpha}, \sigma_{\beta}\right\rangle=\delta_{\alpha \beta},
$$

where $\delta_{\alpha \beta}$ is the Kronecker symbol and ${ }^{*} \delta_{\lambda \tau}=1-\delta_{\lambda \tau}$. Three spatial $e_{\alpha}=\xi \times \sigma_{\alpha}$ and three temporal $e_{0 \alpha}=\xi_{0} \times \sigma_{\alpha}$ components are the basis vectors, respectively, in spaces $R^{3}$ and $T^{3}$, where $O_{ \pm}=(1 / \sqrt{2})\left(\xi_{0} \pm \xi\right), \xi_{0}^{2}=-\xi^{2}=1,\left\langle\xi_{0}, \xi\right\rangle=0$. The 3D space $R_{ \pm}^{3}$ is spanned by the coordinates $\eta_{( \pm \alpha)}$. In using this language it is important to consider a reduction to the space $M_{4}$ which can be achieved in the following way.

(1) In case of free flat space $M_{6}$, the subspace $T^{3}$ is isotropic. And in so far it contributes in line element just only by the square of the moduli $t=\left|\mathbf{x}^{0}\right|, \mathbf{x}^{0} \in T^{3}$, then, the reduction $M_{6} \rightarrow M_{4}=R^{3} \oplus T^{1}$ can be readily achieved if we use $t=\left|\mathbf{x}^{0}\right|$ for conventional time.
(2) In case of curved space, the reduction $V_{6} \rightarrow V_{4}$ can be achieved if we use the projection $\left(\breve{e}_{0}\right)$ of the temporal component $\left(\breve{e}_{0 \alpha}\right)$ of basis six-vector $\breve{e}\left(\breve{e}_{\alpha}, \breve{e}_{0 \alpha}\right)$ on the given universal direction $\left(\breve{e}_{0 \alpha} \rightarrow \breve{e}_{0}\right)$. By this we choose the time coordinate. Actually, the Lagrangian of physical fields defined on $R_{6}$ is a function of scalars such that $A_{(\lambda \alpha)} B^{(\lambda \alpha)}=A_{\alpha} B^{\alpha}+A_{0 \alpha} B^{0 \alpha}$; then upon the reduction of temporal components of six-vectors $A_{0 \alpha} B^{0 \alpha}=A^{0 \alpha}\left\langle\breve{e}_{0 \alpha}, \breve{e}_{0 \beta}\right\rangle B^{0 \beta}=A^{0}\left\langle\breve{e}_{0}, \breve{e}_{0}\right\rangle B^{0}=A_{0} B^{0}$ we may fulfill a reduction to $V_{4}$.

A distortion of the basis (E.2) comprises the following two steps. We, at first, consider distortion transformations of the ingredient unit vectors $O_{\tau}$ under the distortion gauge field (a):

$$
\begin{aligned}
& \breve{O}_{(+\alpha)}(a)=Q_{(+\alpha)}^{\tau}(a) O_{\tau}=O_{+}+æ a_{(+\alpha)} O_{-}, \\
& \breve{O}_{(-\alpha)}(a)=\mathscr{Q}_{(-\alpha)}^{\tau}(a) O_{\tau}=O_{-}+æ a_{(-\alpha)} O_{+},
\end{aligned}
$$

where $\mathbb{Q}\left(=\mathbb{Q}_{(\lambda \alpha)}^{\tau}(a)\right)$ is an element of the group $Q$. This induces the distortion transformations of the ingredient unit vectors $\sigma_{\beta}$, which, in turn, undergo the rotations, $\breve{\sigma}_{(\lambda \alpha)}(\theta)=$ $\mathscr{R}_{(\lambda \alpha)}^{\beta}(\theta) \sigma_{\beta}$, where $\mathscr{R}(\theta) \in S O(3)$ is the element of the group of rotations of planes involving two arbitrary axes around the orthogonal third axis in the given ingredient space $R_{\lambda}^{3}$. In fact, distortion transformations of basis vectors $(O)$ and $(\sigma)$ are not independent but rather are governed by the spontaneous breaking of the distortion symmetry (for more details see [74]). To avoid a further proliferation of indices, hereafter we will use uppercase Latin $(A)$ in indexing $(\lambda \alpha)$, and so forth. The infinitesimal transformations then read

$$
\begin{gathered}
\delta \mathscr{Q}_{A}^{\tau}(a)=æ \delta a_{A} X_{\lambda}^{\tau} \in Q, \\
\delta \mathscr{R}(\theta)=-\frac{i}{2} M_{\alpha \beta} \delta \omega^{\alpha \beta} \in S O(3),
\end{gathered}
$$

provided by the generators $X_{\lambda}^{\tau}={ }^{*} \delta_{\lambda}^{\tau}$ and $I_{i}=\sigma_{i} / 2$, where $\sigma_{i}$ are the Pauli matrices, $M_{\alpha \beta}=\varepsilon_{\alpha \beta \gamma} I_{\gamma}$, and $\delta \omega^{\alpha \beta}=\varepsilon_{\alpha \beta \gamma} \delta \theta_{\gamma}$. The transformation matrix $D(a, \theta)=\mathbb{Q}(a) \times \mathscr{R}(\theta)$ is an element of the distortion group $G_{D}=Q \times S O(3)$ :

$$
\begin{gathered}
D_{\left(d a^{A}, d \theta^{A}\right)}=I+d D_{\left(a^{A}, \theta^{A}\right)} \\
d D_{\left(a^{A}, \theta^{A}\right)}=i\left[d a^{A} X_{A}+d \theta^{A} I_{A}\right],
\end{gathered}
$$

where $I_{A} \equiv I_{\alpha}$ at given $\lambda$. The generators $X_{A}$ (E.4) of the group $Q$ do not complete the group $H$ to the dynamical group $G_{D}$, and therefore they cannot be interpreted as the generators of the quotien space $G_{D} / H$, and the distortion fields $a_{A}$ cannot be identified directly with the Goldstone fields arising in spontaneous breaking of the distortion symmetry $G_{D}$. These objections, however, can be circumvented, because, as it is shown by [74], the distortion group $G_{D}=Q \times$ $S O$ (3) can be mapped in a one-to-one manner onto the group $G_{D}=S O(3) \times S O(3)$, which is isomorphic to the chiral group $S U(2) \times S U(2)$, in case of which the method of phenomenological Lagrangians is well known. In aftermath, we arrive at the key relation

$$
\tan \theta_{A}=-æ a_{A} .
$$


Given the distortion field $a_{A}$, the relation (E.6) uniquely determines six angles $\theta_{A}$ of rotations around each of six $(A)$ axes. In pursuing our goal further, we are necessarily led to extending a whole framework of GGP now for the base 12D smooth differentiable manifold:

$$
M_{12}=M_{6} \oplus \bar{M}_{6} .
$$

Here the $M_{6}$ is related to the spacetime continuum (E.1), but the $\bar{M}_{6}$ is displayed as a space of inner degrees of freedom. The

$$
e_{(\lambda, \mu, \alpha)}=O_{\lambda, \mu} \otimes \sigma_{\alpha} \quad(\lambda, \mu=1,2 ; \alpha=1,2,3)
$$

are basis vectors at the point $p(\zeta)$ of $M_{12}$ :

$$
\begin{aligned}
& \left\langle O_{\lambda, \mu}, O_{\tau, \nu}\right\rangle={ }^{*} \delta_{\lambda, \tau}{ }^{*} \delta_{\mu, \nu}, \quad O_{\lambda, \mu}=O_{\lambda} \otimes O_{\mu}, \\
& O_{\lambda, \mu} \longleftrightarrow{ }^{*} R^{4}={ }^{*} R^{2} \otimes^{*} R^{2}, \quad \sigma_{\alpha} \longleftrightarrow R^{3},
\end{aligned}
$$

where $\zeta=(\eta, u) \in M_{12}\left(\eta \in M_{6}\right.$ and $\left.u \in \bar{M}_{6}\right)$. So, the decomposition (E.1), together with

$$
\begin{gathered}
\bar{M}_{6}=\bar{R}_{+}^{3} \oplus \bar{R}_{-}^{3}=\bar{T}^{3} \oplus \bar{P}^{3}, \\
\operatorname{sgn}\left(\bar{T}^{3}\right)=(+++), \quad \operatorname{sgn}\left(\bar{P}^{3}\right)=(---),
\end{gathered}
$$

holds. The 12-dimensional basis (e) transforms under the distortion gauge field $a(\zeta)\left(\zeta \in M_{12}\right)$ :

$$
\widetilde{e}=D(a) e,
$$

where the distortion matrix $D(a)$ reads $D(a)=C(a) \otimes R(a)$, provided

$$
\widetilde{O}=C(a) O, \quad \widetilde{\sigma}=R(a) \sigma .
$$

The matrices $C(a)$ generate the group of distortion transformations of the bi-pseudo-vectors:

$$
C_{(\lambda \mu \alpha)}^{\tau, \nu}(a)=\delta_{\lambda}^{\tau} \delta_{\mu}^{\nu}+æ a_{(\lambda, \mu, \alpha)}{ }^{*} \delta_{\lambda}^{\tau *} \delta_{\mu}^{\nu},
$$

but $R(a) \in S O(3)_{\lambda \mu}$-the group of ordinary rotations of the planes involving two arbitrary bases of the spaces $R_{\lambda \mu}^{3}$ around the orthogonal third axes. The angles of rotations are determined according to (E.6), but now for the extended indices $A=(\lambda, \mu, \alpha)$ and so forth.

\section{F. Field Equations at Spherical Symmetry}

The extended field equations followed at once in terms of Euler-Lagrange variations, respectively, on the spaces $M_{12}$ and $\widetilde{M}_{12}$ [74]. In accordance, the equation of distortion gauge field $a_{A}=\left(a_{(\lambda \alpha)}, \bar{a}_{(\tau \beta)}\right)$ reads

$$
\begin{gathered}
\partial^{B} \partial_{B} a_{A}-\left(1-\zeta_{0}^{-1}\right) \partial_{A} \partial^{B} a_{B} \\
=J_{A}=-\frac{1}{2} \sqrt{g} \frac{\partial g^{B C}}{\partial a_{A}} T_{B C},
\end{gathered}
$$

where $T_{B C}$ is the energy-momentum tensor and $\zeta_{0}$ is the gauge fixing parameter. To render our discussion here more transparent, below we clarify the relation between gravitational and coupling constants. To assist in obtaining actual solutions from the field equations, we may consider the weakfield limit and will envisage that the right-hand side of (F.1) should be in the form

$$
-\frac{1}{2}\left(4 \pi G_{N}\right) \sqrt{g(x)} \frac{\partial g^{B C}(x)}{\partial x_{A}} \widetilde{T}_{B C} .
$$

Hence, we may assign to Newton's gravitational constant $G_{N}$ the value

$$
G_{N}=\frac{æ^{2}}{4 \pi}
$$

The curvature of manifold $M_{6} \rightarrow \widetilde{M_{6}}$ is the familiar distortion induced by the extended field components

$$
\begin{aligned}
& a_{(1,1, \alpha)}=a_{(2,1, \alpha)} \equiv \frac{1}{\sqrt{2}} a_{(+\alpha)}, \\
& a_{(1,2, \alpha)}=a_{(2,2, \alpha)} \equiv \frac{1}{\sqrt{2}} a_{(-\alpha)} .
\end{aligned}
$$

The other regime of ID presents at

$$
\begin{aligned}
& a_{(1,1, \alpha)}=-a_{(2,1, \alpha)} \equiv \frac{1}{\sqrt{2}} \bar{a}_{(+\alpha)}, \\
& a_{(1,2, \alpha)}=-a_{(2,2, \alpha)} \equiv \frac{1}{\sqrt{2}} \bar{a}_{(-\alpha)} .
\end{aligned}
$$

To obtain a feeling for this point we may consider physical systems which are static as well as spherically symmetrical. We are interested in the case of a spherical-symmetric gravitational field $a_{0}(r)$ in presence of one-dimensional space-like ID-field $\bar{a}$ :

$$
\begin{aligned}
& a_{(1,1,3)}=a_{(2,2,3)}=a_{(+3)}=\frac{1}{2}\left(-a_{0}+\bar{a}\right), \\
& a_{(1,2,3)}=a_{(2,1,3)}=a_{(-3)}=\frac{1}{2}\left(-a_{0}-\bar{a}\right), \\
& a_{(\lambda, \mu, 1)}=a_{(\lambda, \mu, 2)}=0, \quad \lambda, \mu=1,2 .
\end{aligned}
$$

One can then easily determine the basis vectors $\left(e_{\lambda \alpha}, \bar{e}_{\tau \beta}\right)$, where $\tan \theta_{( \pm 3)}=æ\left(-a_{0} \pm \bar{a}\right)$. Passing back from the $\widetilde{M}_{6}$ to $V_{4}$, the basis vectors read

$$
\begin{aligned}
& \tilde{e}_{0}=e_{0}\left(1-x_{0}\right)+\bar{e}_{3} x, \\
& \tilde{e}_{3}=e_{3}\left(1+x_{0}\right)-\bar{e}_{03} x,
\end{aligned}
$$




$$
\begin{gathered}
\tilde{e}_{1}=\frac{1}{2}\left\{\left(\cos \theta_{(+3)}+\cos \theta_{(-3)}\right) e_{1}\right. \\
+\left(\sin \theta_{(+3)}+\sin \theta_{(-3)}\right) e_{2} \\
+\left(\cos \theta_{(+3)}-\cos \theta_{(-3)}\right) \bar{e}_{01} \\
\left.+\left(\sin \theta_{(+3)}-\sin \theta_{(-3)}\right) \bar{e}_{02}\right\}, \\
\tilde{e}_{2}=\frac{1}{2}\left\{\left(\cos \theta_{(+3)}+\cos \theta_{(-3)}\right) e_{2}\right. \\
-\left(\sin \theta_{(+3)}+\sin \theta_{(-3)}\right) e_{1} \\
+\left(\cos \theta_{(+3)}-\cos \theta_{(-3)}\right) \bar{e}_{02} \\
\left.\quad-\left(\sin \theta_{(+3)}-\sin \theta_{(-3)}\right) \bar{e}_{01}\right\},
\end{gathered}
$$

where $x_{0} \equiv æ a_{0}, x \equiv æ \bar{a}$.

\section{G. SPC-Configurations}

The equations describing the equilibrium SPC include the gravitational and ID field equations (A.2), the hydrostatic equilibrium equation, and the state equation specified for each domain of many layered configurations. The resulting stable SPC is formed, which consists of the protomatter core and the outer layers of ordinary matter. A layering of configurations is a consequence of the onset of different regimes in equation of state. In the density range $\rho<$ $4.54 \times 10^{12} \mathrm{~g} \mathrm{~cm}^{-3}$, one uses for both configurations the simple semiempirical formula of state equation given by Harrison and Wheeler, see for example [98]. Above the density $\rho>4.54 \times 10^{12} \mathrm{~g} \mathrm{~cm}^{-3}$, for the simplicity, the Iclass SPC $_{\text {I }}$ configuration is thought to be composed of regular $n$-p-e (neutron-proton-electron) gas (in absence of ID) in intermediate density domain $4.54 \times 10^{12} \mathrm{~g} \mathrm{~cm}^{-3} \leq \rho<$ $\rho_{d}$ and of the n-p-e protomatter in presence of ID at $\rho>$ $\rho_{d}$. For the II-class $\mathrm{SPC}_{\mathrm{II}}$ configuration above the density $\rho_{f l}=4.09 \times 10^{14} \mathrm{~g} \mathrm{~cm}^{-3}$ one considers an onset of melting down of hadrons when nuclear matter consequently turns to quark matter, found in string flip-flop regime. In domain $\rho_{f l} \leq \rho<\rho_{d}$, to which the distances $0.4 \mathrm{fm}<r_{N N} \leq$ $1.6 \mathrm{fm}$ correspond, one has the regular (ID is absent) string flip-flop regime. This is a kind of tunneling effect when the strings joining the quarks stretch themselves violating energy conservation and after touching each other they switch on to the other configuration [71]. The basic technique adopted for calculation of transition matrix element $\widetilde{K}$ is the instanton technique (semiclassical treatment). During the quantum transition from a state $\psi_{1}$ of energy $\widetilde{E}_{1}$ to another one $\psi_{2}$ of energy $\widetilde{E}_{2}$, the lowering of energy of system takes place and the quark matter acquires $\Delta \widetilde{E}$ correction to the classical string energy such that the flip-flop energy lowers the energy of quark matter, consequently by lowering the critical density or critical Fermi momentum. If one, for example, looks for the string flip-flop transition amplitude of simple system of $q \bar{q} q \bar{q}$ described by the Hamiltonian $\widetilde{H}$ and invariant action $\widetilde{S}$, then one has

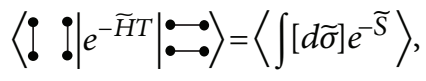

where $T$ is an imaginary time interval and $[d \tilde{\sigma}]$ is the integration over all the possible string motion. The action $\widetilde{S}$ is proportional to the area $\widetilde{A}$ of the surface swept by the strings in the finite region of ID-region of $R_{4}$. The strings are initially in the $:$-configuration and finally in the $!$-configuration. The maximal contribution to the path integral comes from the surface $\sigma_{0}$ of the minimum surface area "instanton". A computation of the transition amplitude is straightforward by summing over all the small vibrations around $\sigma_{0}$. In domain $\rho_{d} \leq \rho<\rho_{a s}$, one has the string flip-flop regime in presence of ID, at distances $0.25 \mathrm{fm}<r_{N N} \leq 0.4 \mathrm{fm}$. That is, the system is made of quark protomatter in complete $\beta$-equilibrium with rearrangement of string connections joining them. In final domain $\rho>\rho_{a s}$, the system is made of quarks in one bag in complete $\beta$-equilibrium at presence of ID. The quarks are under the weak interactions and gluons, including the effects of QCD-perturbative interactions. The QCD vacuum has a complicated structure, which is intimately connected to the gluon-gluon interaction. In most applications, sufficient accuracy is obtained by assuming that all the quarks are almost massless inside a bag. The latter is regarded as noninteracting Fermi gas found in the ID-region of the space-time continuum, at short distances $r_{N N} \leq 0.25 \mathrm{fm}$. Each configuration is defined by the two free parameters of central values of particle concentration $\widetilde{n}(0)$ and dimensionless potential of space-like ID-field $x(0)$. The interior gravitational potential $x_{0}^{\text {int }}(r)$ matches the exterior one $x_{0}^{\text {ext }}(r)$ at the surface of the configuration. The central value of the gravitational potential $x_{0}(0)$ can be found by reiterating integrations when the sewing condition of the interior and exterior potentials holds. The key question of stability of SPC was studied in [72]. In the relativistic case the total mass-energy of SPC is the extremum in equilibrium for all configurations with the same total number of baryons. While the extrema of $\widetilde{M}$ and $N$ occur at the same point in a one-parameter equilibrium sequence, one can look for the extremum of $\widetilde{E}=\widetilde{M} c^{2}-\widetilde{m}_{B} N$ on equal footing. Minimizing the energy will give the equilibrium configuration, and the second derivative of $\widetilde{E}$ will give stability information. Recall that, for spherical configurations of matter, instantaneously at rest, small radial deviations from equilibrium are governed by a Sturm-Liouville linear eigenvalue equation [98], with the imposition of suitable boundary conditions on normal modes with time dependence $\xi^{i}(\vec{x}, t)=\xi^{i}(\vec{x}) e^{i \omega t}$. A necessary and sufficient condition for stability is that the potential energy be positive defined for all initial data of $\xi^{i}(\vec{x}, 0)$, namely, in first order approximation when one does not take into account the rotation and magnetic field, if the square of frequency of normal mode of small perturbations is positive. A relativity tends to destabilize configurations. However, numerical integrations of the stability equations of SPC [72] give for the pressure-averaged value of the adiabatic index $\Gamma_{1}=(\partial \ln \widetilde{P} / \partial \ln \tilde{\rho})_{s}$ the following values: $\bar{\Gamma}_{1} \approx 2.216$ for 
the $\mathrm{SPC}_{\mathrm{I}}$ and $\bar{\Gamma}_{1} \approx 2.4$ for $\mathrm{SPC}_{\mathrm{II}}$ configurations. This clearly proves the stability of resulting SPC. Note that the SPC is always found inside the event horizon sphere, and therefore it could be observed only in presence of accreting matter.

\section{Conflict of Interests}

The author declares that there is no conflict of interests regarding the publication of this paper.

\section{Acknowledgment}

The very helpful and knowledgable comments from the anonymous referee which have essentially clarified the paper are much appreciated.

\section{References}

[1] J. A. Orosz, "Inventory of black hole binaries," in Proceedings of the International Astronomical Union Symposium (IAU '03), K. van der Hucht, A. Herrero, and C. Esteban, Eds., vol. 212, 2003.

[2] D. Lynden-Bell, "Galactic nuclei as collapsed old quasars," Nature, vol. 223, no. 5207, pp. 690-694, 1969.

[3] T. R. Lauer, S. M. Faber, D. Richstone et al., "The masses of nuclear black holes in luminous elliptical galaxies and implications for the space density of the most massive black holes," Astrophysical Journal Letters, vol. 662, no. 2 I, pp. 808834, 2007.

[4] M. Volonteri, "Formation of supermassive black holes," Astronomy and Astrophysics Review, vol. 18, no. 3, pp. 279-315, 2010.

[5] J. Woo and C. M. Urry, "Active galactic nucleus black hole masses and bolometric luminosities," Astrophysical Journal Letters, vol. 579, no. 2, pp. 530-544, 2002.

[6] E. Treister, P. Natarajan, D. B. Sanders, C. Megan Urry, K. Schawinski, and J. Kartaltepe, "Major galaxy mergers and the growth of supermassive black holes in quasars," Science, vol. 328, no. 5978, pp. 600-602, 2010.

[7] A. J. Davis and P. Natarajan, "Angular momentum and clustering properties of early dark matter haloes," Monthly Notices of the Royal Astronomical Society, vol. 393, no. 4, pp. 1498-1502, 2009.

[8] M. Vestergaard, "Early growth and efficient accretion of massive black holes at high redshift," Astrophysical Journal Letters, vol. 601, no. 2 I, pp. 676-691, 2004.

[9] M. Volonteri, G. Lodato, and P. Natarajan, "The evolution of massive black hole seeds," Monthly Notices of the Royal Astronomical Society, vol. 383, no. 3, pp. 1079-1088, 2008.

[10] M. Volonteri and P. Natarajan, "Journey to the MBH- $\sigma$ relation: the fate of low-mass black holes in the universe," Monthly Notices of the Royal Astronomical Society, vol. 400, no. 4, pp. 1911-1918, 2009.

[11] F. Shankar, D. H. Weinberg, and J. Miralda-Escudé, "Selfconsistent models of the AGN and black hole populations: duty cycles, accretion rates, and the mean radiative efficiency," Astrophysical Journal Letters, vol. 690, no. 1, pp. 20-41, 2009.

[12] B. C. Kelly, M. Vestergaard, X. Fan, P. Hopkins, L. Hernquist, and A. Siemiginowska, "Constraints on black hole growth, quasar lifetimes, and Eddington ratio distributions from the SDSS broad-line quasar black hole mass function," Astrophysical Journal Letters, vol. 719, no. 2, pp. 1315-1334, 2010.
[13] P. Natarajan, "The formation and evolution of massive black hole seeds in the early Universe," in Fluid Flows to Black Holes: A Tribute to S Chandrasekhar on His Birth Centenary, D. J. Saikia, Ed., pp. 191-206, World Scientific, 2011.

[14] E. Treister and C. M. Urry, "The cosmic history of black hole growth from deep multiwavelength surveys," Advances in Astronomy, vol. 2012, Article ID 516193, 21 pages, 2012.

[15] E. Treister, K. Schawinski, M. Volonteri, P. Natarajan, and E. Gawiser, "Black hole growth in the early Universe is selfregulated and largely hidden from view," Nature, vol. 474, no. 7351, pp. 356-358, 2011.

[16] C. J. Willott, L. Albert, D. Arzoumanian et al., "Eddingtonlimited accretion and the black hole mass function at redshift 6," Astronomical Journal, vol. 140, no. 2, pp. 546-560, 2010.

[17] V. Bromm and A. Loeb, "Formation of the first supermassive black holes," Astrophysical Journal Letters, vol. 596, no. 1, pp. 3446, 2003.

[18] B. Devecchi and M. Volonteri, "Formation of the first nuclear clusters and massive black holes at high redshift," Astrophysical Journal Letters, vol. 694, no. 1, pp. 302-313, 2009.

[19] A. J. Barger, L. L. Cowie, R. F. Mushotzky et al., "The cosmic evolution of hard X-ray-selected active galactic nuclei," Astronomical Journal, vol. 129, no. 2, pp. 578-609, 2005.

[20] S. M. Croom, G. T. Richards, T. Shanks et al., "The 2dF-SDSS LRG and QSO survey: the QSO luminosity function at $0.4<$ $z<2.6$," MNRAS, vol. 399, no. 4, pp. 1755-1772, 2009.

[21] Y. Ueda, M. Akiyama, K. Ohta, and T. Miyaji, "Cosmological evolution of the hard X-ray active galactic nucleus luminosity function and the origin of the hard X-ray background," The Astrophysical Journal Letters, vol. 598, no. 2 I, pp. 886-908, 2003.

[22] D. R. Ballantyne, A. R. Draper, K. K. Madsen, J. R. Rigby, and E. Treister, "Lifting the veil on obscured accretion: active galactic nuclei number counts and survey strategies for imaging hard X-ray missions," Astrophysical Journal, vol. 736, article 56, no. 1, 2011.

[23] T. Takahashi, K. Mitsuda, R. Kelley et al., "The ASTRO-H mission," in Space Telescopes and Instrumentation 2010: Ultraviolet to Gamma Ray, 77320Z, M. Arnaud, S. S. Murray, and T. Takahashi, Eds., vol. 7732 of Proceedings of SPIE, San Diego, Calif, USA, June 2010.

[24] L. Yao, E. R. Seaquist, N. Kuno, and L. Dunne, "CO molecular gas in infrared-luminous galaxies," Astrophysical Journal Letters, vol. 588, no. 2 I, pp. 771-791, 2003.

[25] A. Castellina and F. Donato, "Astrophysics of galactic charged cosmic rays," in Planets, Stars and Stellar Systems, T. D. Oswalt and G. Gilmore, Eds., vol. 5 of Galactic Structure and Stellar Populations, pp. 725-788, 2011.

[26] A. Letessier-Selvon and T. Stanev, "Ultrahigh energy cosmic rays," Reviews of Modern Physics, vol. 83, no. 3, pp. 907-942, 2011.

[27] G. Sigl, "High energy neutrinos and cosmic rays," http://arxiv .org/abs/1202.0466.

[28] K. Kotera, D. Allard, and A. V. Olinto, "Cosmogenic neutrinos: parameter space and detectabilty from $\mathrm{PeV}$ to $\mathrm{ZeV}$,' Journal of Cosmology and Astroparticle Physics, vol. 2010, article 013, 2010.

[29] D. Semikoz, "High-energy astroparticle physics," CERN Yellow Report CERN-2010-001, 2010.

[30] J. Linsley, "Primary cosmic-rays of energy 1017 to $1020 \mathrm{ev}$, the energy spectrum and arrival directions," in Proceedings of the 8th International Cosmic Ray Conference, vol. 4, p. 77, 1963.

[31] N. Hayashida, K. Honda, M. Honda et al., "Observation of a very energetic cosmic ray well beyond the predicted $2.7 \mathrm{~K}$ cutoff in 
the primary energy spectrum," Physical Review Letters, vol. 73, no. 26, pp. 3491-3494, 1994.

[32] D. J. Bird, S. C. Corbató, H. Y. Dai et al., "Detection of a cosmic ray with measured energy well beyond the expected spectral cutoff due to cosmic microwave radiation," Astrophysical Journal Letters, vol. 441, no. 1, pp. 144-150, 1995.

[33] K. Greisen, "End to the cosmic-ray spectrum?" Physical Review Letters, vol. 16, no. 17, pp. 748-750, 1966.

[34] G. T. Zatsepin and V. A. Kuz'min, "Upper limit of the spectrum of cosmic rays," Journal of Experimental and Theoretical Physics Letters, vol. 4, pp. 78-80, 1966.

[35] R. Abbasi, Y. Abdou, T. Abu-Zayyad et al., "Time-integrated searches for point-like sources of neutrinos with the 40-string IceCube detector," The Astrophysical Journal, vol. 732, no. 1, article 18, 2011.

[36] J. Abraham, "Observation of the suppression of the flux of cosmic rays above $4 £ 1019 \mathrm{eV}$," Physical Review Letters, vol. 101, no. 6, Article ID 061101, 2008.

[37] R. J. Protheroe and T. Stanev, "Limits on models of the ultrahigh energy cosmic rays based on topological defects," Physical Review Letters, vol. 77, no. 18, pp. 3708-3711, 1996.

[38] D. Fargion, "Ultrahigh energy neutrino scattering onto relic light neutrinos in galactic halo as a possible source of highest extragalactic cosmic rays," in Proceedings of the 25th International Cosmic Ray Conference (Held July-August, 1997 in Durban, South Africa), M. S. Potgieter, C. Raubenheimer, and D. J. van der Walt, Eds., vol. 7, pp. 153-156, Potchefstroom University, Transvaal, South Africa, 1997.

[39] D. Fargion, B. Mele, and A. Salis, "Ultra-high-energy neutrino scattering onto relic light neutrinos in the galactic halo as a possible source of the highest energy extragalactic cosmic rays," The Astrophysical Journal, vol. 517, no. 2, pp. 725-733, 1999.

[40] D. Fargion, B. Mele, and A. Salis, "Ultrahigh energy neutrino scattering onto relic light neutrinos in galactic halo as a possible source of highest energy extragalactic cosmic," Astrophysical Journal, vol. 517, pp. 725-733, 1999.

[41] T. J. Weiler, "Cosmic-ray neutrino annihilation on relic neutrinos revisited: a mechanism for generating air showers above the Greisen-Zatsepin-Kuzmin cutoff," Astroparticle Physics, vol. 11, no. 3, pp. 303-316, 1999.

[42] T. J. Weiler, "Cosmic-ray neutrino annihilation on relic neutrinos revisited: a mechanism for generating air showers above the Greisen-Zatsepin-Kuzmin cutoff,' Astroparticle Physics, vol. 11, no. 3, pp. 303-316, 1999.

[43] V. K. Dubrovich, D. Fargion, and M. Y. Khlopov, "Primordial bound systems of superheavy particles as the source of ultrahigh energy cosmic rays," Nuclear Physics B-Proceedings Supplements, vol. 136, no. 1-3, pp. 362-367, 2004.

[44] A. Datta, D. Fargion, and B. Mele, "Supersymmetry-neutrino unbound," Journal of High Energy Physics, 0509:007, 2005.

[45] S. Yoshida, G. Sigl, and S. Lee, "Extremely high energy neutrinos, neutrino hot dark matter, and the highest energy cosmic rays," Physical Review Letters, vol. 81, no. 25, pp. 5505-5508, 1998.

[46] A. Ringwald, "Possible detection of relic neutrinos and their mass," in Proceedings of the 27th International Cosmic Ray Conference, R. Schlickeiser, Ed., Invited, Rapporteur, and Highlight Papers, p. 262, Hamburg, Germany, August 2001.

[47] Z. Fodor, S. D. Katz, and A. Ringwald, "Relic neutrino masses and the highest energy cosmic-rays," Journal of High Energy Physics, 2002.
[48] O. E. Kalashev, V. A. Kuzmin, D. V. Semikoz, and G. Sigl, "Ultrahigh-energy neutrino fluxes and their constraints," Physical Review D, vol. 66, no. 6, Article ID 063004, 2002.

[49] O. E. Kalashev, V. A. Kuzmin, D. V. Semikoz, and G. Sigl, "Ultrahigh energy cosmic rays from neutrino emitting acceleration sources?" Physical Review D, vol. 65, no. 10, Article ID 103003, 2002.

[50] A. Y. Neronov and D. V. Semikoz, "Which blazars are neutrino loud?” Physical Review D, vol. 66, no. 12, Article ID 123003, 2002.

[51] P. Jain and S. Panda, "Ultra high energy cosmic rays from early decaying primordial black holes," in Proceedings of the 29th International Cosmic Ray Conference, B. Sripathi Acharya, Ed., vol. 9, pp. 33-36, Tata Institute of Fundamental Research, Pune, India, 2005.

[52] T. K. Gaisser, F. Halzen, and T. Stanev, "Particle astrophysics with high energy neutrinos," Physics Report, vol. 258, no. 3, pp. 173-236, 1995.

[53] J. Alvarez-Muñiz and F. Halzen, "Possible high-energy neutrinos from the cosmic accelerator RX J1713.7-3946," Astrophysical Journal Letters, vol. 576, no. 1, pp. L33-L36, 2002.

[54] P. S. Coppi and F. A. Aharonian, "Constraints on the very high energy emissivity of the universe from the diffuse $\mathrm{GeV}$ gammaray background," Astrophysical Journal Letters, vol. 487, no. 1, pp. L9-L12, 1997.

[55] D. Eichler, "High-energy neutrino astronomy-a probe of galactic nuclei," The Astrophysical Journal, vol. 232, pp. 106-112, 1979.

[56] F. Halzen and E. Zas, "Neutrino fluxes from active galaxies: a model-independent estimate," Astrophysical Journal Letters, vol. 488, no. 2, pp. 669-674, 1997.

[57] S. Adrian-Martinez, I. Al Samarai, A. Albert et al., "Measurement of atmospheric neutrino oscillations with the ANTARES neutrino tele-scope," Physics Letters B, vol. 714, no. 2-5, pp. 224230, 2012.

[58] S. Adrián-Martínez, J. A. Aguilar, I. Al Samarai et al., "First search for point sources of high energy cosmic neutrinos with the ANTARES neutrino telescope," The Astrophysical Journal Letters, vol. 743, no. 1, article L14, 2011.

[59] M. G. Aartsen, R. Abbasi, Y. Abdou et al., "Measurement of atmospheric neutrino oscillations with Ice-Cube," Physical Review Letters, vol. 111, no. 8, Article ID 081801, 2013.

[60] M. G. Aartsen, R. Abbasi, Y. Abdou et al., "First observation of PeV-energy neutrinos with IceCube," Physical Review Letters, vol. 111, Article ID 021103, 2013.

[61] M. G. Aartsen, "Evidence for high-energy extraterrestrial neutrinos at the IceCube detector," Science, vol. 342, no. 6161, Article ID 1242856, 2013.

[62] C. Kopper and IceCube Collabotration, "Observation of PeV neutrinos in IceCube," in Proceedings of the IceCube Particle Astrophysics Symposium (IPA '13), Madison, Wis, USA, May 2013, http://wipac.wisc.edu/meetings/home/IPA2013.

[63] N. Kurahashi-Neilson, "Spatial clustering analysis of the very high energy neutrinos in icecube," in Proceedings of the IceCube Particle Astrophysics Symposium (IPA '13), IceCube Collaboration, Madison, Wis, USA, May 2013.

[64] N. Whitehorn and IceCube Collabotration, "Results from IceCube," in Proceedings of the IceCube Particle Astrophysics Symposium (IPA '13), Madison, Wis, USA, May 2013.

[65] A. V. Avrorin, V. M. Aynutdinov, V. A. Balkanov et al., "Search for high-energy neutrinos in the Baikal neutrino experiment," Astronomy Letters, vol. 35, no. 10, pp. 651-662, 2009. 
[66] T. Abu-Zayyad, R. Aida, M. Allen et al., "The cosmic-ray energy spectrum observed with the surface detector of the tele-scope array experiment," The Astrophysical Journal Letters, vol. 768, no. 1, 5 pages, 2013.

[67] P. Abreu, M. Aglietta, M. Ahlers et al., "Large-scale distribution of arrival directions of cosmic rays detected above $10^{18} \mathrm{eV}$ at the pierre auger observatory," The Astrophysical Journal Supplement Series, vol. 203, no. 2, p. 20, 2012.

[68] "A search for point sources of EeV neutrons," The Astrophysical Journal, vol. 760, no. 2, Article ID 148, 11 pages, 2012.

[69] T. Ebisuzaki, Y. Takahashi, F. Kajino et al., "The JEM-EUSO mission to explore the extreme Universe," in Proceedings of the 7th Tours Symposium on Nuclear Physics and Astrophysics, vol. 1238, pp. 369-376, Kobe, Japan, November 2009.

[70] K. Murase, M. Ahlers, and B. C. Lacki, “Testing the hadronuclear origin of $\mathrm{PeV}$ neutrinos observed with IceCube," Physical Review D, vol. 88, Article ID 121301(R), 2013.

[71] G. Ter-Kazarian, "Protomatter and EHE C.R," Journal of the Physical Society of Japan B, vol. 70, pp. 84-98, 2001.

[72] G. Ter-Kazarian, S. Shidhani, and L. Sargsyan, "Neutrino radiation of the AGN black holes," Astrophysics and Space Science, vol. 310, no. 1-2, pp. 93-110, 2007.

[73] G. Ter-Kazarian and L. Sargsyan, "Signature of plausible accreting supermassive black holes in Mrk 261/262 and Mrk 266," Advances in Astronomy, vol. 2013, Article ID 710906, 12 pages, 2013.

[74] G. T. Ter-Kazarian, "Gravitation and inertia; a rearrangement of vacuum in gravity," Astrophysics and Space Science, vol. 327, no. 1, pp. 91-109, 2010.

[75] G. Ter-Kazarian, "Ultra-high energy neutrino fluxes from supermassive AGN black holes," Astrophysics \& Space Science, vol. 349, pp. 919-938, 2014.

[76] G. T. Ter-Kazarian, "Gravitation gauge group," Il Nuovo Cimento, vol. 112, no. 6, pp. 825-838, 1997.

[77] A. Neronov, D. Semikoz, F. Aharonian, and O. Kalashev, "Largescale extragalactic jets powered by very-high-energy gamma rays," Physical Review Letters, vol. 89, no. 5, pp. 1-4, 2002.

[78] T. Eguchi, P. B. Gilkey, and A. J. Hanson, "Gravitation, gauge theories and differential geometry," Physics Reports C, vol. 66, no. 6, pp. 213-393, 1980.

[79] S. Kobayashi and K. Nomizu, Foundations of Differential Geometry, Interscience Publishers, New York, NY, USA, 1963.

[80] J. Plebanski, "Forms and riemannian geometry," in Proceedings of the International School of Cosmology and Gravitation, Erice, Italy, 1972.

[81] A. Trautman, Differential Geometry for Physicists, vol. 2 of Monographs and Textbooks in Physical Science, Bibliopolis, Naples, Fla, USA, 1984.

[82] G. Ter-Kazarian, "Two-step spacetime deformation induced dynamical torsion," Classical and Quantum Gravity, vol. 28, no. 5, Article ID 055003, 2011.

[83] L. S. Pontryagin, Continous Groups, Nauka, Moscow, Russia, 1984.

[84] B. A. Dubrovin, Contemporary Geometry, Nauka, Moscow, Russia, 1986.

[85] S. Coleman, J. Wess, and B. Zumino, "Structure of phenomenological lagrangians. I," Physical Review, vol. 177, no. 5, pp. 22392247, 1969.

[86] C. G. Callan, S. Coleman, J. Wess, and B. Zumino, "Structure of phenomenological lagrangians. II," Physical Review, vol. 177, no. 5, pp. 2247-2250, 1969.
[87] S. Weinberg, Brandeis Lectures, MIT Press, Cambridge, Mass, USA, 1970 .

[88] A. Salam and J. Strathdee, "Nonlinear realizations: II. Conformal symmetry," Physical Review, vol. 184, pp. 1760-1768, 1969.

[89] C. J. Isham, A. Salam, and J. Strathdee, "Nonlinear realizations of space-time symmetries. Scalar and tensor gravity," Annals of Physics, vol. 62, pp. 98-119, 1971.

[90] D. V. Volkov, "Phenomenological lagrangians," Soviet Journal of Particles and Nuclei, vol. 4, pp. 1-17, 1973.

[91] V. I. Ogievetsky, "Nonlinear realizations of internal and spacetime symmetries," in Proceedings of 10th Winter School of Theoretical Physics in Karpacz, vol. 1, p. 117, Wroclaw, Poland, 1974.

[92] V. de Sabbata and M. Gasperini, "Introduction to gravitation," in Unified Field Theories of more than Four Dimensions, V. de Sabbata and E. Schmutzer, Eds., p. 152, World Scientific, Singapore, 1985.

[93] V. de Sabbata and M. Gasperini, "On the Maxwell equations in a Riemann-Cartan space," Physics Letters A, vol. 77, no. 5, pp. 300-302, 1980.

[94] V. de Sabbata and C. Sivaram, Spin and Torsion in Gravitation, World Scientific, Singapore, 1994.

[95] N. J. Poplawski, "Spacetime and fields," http://arxiv.org/abs/ 0911.0334.

[96] A. Trautman, "On the structure of the Einstein-Cartan equations," in Differential Geometry, vol. 12 of Symposia Mathematica, pp. 139-162, Academic Press, London, UK, 1973.

[97] J.-P. Francoise, G. L. Naber, and S. T. Tsou, Eds., Encyclopedia of Mathematical Physics, Elsevier, Oxford, UK, 2006.

[98] S. L. Shapiro and S. A. Teukolsky, Black Holes, White Dwarfs, and Neutron Stars, A Wiley-Intercience Publication, WileyIntercience, New York, NY, USA, 1983. 

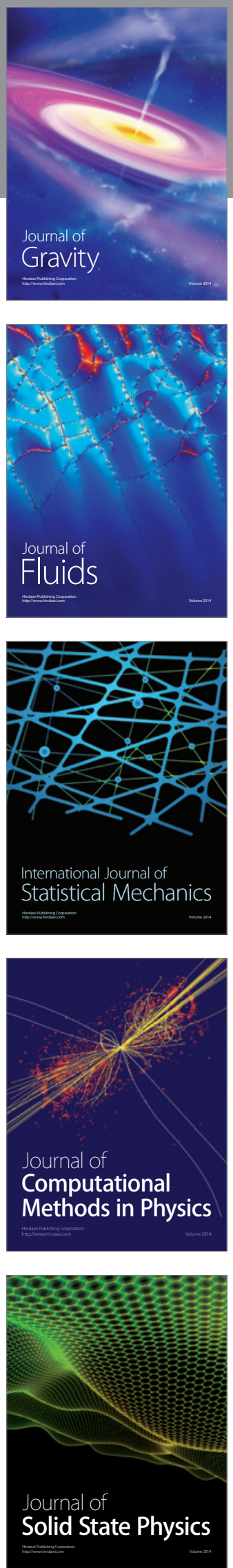

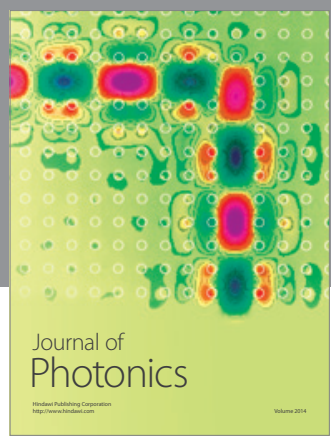

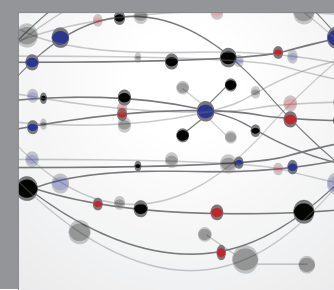

The Scientific World Journal

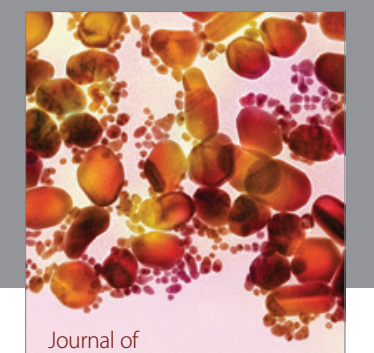

Soft Matter
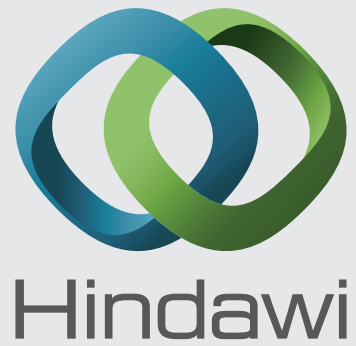

Submit your manuscripts at

http://www.hindawi.com
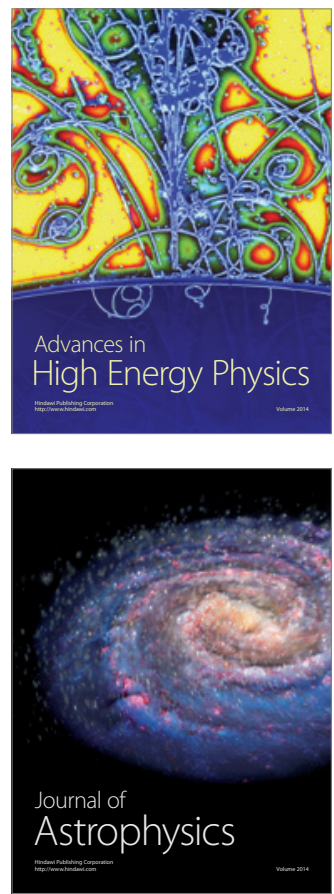
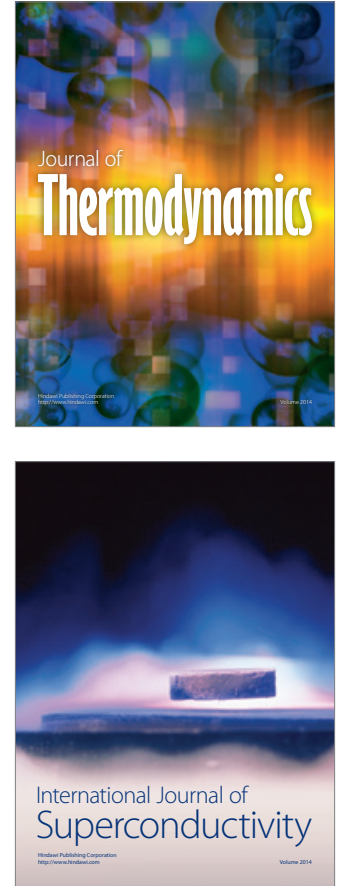
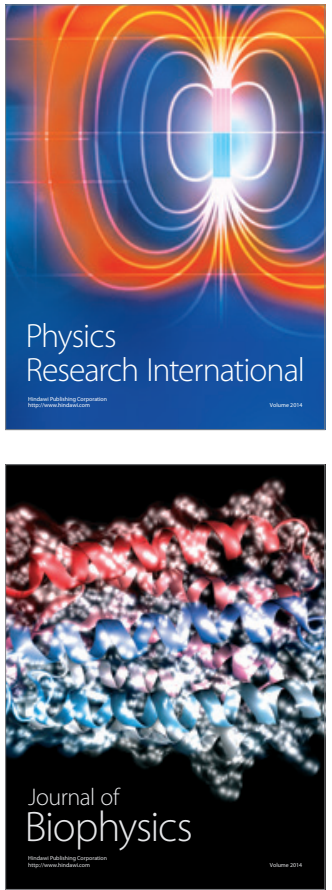
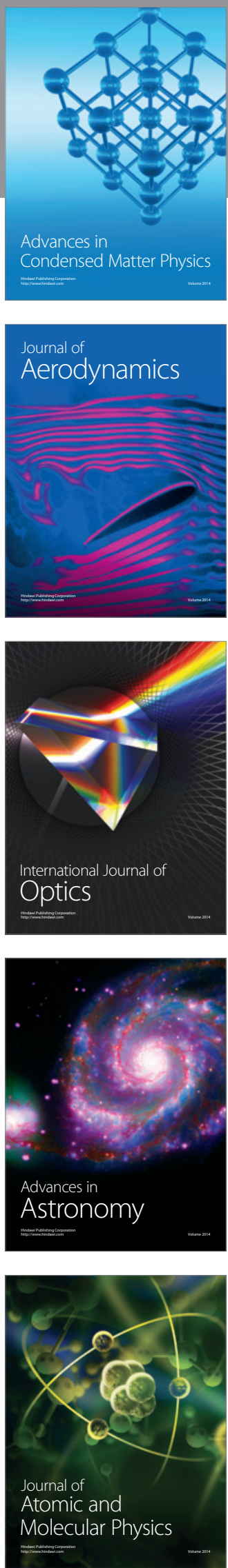